\title{
Visual Feedback to Perform Bend Gestures on Flexible Displays
}

\author{
by
}

Farshad Daliri

A thesis submitted to the Faculty of Graduate and Postdoctoral Affairs in partial fulfillment of the requirements for the degree of

\author{
Master of Applied Science \\ in \\ Human Computer Interaction
}

Carleton University

Ottawa, Ontario

(C) 2015

Farshad Daliri 


\section{Abstract}

Bend gestures offer a rich interaction language for flexible displays due to their large degrees of freedom. However, without an understandable visual feedback system, working with such interaction could be a difficult challenge for users. In this thesis, we have developed three visual feedback systems by using feedforward and feedback mechanisms with the goal of guiding users to correctly perform bend gestures on a handheld flexible display. Additionally, we have conducted an experiment containing two tasks, which, using a flexible prototype, evaluate the usefulness of our visual feedback designs. Our results suggested that users could perform sets of bend gestures faster when the visual feedbacks appear at the exact location of the bend gesture instead of always at a fixed location on the screen. Also, we found that, while feedforward improved users' performance, using feedback had a negative effect. Finally, we propose a set of design guidelines for designing visual feedback systems for bend gestures on flexible devices. 


\section{Acknowledgements}

It is difficult to overstate my gratitude to my supervisor, Dr. Audrey Girouard. I am thankful for her excellent guidance, kindness, and extraordinary patience with my mistakes. I am grateful to her for believing in me and never giving up on me even during my slow progress and difficulties. I thank her for understanding all my problems and dealing with them with cordial care. I thank her for spending countless hours of her valuable time to read my numerous drafts and providing me with valuable feedback. I could not have imagined having a better advisor and mentor for my thesis.

I want to thank my parents for being the best parents in the world and for always supporting me in every possible way. I consider myself extremely lucky to be their son and I wish someday I could return their kindness. I also want to thank my brother and sister, Farzad and Maryam, for helping me with their kind support and advice during my studies.

I would also like to share my appreciation for the staff of the School of Information Technology at Carleton University, especially Erenia Hernandez Oliver for her continuous support and help. 


\section{Table of Contents}

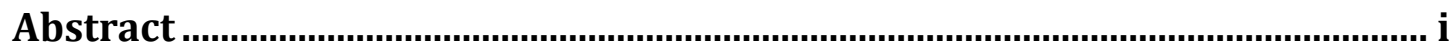

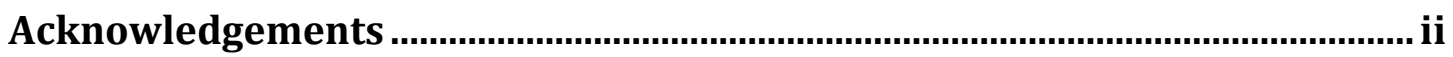

List of Figures ................................................................................................. vi

1.Introduction ...................................................................................................... 1

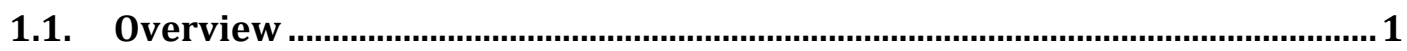

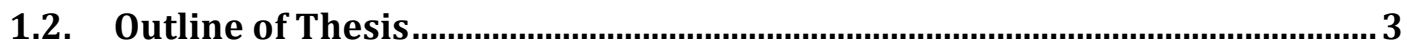

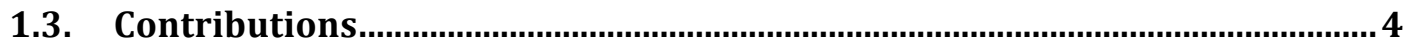

2.Related Works ……............................................................................. 5

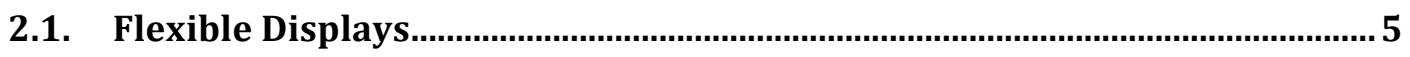

2.1.1. Physical Aspects of Flexible Displays ................................................................... 5

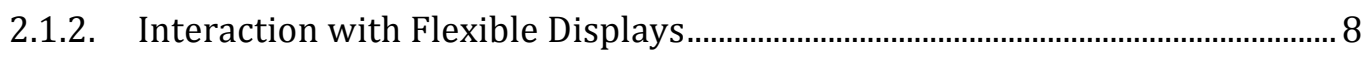

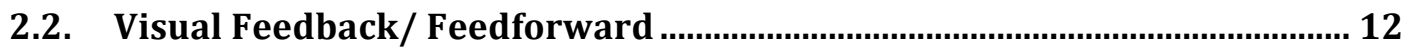

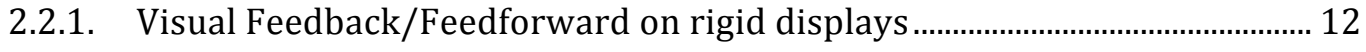

2.2.2. Visual Feeback/ Feedforward on Flexible displays .......................................... 16

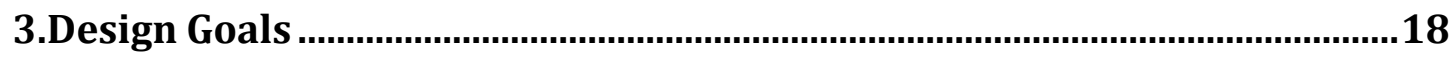

3.1. Potential problems during performing bend gestures ................................ 19

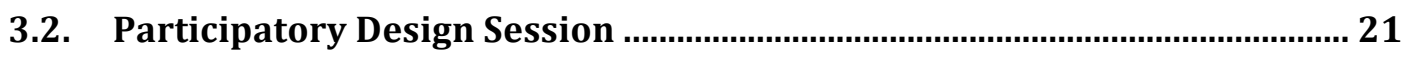

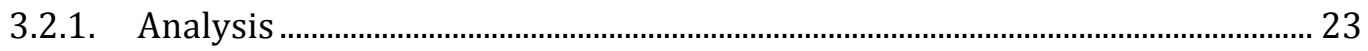

4.Designing Visual Feedbacks.............................................................................31

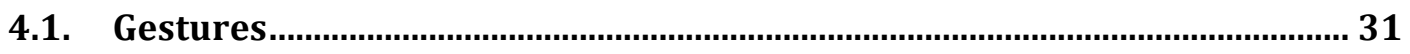

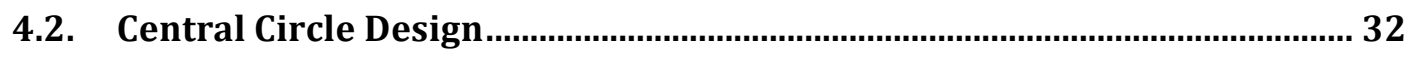

4.2.1. Feedforward Mechanism in the Central Circle Design ..................................... 34 
4.2.2. Feedback Mechanism in the Central Circle Design

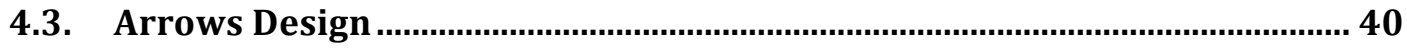

4.3.1. Feedback Mechanism in Arrows Design ................................................................. 45

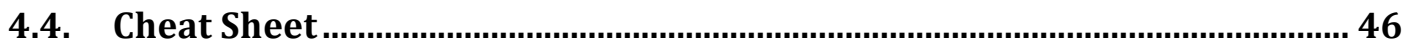

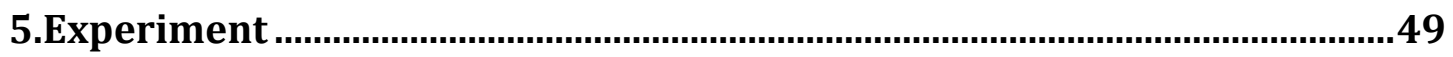

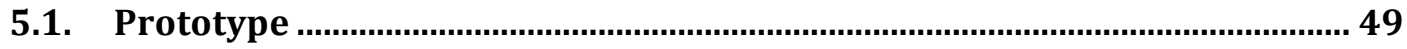

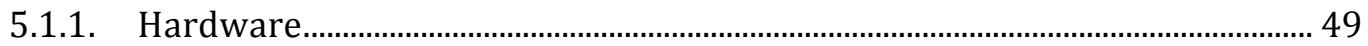

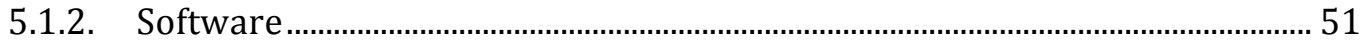

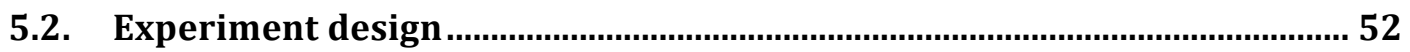

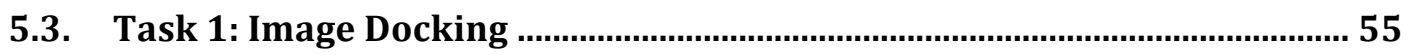

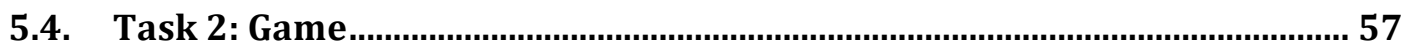

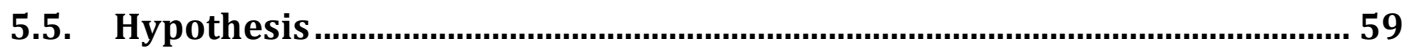

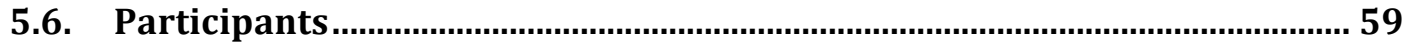

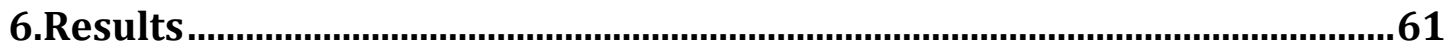

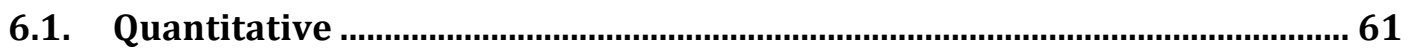

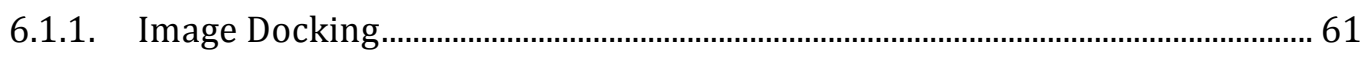

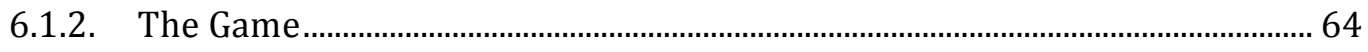

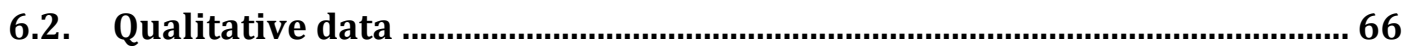

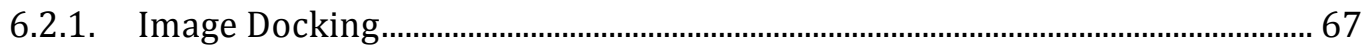

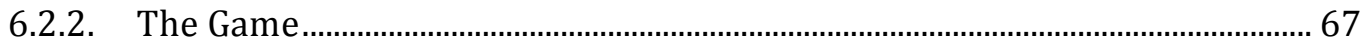

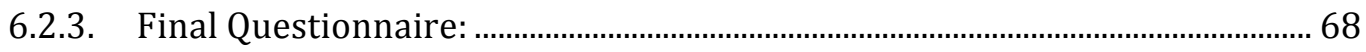

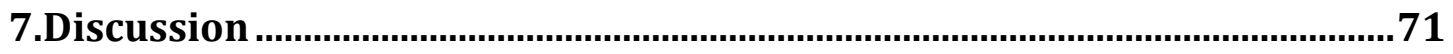

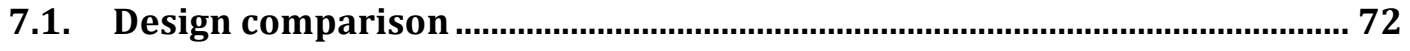

7.1.1. Comparing the arrows design with the central circle design .......................... 72 
7.1.2. Comparing the Arrows and Central Circle Designs with the Cheat Sheet

Design

7.2. Effect of the visual feedback designs during each task ................................... 76

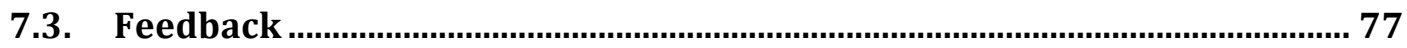

7.3.1. Feedback for correct bend gestures …............................................................... 77

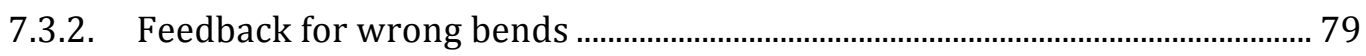

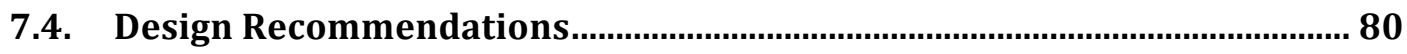

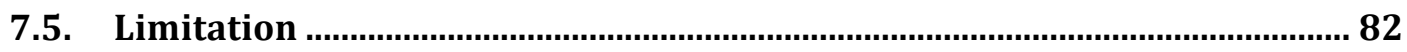

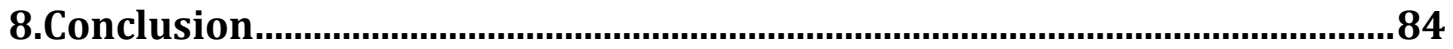

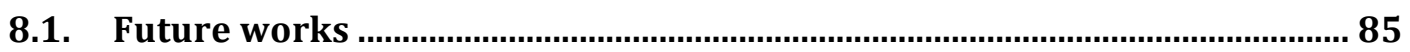

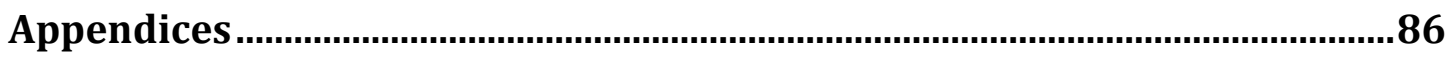

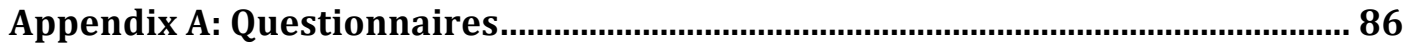

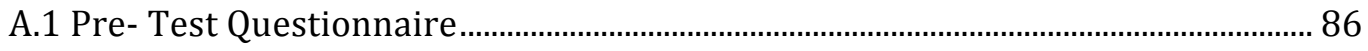

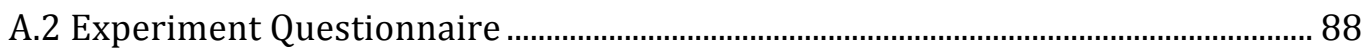

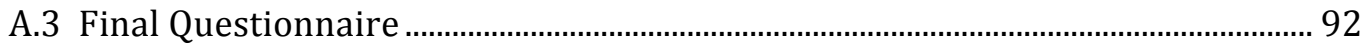

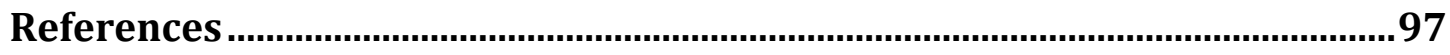




\section{List of Figures}

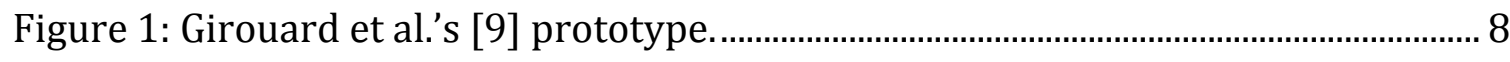

Figure 2: Warren et al.'s [35]classification of bend gestures: (a) location, (b) direction, (c) size, (d) angle, and (e) edge. Size and angle illustrate 3 magnitude levels. 11

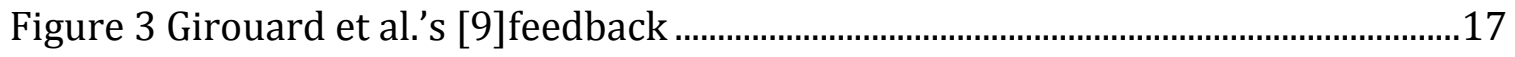

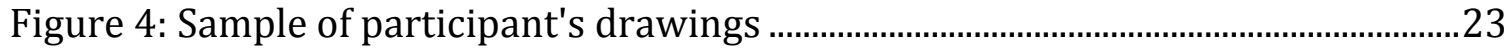

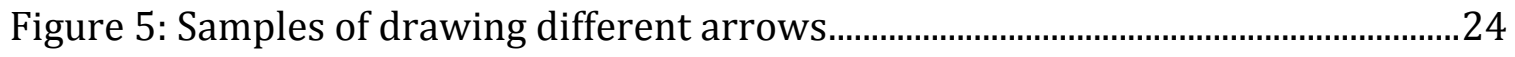

Figure 6: Using blue and red colors to show directions ...................................................25

Figure 7: Using a circle to show activation points of bend gestures ...............................26

Figure 8: Using curve lines to show dual bend gestures ...................................................27

Figure 9: Using circles similar to speedometer to show the angle or pressure ...........28

Figure 10: A sample of the central circle design. ...................................................................33

Figure 11: Using a blue outward pointing arrow located at a 45-degree angle indicates the top-right-down bend gesture. The text "- Size" below the arrow shows the result of performing the top-right-down bend gesture, which in this case decreases the size of an image. .35

Figure 12: Using an orange arrow located at 225 degrees indicates the bottom-leftupward bend gesture. The text "Down" shows the result of performing the bottom-left-upward bend gesture, which in this case is moving down. 36

Figure 13: Using two two-lined orange arrows to show a vertical-upward bend gesture. The words "Right" show the result of performing a vertical-upward bend gesture, which in this case is moving right. 
Figure 14: Half and Full activation

Figure 15: Feedback mechanism of the central circle design. A) half activation, B) full activation, and C) feedback for wrong bend gestures. 40

Figure 16: A sample of the arrows design.

Figure 17: Two sample of showing the feedforward information at the location of the screen that user has to touch or swipe

Figure 18: The top-right-upward bend gesture in the arrows design. The word "Right" shows the result of the bend gesture, which in this case is moving right.

Figure 19: The vertical-downward bend gesture in the arrows design. The words “+ Size" show the results of the vertical-downward bend gesture, which in this case increases the size of an image

Figure 20: Feedback mechanism in the arrows design. A. Half activation while performing vertical-upward bend gesture. B. Full activation while performing vertical-upward bend gesture. C. Showing incorrectly performed bed gesture with a red arrow. In this case, the bottom-left-upward bend gesture is the incorrectly performed bend gesture. 46

Figure 21: Cheat sheet design.

Figure 22: Size of the prototype in comparison with a Samsung Galaxy S3 smartphone

Figure 23: Front and back side of the prototype 50

Figure 24: A Participant at the middle of an experiment...............................................54

Figure 25: Number of trials for each visual feedback design in each task.....................55 
Figure 26: Task 1 (Image docking) .57

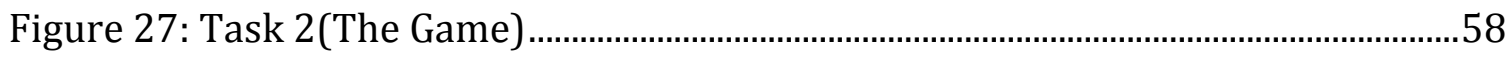

Figure 28: Completion time of image docking task in each design. (N-F= No

Feedback, W-F= With Feedback, W-F-C= with central circle feedback, W-F-A= with arrows feedback). Error bars represent confidence interval 62

Figure 29: Number of errors during performing image docking task with each design (N-F= No Feedback, W-F= With Feedback, W-F-C= with central circle feedback, W-F-A= with arrows feedback). Error bars represent confidence interval.........64

Figure 30: Completion time of the game using each design. (N-F= No Feedback, W-F= With Feedback, W-F-C= with central circle feedback, W-F-A= with arrows feedback). Error bars represent confidence interval 65

Figure 31: Number of errors during performing the game in each design. (N-F= No Feedback, W-F= With Feedback, W-F-C= with central circle feedback, W-F-A= with arrows feedback). Error bars represent confidence interval ..........................66

Figure 32: Level of user's preference for each feedforward design ..................................68

Figure 33: Designs' ranks in terms of visually appealing.................................................69

Figure 34: Designs' ranks in terms of containing useful information .............................70

Figure 35: Information becoming obscured in arrows design. ........................................74

Figure 36: Using rectangle instead of circle...........................................................................74

Figure 37: Central circle design covering the tasks' contents..........................................78

Figure 38: moving the shapes from under the user's thumbs ........................................81 


\section{Introduction}

\subsection{Overview}

Throughout recent years, we have observed an increase of handheld computing applications. It is predicted that by 2016, more than 2 billion people will use these devices, which would further increase to 2.56 billion by 2018 [5]. Due to this large demand and given the rapid advances in technology in handheld devices, there is an increase of interest in research on new methods for the fabrication and interaction with handheld devices. One of the technologies that has captured the interest of many scientists is the technology of handheld flexible devices that allow their users to use bend gestures as a form of interaction.

In general, research on flexible displays can be classified into two groups:

1- Research focused on hardware challenges and problems $[1,2,24]$. In this group, scientists have always been searching for suitable materials for application in flexible displays. Finding proper materials to fabricate flexible batteries, displays, and chassis is a challenge still in need of further research.

2- Studies about interaction with flexible devices $[10,12,13,28,31]$.The aim of this group of researches is to find methods for better and simpler interaction with such devices.

A close investigation on the research in the second group revealed that most studies investigated or proposed user interaction by means of bending $[13,14,19,23,24,28,35]$. In general, designing a proper interaction for communication with a device with small display such as smartphones and tablets 
has always been a major challenge for $\mathrm{HCI}$ researchers. In small devices, since there is a small space for interaction and showing the patterns and shapes, use of the traditional methods such as buttons, menus and the like, has less efficiency [28]. Since flexible devices offers a new way of interaction (bending), many researchers used bend gestures as their main interaction style with flexible displays.

Upon reviewing the literature on flexible displays, we found little information about educating and guiding users in the correct application of a bend gesture. Suppose that a user wants to take a photo with her/his flexible handheld device, which offers bend gestures as its only interaction method. To do this, the user must access the camera application via one or more bend gestures; achieve the proper zoom by means of several bends; and, finally, take the photo. In order to perform all these steps correctly, the user must first know which part of the device she/he should bend. Also, the user must at least learn the correct direction (downward or upward), and the correct angle of the bend. As the bend gestures could differ in their location, direction, size, angle, and speed, for example, learning and performing several bend gestures could be challenging for users to the extent that Lahey et al. [19] found that during their experiment some of the participants found it difficult to even repeat an identical gesture on their prototype.

This research identifies the problems and difficulties a user might experience during her/his interaction with a flexible device, and propose visual feedback systems that offer necessary information in a simple and understandable manner. 
The aim of this research is to help users interact with a flexible device in a simple, flawless manner.

\subsection{Outline of Thesis}

This thesis is divided into eight chapters. The first chapter introduces the topic of visual feedback for flexible displays, our motivations, and the objective of this thesis. The second chapter reviews the previous works related to flexible displays, interactions with flexible displays, and visual feedback systems.

In the third chapter, we discuss our design goals and a participatory design session that we conducted to collect primary information and ideas. The fourth chapter describes the three visual feedback designs that we developed.

The fifth chapter contains the details about our prototype's hardware/software details and information about our experiment, where users were asked to perform sets of bend gestures using our visual feedback designs. The fifth chapter also includes descriptions of our participants and our hypotheses.

The sixth chapter shows our quantitative and qualitative results from the experiment. In the seventh chapter, we discuss our main finding and describe our design recommendations.

In the eighth chapter, we summarize the main findings of this thesis, and we discuss the limitations of our research and provide suggestions for future work. 


\subsection{Contributions}

We contribute to building the foundation of designing visualization guidelines that will help users interact with handheld flexible devices by using bend gestures. In this work, we were interested in exploring different aspects and challenges of providing a visual feedback system that could work on top of different applications and can helps users to correctly perform bend gestures. Using feedforward and feedback mechanisms we developed three visual feedback systems that provide information about location, direction and angle of the bend gestures. We then evaluated these designs in an experiment with two tasks using a flexible prototype.

Our visual feedback designs have proven successful in guiding users to perform bend gestures correctly. Our results revealed information about different properties that a useful visual feedback design should contain. By comparing the results of our designs on users' performances, we found insightful data such as where to locate the graphical designs of the visual feedback on the screen or showing how much detail will be efficient for using in a visual feedback system. In general, this thesis contributes a deeper understanding of how to efficiently guide users interactions with a flexible display using bend gestures. 


\section{Related Work}

Research on flexible displays is a rapidly growing research domain. In order to provide a worthwhile visual feedback design for performing bend gestures on flexible displays, we reviewed related works on flexible displays, interactions with flexible displays, and visual feedback/feedforward for gesture-based interactions.

\subsection{Flexible Displays}

There are different aspects to studying flexible devices, in this subchapter we separate the information in prior works into two general groups: 1 . Information about physical properties and fabrication of the flexible prototypes and devices, and 2. Information about interaction styles and user experiences with flexible devices. We surveyed prior works that have explored these aspects and summarized their findings.

\subsubsection{Physical Aspects of Flexible Displays}

Although flexible displays are not yet commercialized, the technology of flexible displays is advancing rapidly. In the absence of commercialized flexible displays, researchers have come up with novel ideas to create flexible prototypes for use in different studies on such devices.

A group of previous researchers fabricated their flexible prototypes without a screen and instead they used an external display. For example, Balakrishnan et al. [3] introduced ShapeTape, an input device that senses bends and twists. In their research they explored the use of ShapeTape for performing some basic curves, surface creation, and manipulation operations. ShapeTape was a prototype with 
seven optical sensors attached to a spring steel sheet with the size of $960 \mathrm{~mm} \times 10$ $\mathrm{mm}$, connected to an external display. Twend, [12] in an effort to make a hardware prototype that uses bending gestures as input, took a similar approach and fabricated their prototype with external display. They used two $130 \mathrm{~mm}$ x $50 \mathrm{~mm}$ pieces of flexible plastic and embedded optical bend sensors between the pieces to make a basic flexible prototype.

Another group of researchers built more advanced prototypes, which had internal displays. Schwesig et al. [28] built a handheld-sized prototype, called Gummi, using bend sensors and a 2D position controller; both attached to a piece of flexible plastic. In their study, instead of using an external display, they attached a flat-screen display on top of the plastic sheet. Kildal et al. [14] presented the Kinetic device, a fully flexible prototype. They developed their prototype by using a flexible OLED display with two rigid parts affixed to the sides, and used a strain gauge to detect deformation. Wightman et al. [38] in a study for examining Input Techniques for Electronic Book Readers, fabricated a flexible prototype by covering a 6-inch LCD screen with a $203.2 \mathrm{~mm}$ x $177.8 \mathrm{~mm}$ flexible plastic sheet. Tajika et al. [32] examined the influences of physical form factors and the various modes of input for flexible eReaders. Using a larger prototype with a larger piece of plastic and bend sensors, they enclosed a small LCD monitor. Lahey et al. [19] used an active flexible display smartphone-sized prototype using a 3.7 inch electrophoretic display augmented with bend sensors. Their prototype was the first prototype to use an actual flexible electrophoretic E Ink display. 
Another commonly used system for fabricating flexible prototypes in previous studies was using projection to display the materials on to the prototypes. Gallant et al. [8] developed a Foldable User Interface and a prototyping method by using camera sensing and projection. They modified a webcam with infrared LEDs around the lens to detect the markers they put on the prototype. Watanabe et al.'s [36] study is another example of a study that uses projection, infrared reflective markers, and camera. They developed an interface for browsing content that uses the metaphor of turning the pages of a book. Flexpad [31] used a ceiling-mounted depth-sensing camera and projector to provide reliable deformation sensing. In 2010, Ye et al. [39] presented the Cobra, a handheld prototype for computer games with a shoulder-mounted Pico projector. Konieczny et al. [16] developed a handheld flexible display system that allowed intuitive and immersive user interaction with computer generated objects. They used a special spherical lens and a flexible rear projection 9" x 11" screen that users could hold in their hand. Their users could move the screen around the environment and use it as a virtual "slice" to examine the interior of 3D volumes. In a recent study, Girouard et al. [9] developed a prototype with the size of $4 \mathrm{~mm} \times 70 \mathrm{~mm} \times 136 \mathrm{~mm}$. They constructed their prototype out of silicone and augmented it with four Flexpoint 1" bidirectional bend sensors. They also used projection to display the interface on their prototype (Figure 1). Finally, Lo and Girouard [19] presented a set of guidelines to develop a relatively cheap, flexible prototype without the need for expert prototyping skills, that would simulate the functions of a potential upcoming, commercialized flexible devices. They also used projection to display the materials on their prototype. 


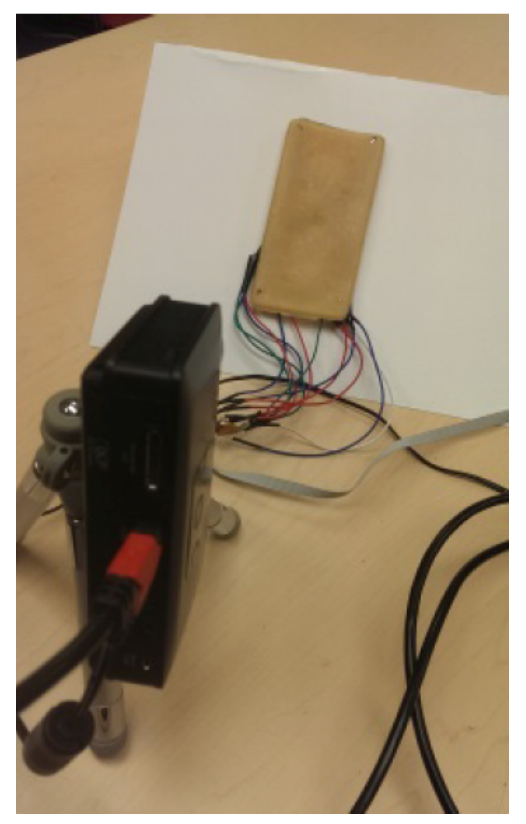

Figure 1: Girouard et al.'s [9] prototype.

In the fabrication process of our prototype, we reused some of the techniques and findings from the above research.

\subsubsection{Interaction with Flexible Displays}

The majority of previous studies on interaction styles for flexible displays focused on bend gestures as the primary interaction style. In this section we focused on development of the bend gestures throughout the past decade and studies that used bend gestures as their interaction style with a flexible display.

Pioneering the flexible display research field, Schwesig et al. [28], in 2003, introduced a set of interaction techniques using bend gestures with their prototype Gummi. Their users could navigate content by bending both sides of their prototype downward or upward. In that research, they integrated single and double bends and used the flat state to distinguish between actions. They concluded that control 
properties of the bending interaction allows types of functionality that would not be possible in a WIMP interface.

In 2008, Twend [12] investigated the use of bend gestures as input for a mobile device. They were interested to measure at least eighteen unique bends, mostly located along the sides and the middle axis of the rectangular prototype. Their bend gestures were: bending the corner of the device back and forth, bending the center of the prototype back and forth along the vertical, the horizontal axis, and finally 2 waveform bend gestures. Two years later, in 2010 Lee et al. [21] explored deformation gestures by categorizing seven types of deformation: bending, twisting, folding, rolling, crumpling, tearing, and stretching. They investigated these deformations on A4-sized plastic, elastic cloth, and paper mock prototypes. Their results showed that users preferred materials with more flexibility for performing bend gestures.

In 2011, with PaperPhone, Lahey et al. [19] evaluated the effectiveness of various bend gestures while performing a set of tasks with a flexible prototype. Their goal was to build a simple interaction language for bend gestures. They classified the bend gestures according to two characteristics: the location and the polarity of the force exerted on the display. Also they defined two sorts of bend gestures: 1. Single bends: the bends that generated from applying force to a singe location of the screen, and 2. Compound bend: the bend gestures that generated from applying forces to multiple locations simultaneously. They found that bend gestures could be a useful interaction style for future flexible devices. Their results also suggested that users preferred conceptually simpler bend gestures along one 
axis that were less physically demanding. Additionally, they suggested that bend gestures form a useful addition to interaction modalities of future flexible computers. In the same year, BendFlip [38] compared five types of input techniques for better interaction in an effort to present design guidelines for flexible e-book readers. In BendFlip study, researchers chose capacitive touch sensors, pressure sensor buttons on the front and back of the display, pressure sensors with onehanded interactions, and three different variations of bend gestures on the side of the display. Their results showed that users could interact with their prototype better using bend gestures rather than using buttons on a flexible e-book.

In 2012, Kildal et al. [14] with conducting a user centered research evaluated bending and twisting interaction styles with a flexible prototype. They proposed a set of design guidelines, including that users perform bend and twist deformations more easily using two hands, especially for larger devices. Lee et al. [20] investigated the effect of size of a deformable device on users' interaction behavior and preferences in 2012. They compared the users' preferences after using two different flexible mock-ups, one $59 \times 115 \mathrm{~mm}$ and the other one $210 \times 297 \mathrm{~mm}$. Their results showed that most users preferred performing bend gestures on the smallsized deformable device.

In a more recent study, Warren et al., [35] in 2013, proposed a classification scheme for bend gestures. They evaluated how users naturally perform bend gestures with minimal instruction. Then, they investigated how users perform bend gestures using four criteria of bend gestures: location, direction, size, and angle (Figure 2). Their study identified two levels of magnitude for bend gestures that had 
optimal distinction with their users with their prototype. They also found that users performed upward bends more than downward bends, and they also recommended using size or angle for clarity with novice users.
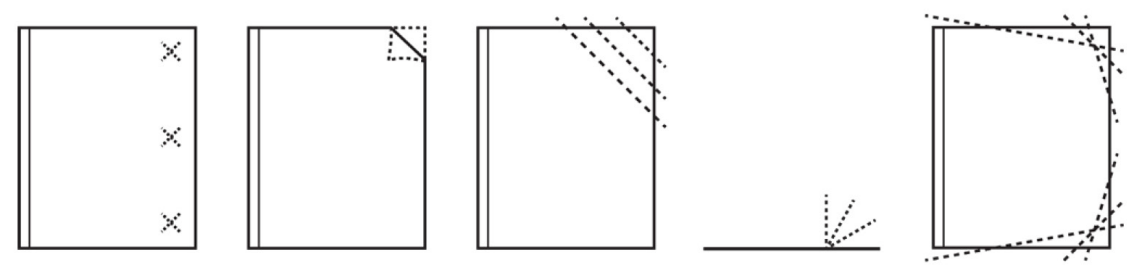

Figure 2: Warren et al.'s [35]classification of bend gestures: (a) location, (b) direction, (c) size, (d) angle, and (e) edge. Size and angle illustrate 3 magnitude levels.

Kildal et al. [15] in 2013, investigated the potential combination of the deformation and touch input in a handheld device. They compared a deformationplus-touch interaction style with a multitouch input technique. Their results showed that users' performed their tasks better using a combination of deformation and a touch screen on the front of the device.

In 2014, Leflar et al. [22] explored the use of bend gestures as the input style to control navigation in 3D virtual environments using a thin flexible device made out of silicone measuring $120 \mathrm{~mm} \times 165 \mathrm{~mm} \times 4 \mathrm{~mm}$. They compared two sets of bend gestures to control a first person camera in a 3D maze. Their results showed that participants preferred the top corner bends to manipulate effectively as part of a navigational control. Finally, Girouard et al. [9] in 2014 explored one-handed deformable interactions for flexible smartphones. They proposed and evaluated a set of deformable gestures for one-handed interactions with a flexible smartphone. Their results showed promise for one-handed gestures and warrant further 
exploration. They also found that the two best gestures were bending the top right corner of the device upward and squeezing the center of their prototype upward.

After reviewing the history of development of bend gestures, we applied the prior work's findings to our study by leveraging mostly from Warren's classification scheme, as it was important to take in to account different factors of a bend gesture.

\subsection{Visual Feedback/ Feedforward}

Research on the field of visual feedback exists since the beginning of the computers and, indeed, the early days of psychology [27]. As Ben Shneiderman mentions in his book Designing the User Interface [29], "For every operator action, there should be some system feedback." To narrow down our findings, in this section we will discuss the studies on visual feedbacks for gesture base interactions. We separate the prior works to two groups: 1.Visaul feedback/ feedforward on rigid displays 2. Visual feedback/feedforward on flexible displays.

\subsubsection{Visual Feedback/Feedforward on rigid displays}

We began our exploration by investigating two important concepts of visual feedback, "feedforward" and "feedback." We also reviewed the research that tried to take a deeper approach to these concepts and to the fundamentals of guiding users to interact with computer devices.

The first research that actually used "feedforward" as a term was Djajadiningrat et al. [6] in 2006. They defined feedforward as a mechanism that informs the user about what the result of his/her action will be, whereas they defined feedback as a mechanism that informs the user about the process of an 
action. In their research they concluded that feedforward and feedback would have far reaching effects for the look and feel of the future electronic products.

Kurtenbach et al. [18] proposed an early example of the use of feedforward in gestural interaction. They designed circular menus to help both novice and expert users perform a menu selection by either popping up a pie menu, or by making a straight mark in the direction of the desired menu item without popping up the menu. Their pie menu was a feedforward display that helped novice users who hesitated when they were unsure of a gesture of command. Their feedforward system proved to be three times faster than ordinary pull down menus [17] and was adaptable for text entry [26] and multiple command entry [11].

Vermeulen et al. [34] investigated the importance of feedforward as a powerful tool to bridging Norman's Gulf of Execution (the difference between the user's intentions and the allowable actions) in designing interactions. They mentioned that, although feedforward was of equal importance to feedback, feedforward was not given as much as consideration as feedback principles. They also believed that although designers do use feedforward in many cases, they do so unknowingly. They reframed feedforward and explained the difference between feedforward and feedback for visual feedback designers.

In addition to reviewing the above papers, to gain more knowledge about implementing the feedforward and feedback concepts we reviewed some research that actually used feedforward and feedback mechanisms to help users learn gesture-based interactions. 
Bau and Mackay [4] described a dynamic guide that combines on-screen feedforward and feedback to help users learn, execute, and remember gestures. Their concept consisted of dynamic guidelines for performing a set of gestures on a touch screen display that uses dynamic feedforward and feedback to directly guide a novice user's performance. By using feedforward mechanisms, they provided information about the gesture's shape with a particular command before users start a gesture. Their feedback mechanisms provided information about the process of recognizing bends during or after the execution of the gesture. They classified their feedforward system in two dimensions: 1 . level of detail-from a minimal hint, to a portion of the gesture, to the whole gesture; and 2. update rate-from only once prior to execution, to discrete intervals, to continuously during execution. Their results showed that users can better learn, execute, or remember gesture sets if they could constantly see the process of the current state of recognition using dynamic guidelines that update constantly according to every action that users make. Additionally, for the level of detail in feedforward, their results showed that users had better performance with a quick hint as feedforward rather than a help menu. Wigdor et al. [37] presented "Ripples", a system to visualize every contact point on a touch screen display. Ripples consisted of a set of six visualizations spanning fourteen states and transitions that place the information beneath and around users' fingertips. Within these visualizations, their users could see the feedback about successes and errors of their touch interactions. Their visualization system was designed to work on top of every application - with no need for any modification in any way-and function independently of the application's responses 
to user input. They mentioned some situations that users might need feedback such as the user might place two fingers onto an object, anticipating a particular response, but another is presented or no response appears at all. They defined a need for visualization of several states, and of the transitions between those states.

Freeman et al. [7] in an effort to help users learn multi-touch and whole-hand gestures on interactive surfaces, presented ShadowGuide, a visualization system for indicating the current hand posture of the users using feedforward and feedback mechanisms. They implemented their gesture leaning tool on a Microsoft Surface, which provided on-demand assistance to the user by combining visualizations of the user's current hand pose as interpreted by the system (feedback), and hand poses and completion paths necessary to complete the gesture (feedforward). Their findings showed that their feedback and feedforward mechanism helped users to learn the most important aspects of a gesture, as well as the effect of changing posture on the recognition process.

Vanacken et al. [33] introduced a visual guideline to help users find available interactions on a multi-touch interface in public spaces. They were interested on designing reconfigurable strategies to decide how or when visual feedback should be activated and which particular visual guide should be presented to the user. They suggested that while it might be impossible to always predict what the user is trying to achieve, a strategy could attempt to assist the user after an erroneous action and it can recommend the most likely guides to the user. 
To gain more insightful information on visual feedback systems, we continued our investigation by reading the previous studies about visual feedback on flexible displays.

\subsubsection{Visual Feedback/ Feedforward on Flexible displays}

To the best of our knowledge, there are no prior studies focusing specifically on providing visual feedback that serves to guide users in performing bend gestures on handheld flexible displays. Previous studies mainly provided verbal feedback to educate their users about the interaction styles. Additionally, most previous studies had a specific visual feedback system, designed to show users the information they needed to complete the tasks of their experiments. Girouard et al. [9] for example, showed the information about the completion of each bend gesture to their users by using two simple visual feedback designs: 1 . a green check mark that appears after successfully completing a bend gesture; and 2. empty circles that would become green after performing a bend gesture (Figure 3). In a study on evaluating use of bend gestures for authentication on mobile devices, Maqsood [25] visualized the activation of performed bend gestures by showing an asterisk in the password field and an LED light on the screen. Moreover, in a different section of their study, pictures of gestures were also displayed to guide users regarding which bend gesture they should perform, and once a user performed a bend gesture, the computer program indicated it by highlighting it. Additionally, Lo [19] used a simple visual feedback system to educate the users about correctly performing bend gestures. For example, when a user was correctly performing a downward bend gesture on the top-right-corner of their prototype, an orange block on the top right 
quadrant would appear, and if the bend were in the other direction (upward) the color would change to blue.

A.

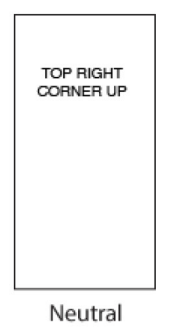

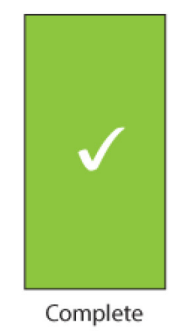

B.

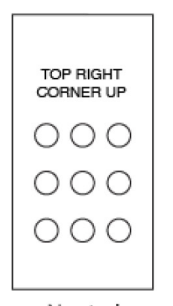

Neutral

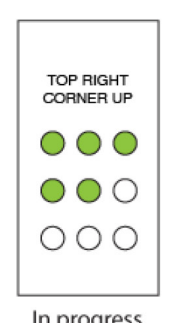

In progress

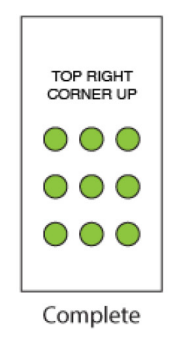

Figure 3 Girouard et al.'s [9]feedback

By combining the information from the fields of visual feedback and flexible displays, in this thesis we aim to build the foundation of providing a visual feedback for performing bend gestures. 


\section{Design Goals}

An important feature of bend gestures is their large number of degrees of freedom [35], and with the development of research in the flexible displays field, researchers have been focusing on wider ranges of bend gestures. For example, in one of the first studies about bend gestures on flexible smartphones, "Gummi: User Interface for Computers," Schwesig et al. [28] used only two bend gestures (bending two corner of the device at a same time up or down). However, in newer studies, such as Bookisheet [36], the number of bend gestures reached up to thirty-six. Similarly, in PaperPhone [19], they identified twenty-four unique bend gesture pairs from a total of eighty-seven collected bend gesture pairs from their participants. This diversity in bend gestures created the need for a method of classification of bend gestures in terms of their features. Warren et al. [35] classified bend gestures by dividing the elements of a bend gesture into seven categories: location, direction, size of the bent area, edge, angle, speed of bend and duration of bend.

For performing a correct bend gesture, depending on the different interfaces, knowing all or part of the mentioned elements is critical for users. However, without a visual feedback system users might run into several problems and difficulties. We began our research by identifying the possible problems that might arise during users' interactions with flexible displays.

We must note that different researchers chose to investigate specific factors of bend gestures according to the aims of their research. For example, Herkenrath et al. [12] used location and direction of the bend gesture as main factors in their research. In this study, we augmented the basic classification of location and 
direction used in majority of the studies $[9,12,14,19,28]$ and included angle to add additional level of complexity to provide more detailed information to the user.

\subsection{Potential problems during performing bend gestures}

Several difficulties could occur during a user's interaction with a flexible display, such as bending a wrong location of the display or bending in the wrong direction. To prevent such problems, the user should be provided with simple and understandable instructions. However, even with clear instructions, a mistake in a bend is still possible. Thus, the user should be provided with additional guidance to help her/him determine their problem and correct it.

We can divide all the information required for interaction with a flexible display in to two major groups.

1- The information a user needs before performing a bend.

2- The information a user needs during or after the bend action.

As mentioned before, there are two mechanisms for showing the users this information and guiding the users to correctly perform the gestures in a gesturebased interaction; Feedforward and feedback [4].

1. Feedforward is the mechanism that presents the required information for starting a command before the user starts to perform it [4]. For example, the word "send" on a button that will do the sending function on an email application shows user the result of clicking that button before the user starts clicking.

2. Feedback is the mechanism that presents the information about the recognition to the user during or after performing the command [4]. For example, 
after hitting the send button and sending the email, "The message has been sent" will pop up on the screen.

In a simpler way of explanation, in a feedforward mechanism the needed information is presented before the gesture is performed, while in feedback they are presented during or after the gesture is performed.

The first challenge in interacting with a flexible smartphone is finding the location of the bend. In other words, to start a bend gesture correctly, a user must first know the location that has to be bent. Besides finding the correct location, the other issue is determining the correct direction of the bend gesture. It means the user has to recognize that she/he must perform the gesture in which direction (up or down) for activating a bend gesture. We can conclude that, before starting a bend action, a user must know at least the appropriate bend location and bend direction.

It must be noted that in this study, by up direction we mean the direction that the user has to bend the device toward her/his face, this direction could be referred as inward since the bend is toward the user. Also by down or outward direction, we mean the direction that the user has to bend the device in the opposite direction of her/his face.

After the user starts a bend, further information about location and direction is not required; the user has already made those decisions. At this stage, the only necessary information is how much pressure must be exerted on the device in order to activate or terminate a bend. Simply put, the user needs to know how far the device must be bent. This parameter is called angle of the bend. 
Knowing these three parameters - location, direction, and angle - the user can perform a bend gesture from beginning to end. If all these steps are done properly, the bend gesture is performed correctly. However, while performing a bend gesture user might apply the wrong angle, the wrong location or the wrong direction. To resolve such problems and educate the user about her/his mistakes, users should have additional information; that will help the user revise her/his decisions.

After clarifying the mentioned basic concepts on flexible displays and visual feedback, to reach to a deeper understanding, and start the design process of visual feedbacks for flexible smartphones we held a participatory session.

\subsection{Participatory Design Session}

After collecting the initial data from the literature, we decided to use a data collecting method in human-computer interaction field called participatory design session. In this method, researchers gather a group of people who can be possible future end users of a device, and with data collecting methods such as brain storming and collaborative designing seek to find insightful information [40].

We assembled five people (three men, two women) with an average age of 24 years. All the group members were university students with no special skills in the field of flexible displays.

At the beginning of the session we explained the research objectives with the following simple examples. First, by using a touch screen smartphone, we explained 
the basic concepts such as feedback and feedforward. For an example of the feedforward mechanism, we showed that in an Android cellphone, for unlocking the smartphone's display, a lock sign on the displays indicates the place where the user should touch. Then we explained that the unlocked lock after performing the unlocking command is an example of feedback mechanism. Then we explained the basic and key concepts of flexible devices and bend gestures.

Since the aim of this session was to find novel ideas, we did not provide excessive explanation; we did not want to direct the subjects' thoughts. After our introduction, design tools (pencils, paper, markers, etc.) were presented to the participants, and they were asked to draw whatever came to mind. The following are some of their illustrations (Figure 4). 

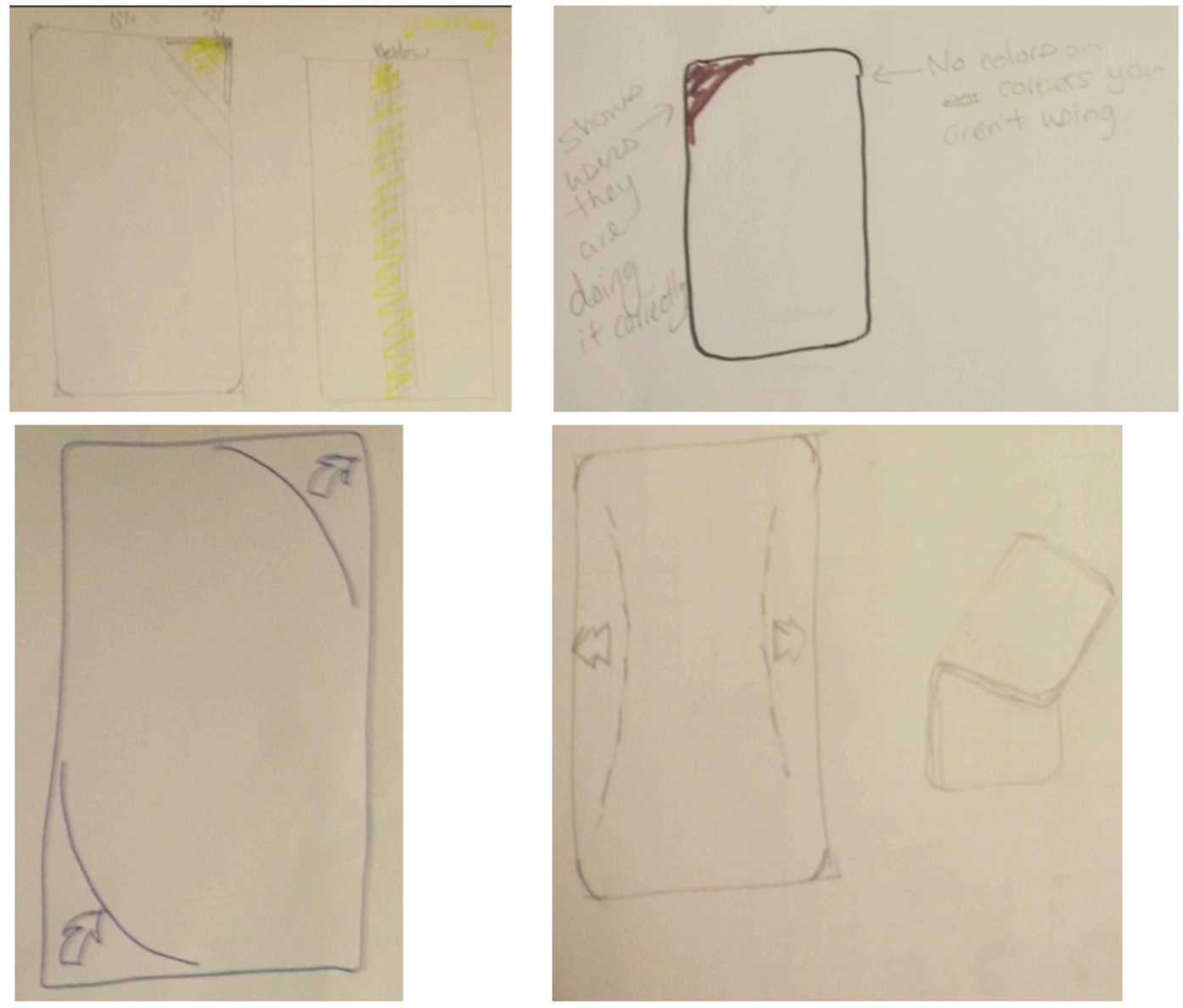

Figure 4: Sample of participant's drawings

\subsubsection{Analysis}

We observed and analyzed participant's discussions, drawings and notes in order to gain insight on different aspects of designing visual feedback for flexible displays. In this section, we discuss the results and extract our main findings.

\subsubsection{Use of Arrows}

One of our main findings was the use of arrows to show various information. Although all the arrows did not have the same shape or location, 4 of the 5 participants somehow used arrow shapes in their designs (Figure 5). For example, 
one of them used a circular arrow for illustrating angle, and another participant used arrows to indicate the direction of the bend gestures. Most of the arrows were used to indicate the place or direction of the bends. One possible reason for using arrows at this level might be that the arrow sign is a simple international symbol for direction, employed in many fields and in daily life (road signs, for example). Hence, we hypothesized that the use of arrows in visual feedback could be helpful.
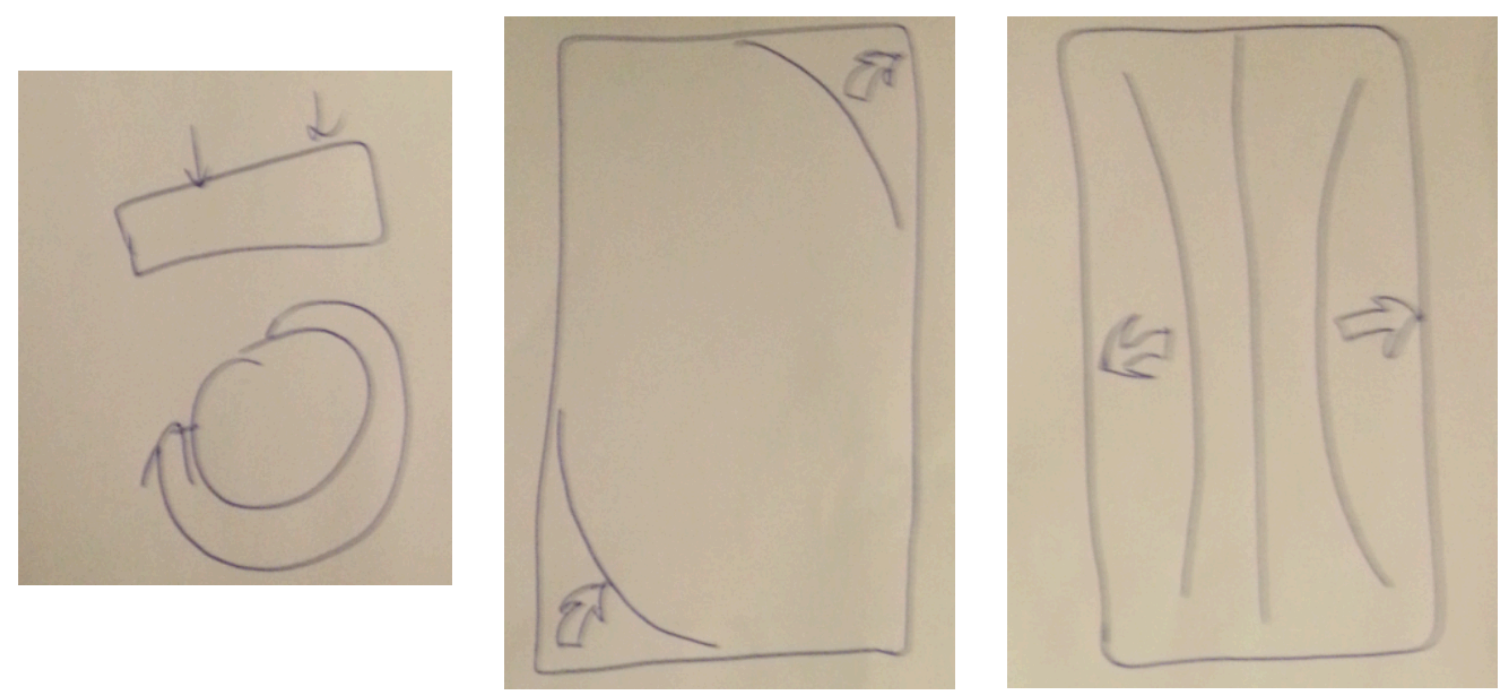

Figure 5: Samples of drawing different arrows

\subsubsection{Different Colors}

Our second observation was that three participants used at least two colors in their designs and the percentage of use of red, blue and green colors were higher than the other colors. For example, one participant applied blue and red colors for showing the up and down directions, and two participants used red color for indicating the errors. Figure 6 shows the use of red and blue color to indicate 
different directions. In this picture, a participant shows the two directions with a thin line of red or blue color.

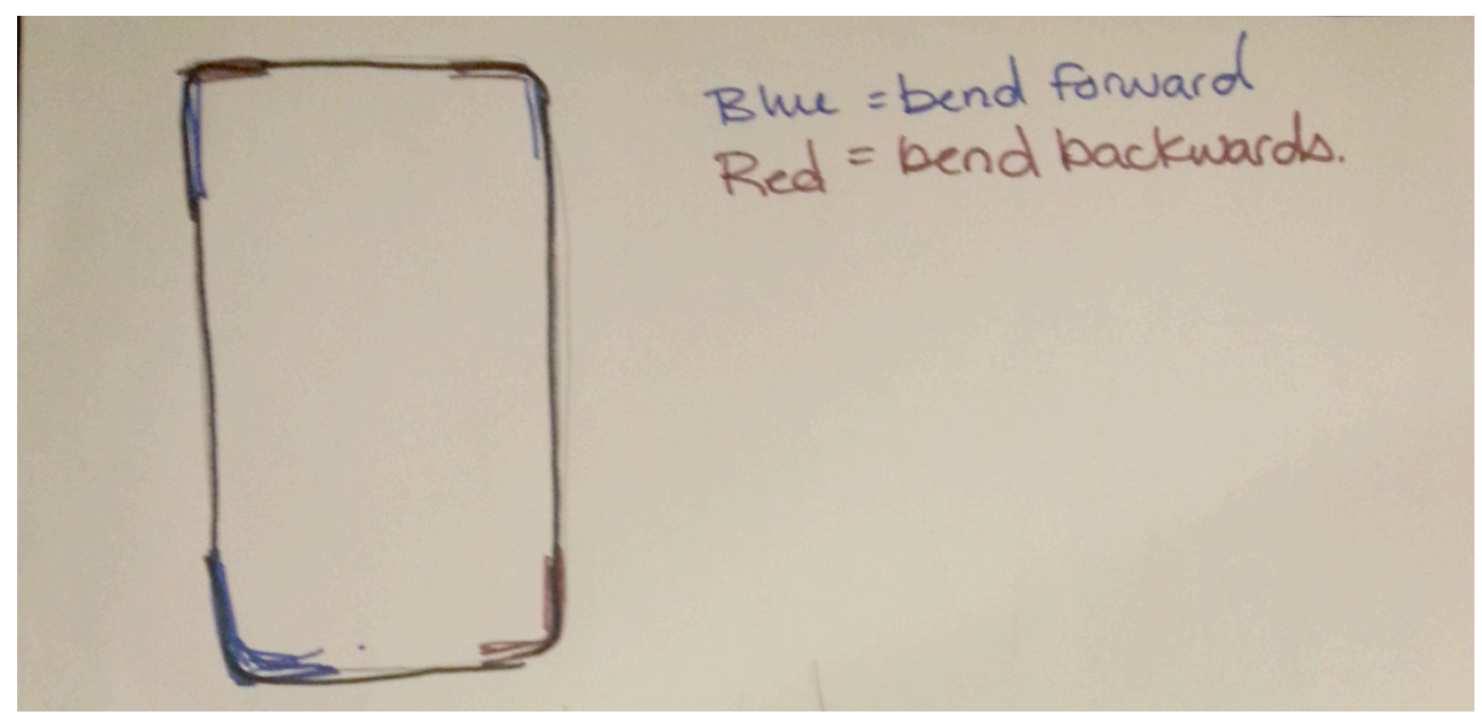

Figure 6: Using blue and red colors to show directions

\subsubsection{Using simple shapes}

We found that the participants applied simple shapes such as circle, semicircle or triangle for showing the different information such as location, direction and angle. For example as it can be seen in Figure 7, one of the participants used circle or semi-circle for showing the level of pressure in performing the bend gesture. An interesting point in the shapes drawn by the users is that they all have small sizes in a way that they do not occupy much space of display. 


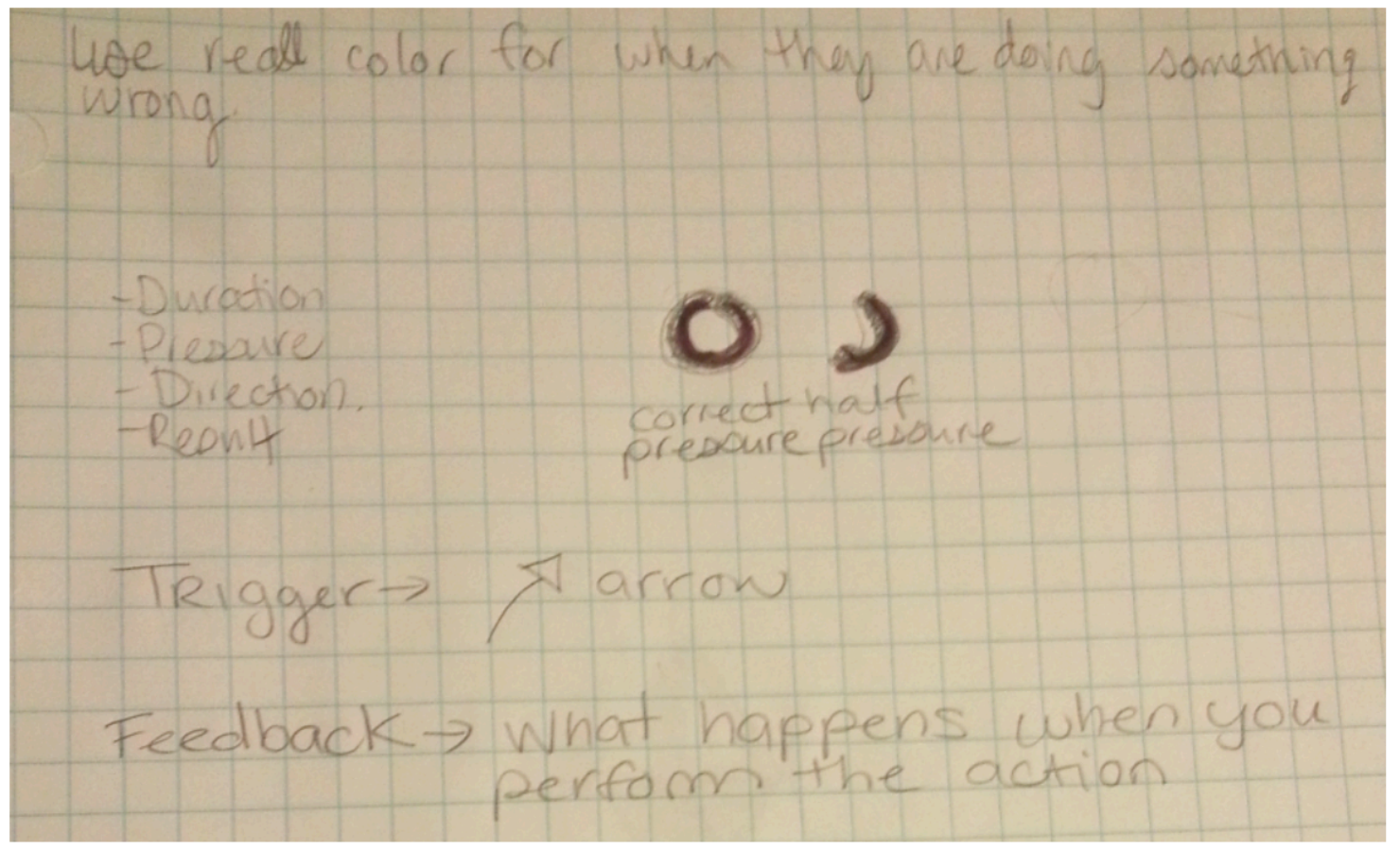

Figure 7: Using a circle to show activation points of bend gestures

\subsubsection{Location of the shapes}

A point addressed a lot during the session was the location of the shapes in the display. Some participants believed that it is better to place the shapes in the bend location or, in other words, the location where the bend has to be performed. However, some other participants thought that the shapes could be placed in locations other than the bend location. For example, one of the participants, by drawing a small circle at the bottom or in the middle of display, believed that the shapes should be placed in locations where they do not interfere with the user during bend performance, while another participant plotted his patterns in the same location as the bend. This duality among the participants shows that further testing of both placement options would be a valuable endeavor. 


\subsubsection{Dual bend gestures}

An important discussion and challenge during the session was how to indicate dual bend gestures, which require two simultaneous bends at two different locations. Some suggested guiding the user with text. For example one participant suggested writing "dual gesture" close to the shapes that indicate the bend gesture. Another participant used two simple lines on the back of the arrows to imply that the bend gestures were simultaneous (Figure 8).
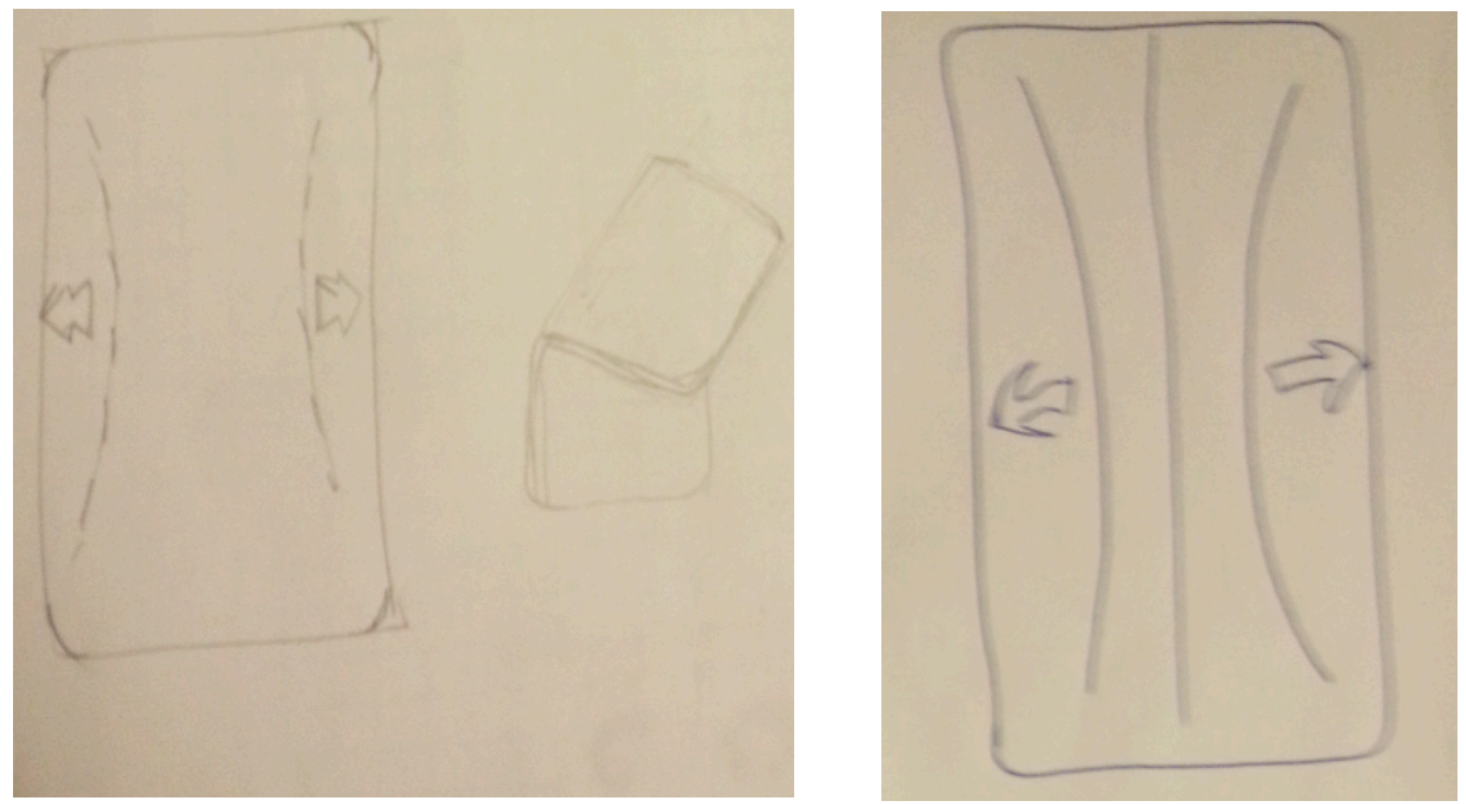

Figure 8: Using curve lines to show dual bend gestures

\subsubsection{Indicating angle}

The final point involved showing the information about angle of each bend. Most participants drew patterns that can also be observed in the real world. For example, one participant's angle indicator was a marker within a circle, analogous to a car's speedometer (Figure 9). On a speedometer, as the amount of pressure on the 
gas pedal increases, the angle of the marker from its origin increases. Another participant designed a horizontal bar, which started empty and filled with green from left to right as pressure increased.
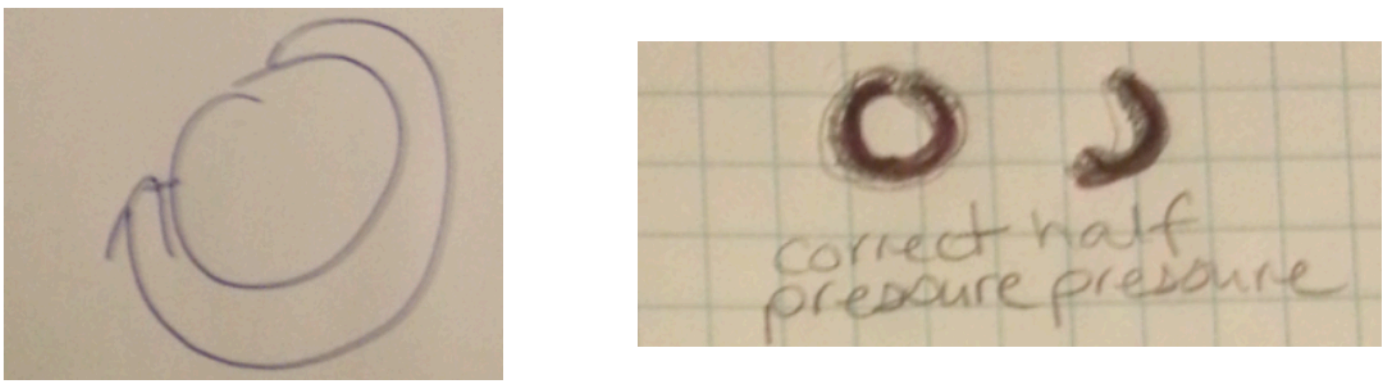

Figure 9: Using circles similar to speedometer to show the angle or pressure

In summary this participatory design session provided us with basic information and ideas to start the process of designing our visual feedback systems. One of the main ideas that can be extracted from this session is using arrows to indicate the direction of bend gestures. Also participants' designs using two different colors for showing the direction of the bends were noticeable. Furthermore, using two curved lines for indicating dual bend gestures grabbed our attention because the concept is easy to understand and it could be an alternative for using text.

After analyzing our results, we came to the conclusion that it would be better to gather a group of people with skills in flexible displays or human-computer interaction. The absence of expertise of the participants in the flexible displays area let to ideas that were simple and lacked depth. 
In retrospect, we noticed that we did not follow the standard participatory design methodology. A better methodology for gathering data would have yielded richer information. According to Spinuzzi [30], a participatory design should consist of three stages:

1. Initial exploration of work: In this stage, designers meet the potential end users and familiarize themselves with the technologies that users are already using in their daily life. Designers gather data about users' opinions regarding problems with the current technologies and their expectations for a better future technology. We could gather these data using interviews, questionnaires and observing users' behaviors while working with their smartphones or tablets.

2. Discovery processes: In this stage, designers and users employ various techniques to understand the idea of the research better. After fully understanding the purpose and goal of their work, they interactively start the design process and cooperatively brainstorm to make meaning out of the work. This stage is often conducted in a conference room with a group of users.

3. Prototyping: Finally, using simple mock-ups or even working prototypes, designers and users investigate their designs interactively. We could conduct this stage using a working prototype to evaluate the designed visual feedbacks in stage 2. Subsequently, we could implement any potential improvements to our designs before finalizing our designs to conduct our main experiment. 
After reviewing our methodology of conducting a participatory design, we found that we had only conducted the second stage of a complete participatory design methodology. Before starting the process of designing and conducting our participatory design session (an example of discovery process), we should have started with gathering initial data and information about users' experiences and opinions on visual feedback designs that they use in their daily lives. Furthermore, before finalizing our visual feedback designs, we should have conducted the third stage (prototyping) to gather more data for potential improvements. 


\section{Designing Visual Feedback}

After analyzing the data from participatory design session, we started the design process of guidelines to help users perform correct bend gestures on flexible displays. We developed three visual feedback designs: 1 . Central circle design, 2. Arrows design and 3. Cheat sheet design. We proposed a novel feedforward and a feedback mechanism for central circle design and arrows design. As we needed a baseline to compare the usability of the central circle design and the arrows design, we developed the cheat sheet design with a minimal, basic feedforward mechanism and no feedback mechanism.

In this chapter, we present the group of bend gestures that we decided to concentrate on, and we present our three visual feedback designs in detail.

\subsection{Gestures}

We selected 12 unique bend gestures to use in this study. We decided on 12 bend gestures because we wanted to have enough diversity while avoiding unnecessary complications such as distinguishing between collocated gestures that could be caused by a large number of bend gestures.

We decided to use the four corners of our flexible prototype, and each corner had upward and downward flexibility. One of our main objectives in this study was to consider the gestures for which activation required the simultaneous bending of two locations. Therefore, we used four spanning bends, which were the vertical upward, vertical downward, horizontal upward, and horizontal downward bends. 
Hence, there were eight corner gestures and four middle gestures for a total of twelve unique bend gestures.

\subsection{Central Circle Design}

The main idea behind the central circle design is to present all the required information that is necessary for a complete interaction with a flexible device, always at one location on the screen. In this concept, the user always knows where to look to find the information she or he needs.

The central circle design contains a circle with a $58 \mathrm{~mm}$ diameter $(30 \%$ of our prototype's screen) and it has two types of arrows (simple and double). In addition to these shapes, it also contains text with two colors to show the result of performing each bend gesture. This design is always located at the middle of the display, and all the information from the feedback and the feedforward mechanisms always appear in this circle (Figure 10). 


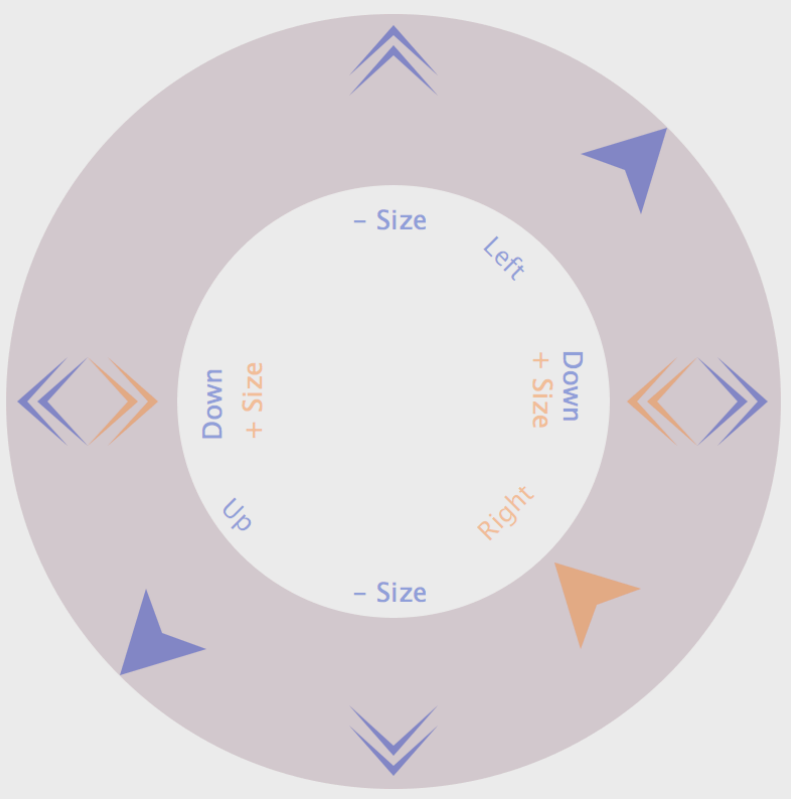

Figure 10: A sample of the central circle design.

We chose to place the circle in the middle of the display for the following reasons: 1 . The distance between the circle and each bend gesture is close, and users can easily see the information in the circle and the location of the bend gesture at the same time. 2. While performing any bend gesture on a flexible device, a part of the screen becomes invisible. In our prototype, the only place that is always visible is the center of the screen.

The main advantage of this design is that, because information always appears in one place on the screen, users always know where to look to find the information. 


\subsubsection{Feedforward Mechanism in the Central Circle Design}

As mentioned, the data in the feedforward mechanism includes what the user needs before starting an interaction with a device. Before the user begins any bend, he or she has to determine its location and the correct direction, and, in addition to location and direction, the user must become aware of the results of the bend gesture.

In this design, we used arrow tips to show the location and direction of each bend gesture. These arrows are located at the edge of the circle, and each arrow represents an available bend gesture. The location of an arrow represents a bend gesture's location, and the direction and color of the arrow indicates the gesture's direction. To show the direction of each gesture, we used arrows pointing in two possible directions, inward (up) and outward (down) as related to the circle's center. The outward arrows were blue, and the inward arrows were orange. For instance, to show a top-right-downward bend, we used an outward-pointing arrow on a circle located at a 45-degree angle (Figure 11). To show a bottom-left-upward bend, we used a blue, inward-pointing arrow located at a 225-degree angle on a circle (Figure 12). 


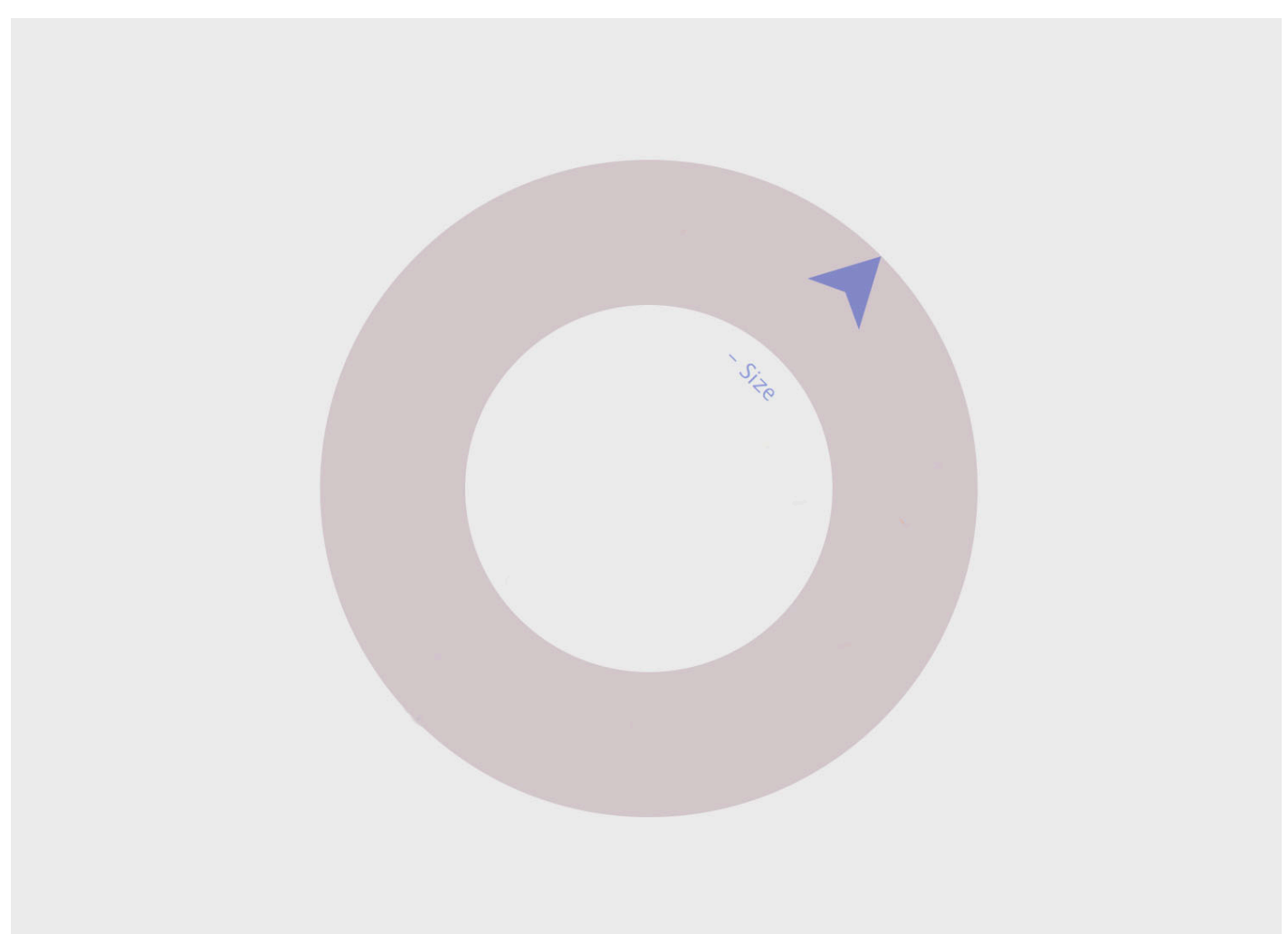

Figure 11: Using a blue outward pointing arrow located at a 45-degree angle indicates the top-rightdown bend gesture. The text "- Size" below the arrow shows the result of performing the top-right-down bend gesture, which in this case decreases the size of an image. 


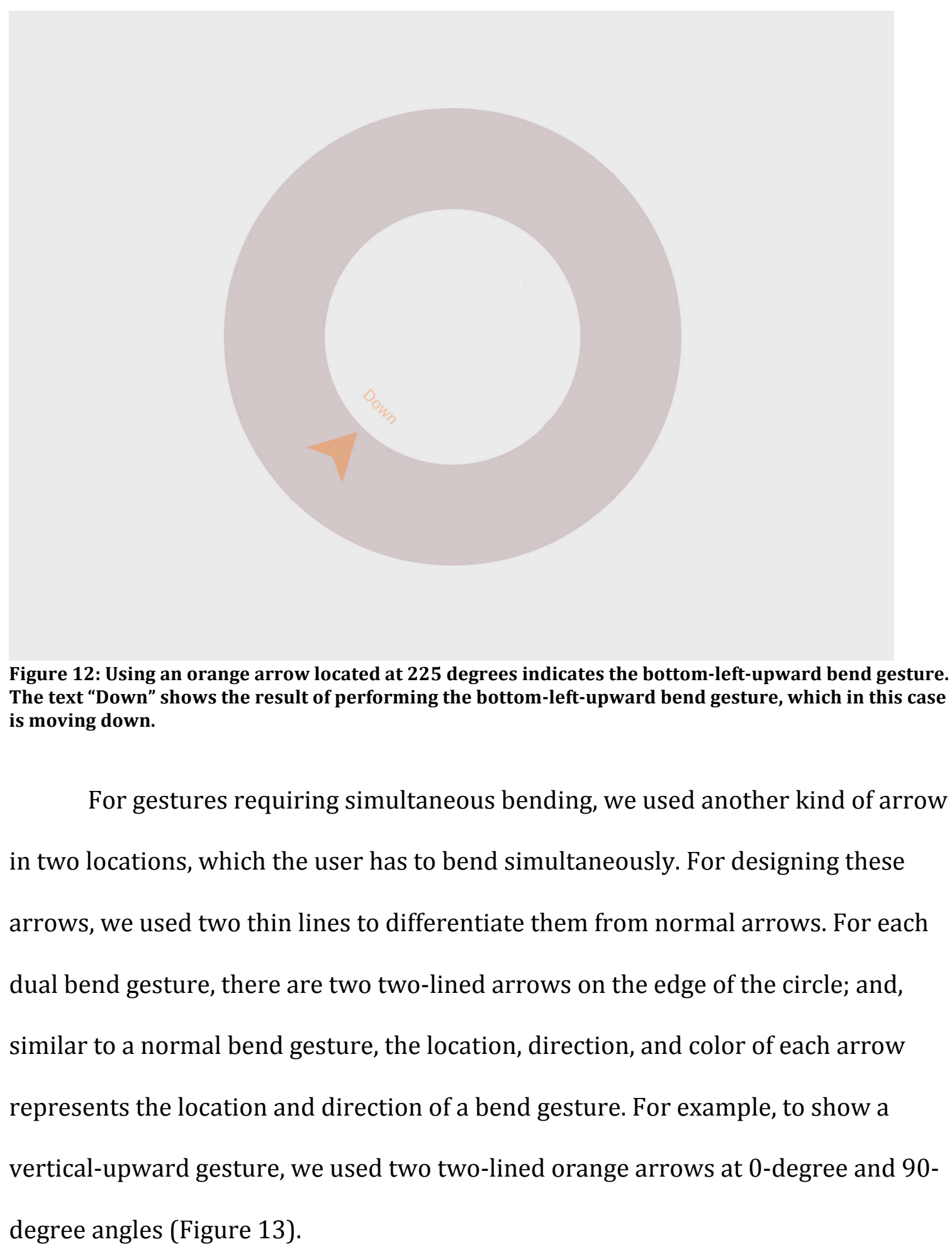




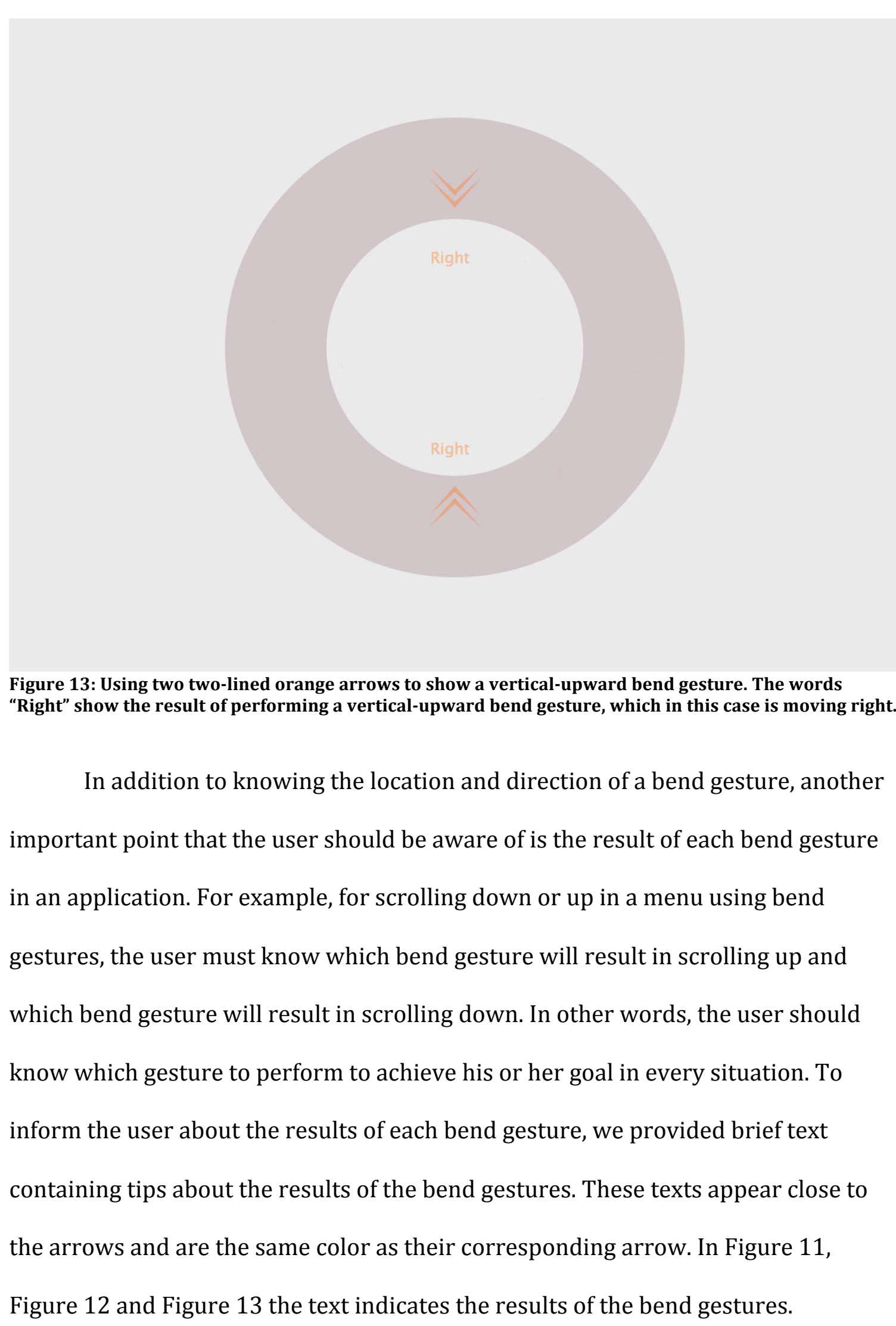




\subsubsection{Feedback Mechanism in the Central Circle Design}

Once users become aware of all three pieces of information in the feedforward mechanism (location, direction, and resulting action), they start to perform a bend gesture. At this stage, the feedback mechanism is needed to help users finish a correct bend gesture or to stop them from performing an incorrect bend gesture. This information helps the user complete the gestures in the best and most accurate manner possible. While performing a correct bend gesture, the feedback mechanism provides information about the angle of a bend gesture. In other words, it shows the user how much they should bend the device to finish a bend gesture completely. For example, if for activating a bend gesture a corner of the device must be bent 40 degrees from its initial position, the feedback mechanism will show the user when she or he reaches 40 degrees.

We applied two angle levels in our designs: half activation angle and full activation angle (Figure 14). Half activation indicates the midpoint of the angle for activating the bend gesture, and full activation indicates the angle that a user must bend the device to finish a bend gesture completely. For example, if the threshold for activating a bend gesture is set to be 40 degrees from the initial position, then the half activation level will be 20 degrees, and the full activation level will be 40 degrees, which is the same as the actual activation threshold. 


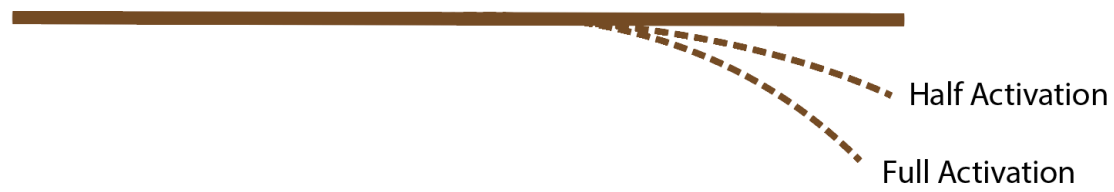

Figure 14: Half and Full activation

In addition to having a feedback mechanism for correct bend gestures, we developed a mechanism for incorrectly performed bend gestures. When a user attempts to perform a bend gesture, it is possible that he or she has made the wrong choice and is attempting to perform an incorrect bend or an unavailable bend. In this design, the display of a red $\mathrm{X}$ at the center of the circle would convey to the user that he or she is performing an incorrect bend (Figure 15). The idea behind this simple feedback was that a cross would not convey any unnecessary information to the user.

We used the empty space in the middle of the circle to show the angle of the bend parameter in the central circle design. Before the bending starts, the internal space of the circle is empty. When the user starts to bend the device, the internal space of the circle remains empty until the exerted pressure reaches the halfactivation level. At that point, half of the central circle turns green. Subsequently, when the user increases the pressure, he or she ultimately reaches the full activation stage. At this stage, the entire inner space of the circle turns green (Figure 15). 

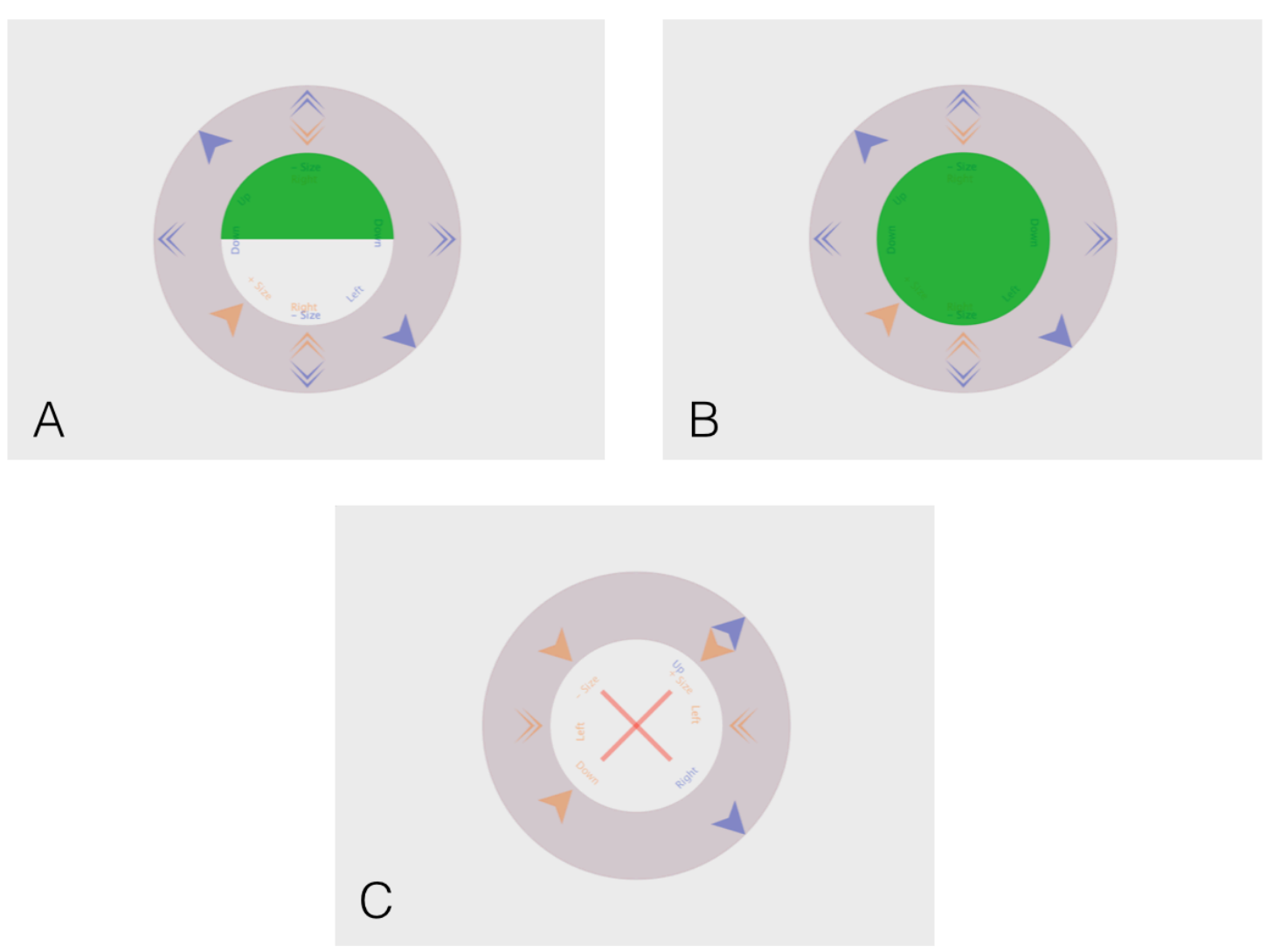

Figure 15: Feedback mechanism of the central circle design. A) half activation, B) full activation, and C) feedback for wrong bend gestures.

\subsection{Arrows Design}

While the central circle was designed so that information is always shown in one area of the display, the arrows design, our second design, presents all the required information for having a complete interaction with a flexible device at the actual location of the bend gesture. In this design, we provide information about each bend gesture in the location that users have to perform the bend gesture. As a result of this concept, in the arrows design, the locations of the shapes and texts are dynamic, and they are able to change according to the available bend gestures in different applications. Figure 16 shows an example of the arrows design. 


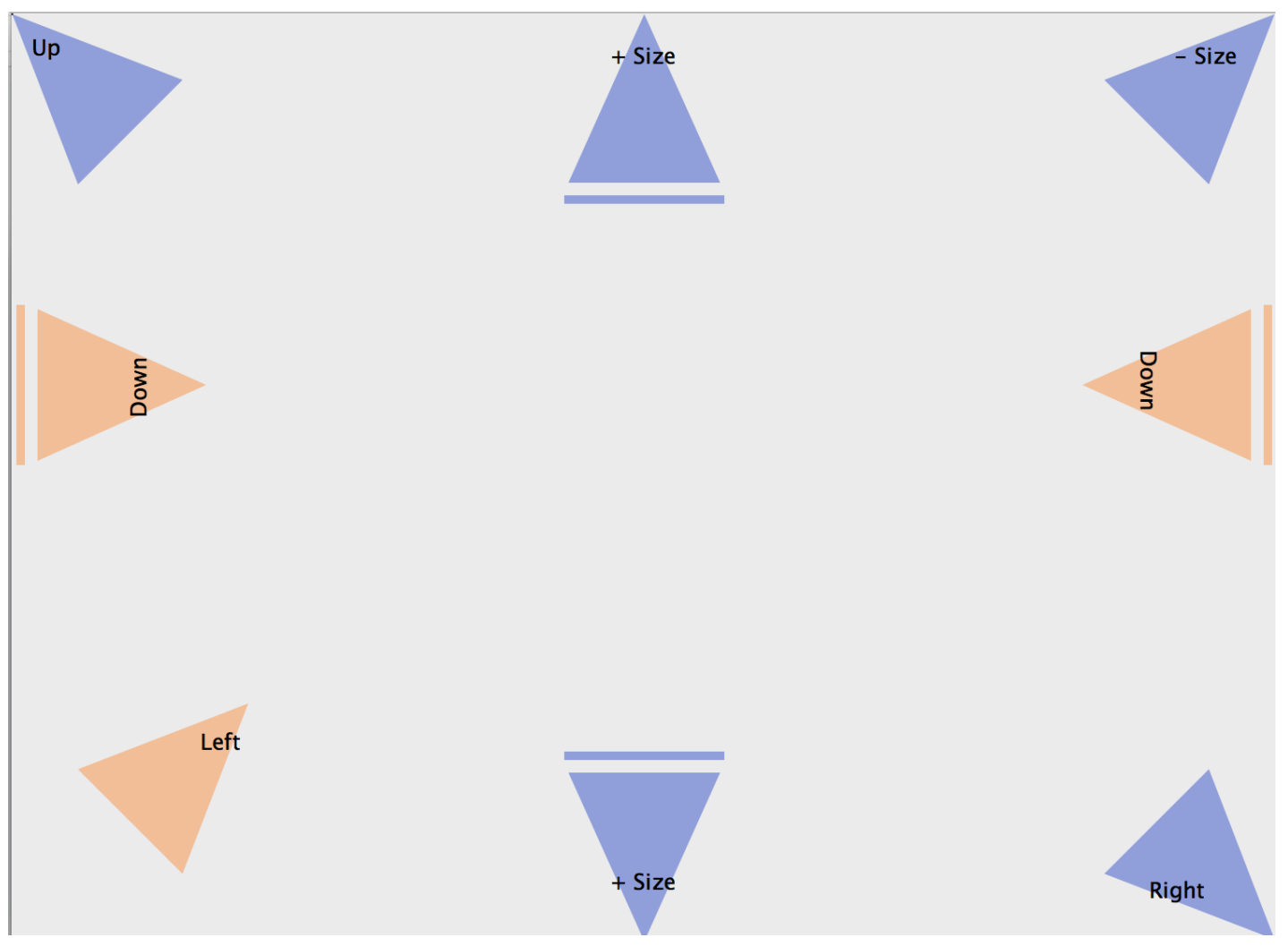

Figure 16: A sample of the arrows design.

We decided to show the information at the actual location of each bend gesture for the following reasons: 1 . We hypothesized that using a direct manipulation technique, which shows the information exactly where the user has to take the action, will improve the user's performance. 2 . The user is able to see the information and perform the bend gesture all in one location, which will result in a faster interaction. 3. The concept of showing information at the location of the actual action is popular in most commercial devices such as touch display smartphones and tablets (Figure 17). 

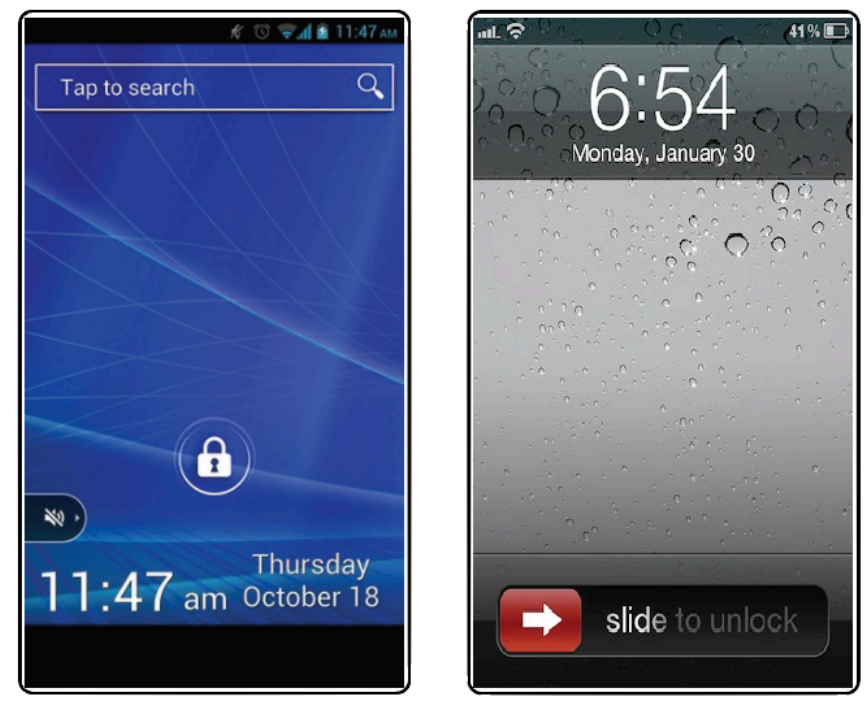

Figure 17: Two sample of showing the feedforward information at the location of the screen that user has to touch or swipe

Similar to the central circle design, we used arrows to show the location and direction of the bend gestures. In this design, in which an arrow represents the exact location of a bend gesture, the user has to bend the exact location of the arrow. Because the only shape that we used in this design was arrow (unlike the central circle design, which contained a large circle), it gives us enough space to make the arrows bigger than they would be in the central circle design.

In the arrows design, the concept of showing the direction of the available bend gestures is similar to the central circle design. An arrow's direction and color represent the direction of the bend gesture. The arrows point in two directions, inward (up) and outward (down); the outward arrows are blue, and the inward arrows are orange. Figure 18 shows an example of the top-right-upward bend gesture in the arrows design. 


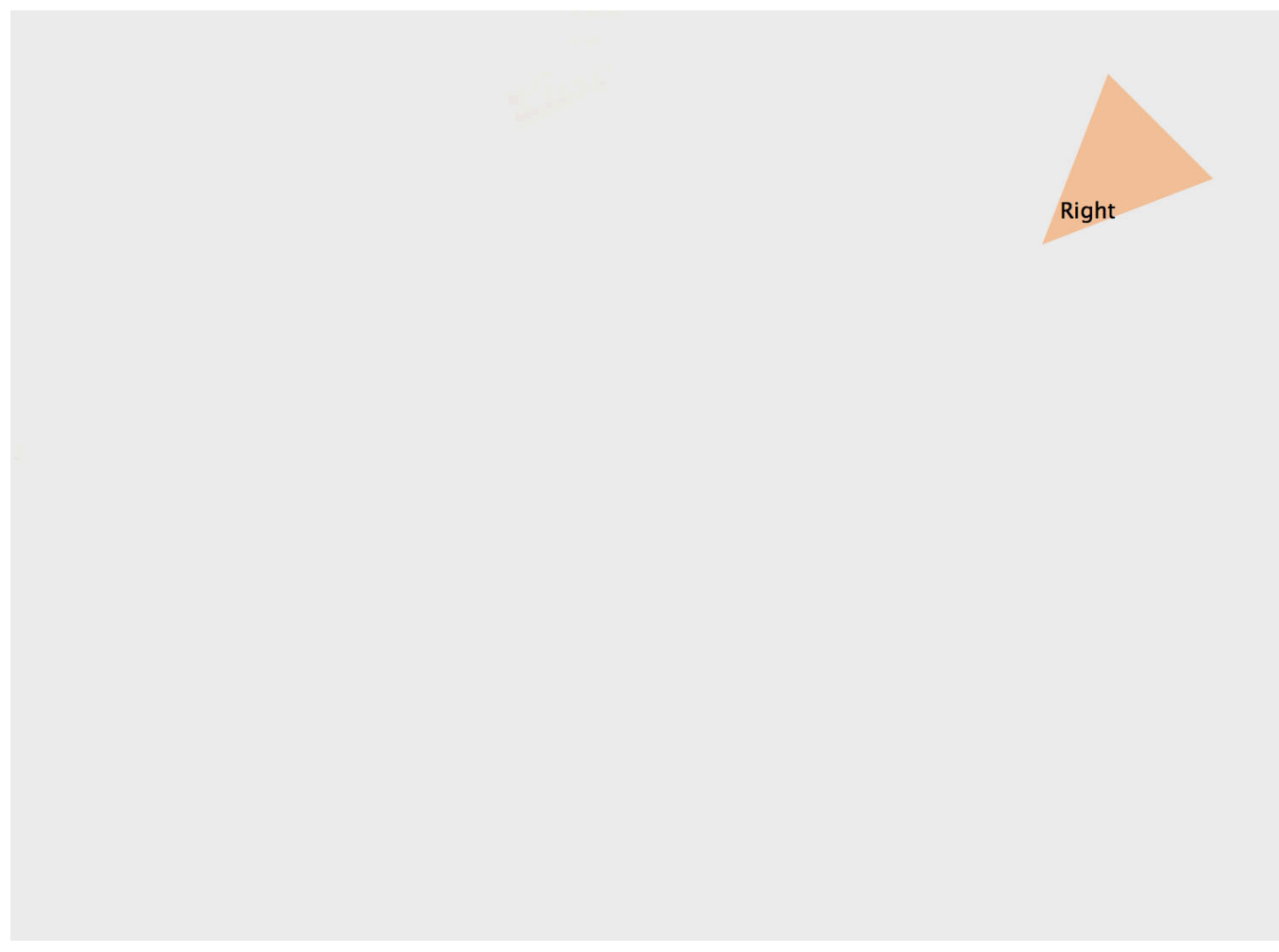

Figure 18: The top-right-upward bend gesture in the arrows design. The word "Right" shows the result of the bend gesture, which in this case is moving right.

For showing a gesture that requires simultaneous bending, we used an arrow with a line at the base of the arrow. For each dual bend gesture, there are two baselined arrows at the locations that the user has to bend simultaneously. We adopted this idea from one of our participants' designs in the participatory design session. For example, to show a vertical-downward gesture, we used two, base-lined blue arrows at the top and bottom of the screen (Figure 19). 


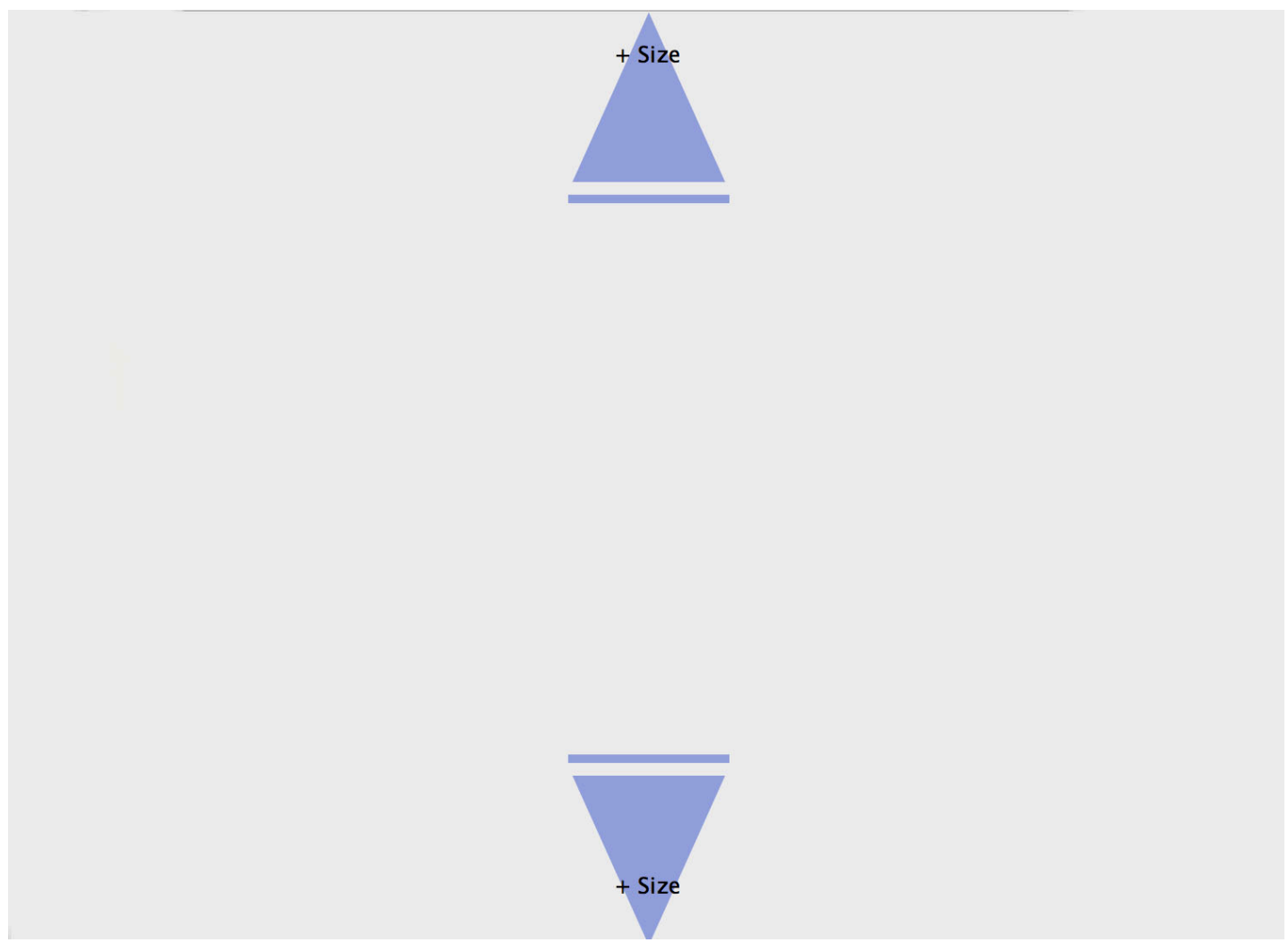

Figure 19: The vertical-downward bend gesture in the arrows design. The words "+ Size" show the results of the vertical-downward bend gesture, which in this case increases the size of an image.

Moreover, to show the results of each instance of bending, similar to the central circle design, we used text. We located the text inside the arrows, closer to the thinner part of the arrows, and the color of the text was always black.

Overall, in the feedforward mechanism of the arrows design, all the necessary information to start a specific, correct bend gesture (location, direction, and result of the action) appears in a small arrow (in relation to the size of the display), which occupies a much smaller area of the screen, when compared to the central circle design. 


\subsubsection{Feedback Mechanism in Arrows Design}

In this design, we used arrows displaying the location and direction of the bends to illustrate feedback. If users start to perform a bend correctly, they are guided to complete the bend using the same two levels of angle used in the central circle design, which are half activation and full activation. At half activation, the widest half of the arrow turns green, as illustrated in Figure 20a, to indicate to users that they need to increase the curve to activate the gesture completely. When users bend the device to such an extent, the arrow turns completely green Figure 20b.

We decided to display the feedback information on the same arrows that we used for the feedforward mechanism because we wanted to show all the information at the same place as the bend gesture. As a result, to perform a correct bend gesture from start to finish, users only need to see the actual bending area.

In addition, in a scenario in which a user begins the wrong gesture, the arrow turns completely red, indicating an error. Also, a user trying to create a bend that is unavailable will see a red arrow in the same location (Figure 20c). 

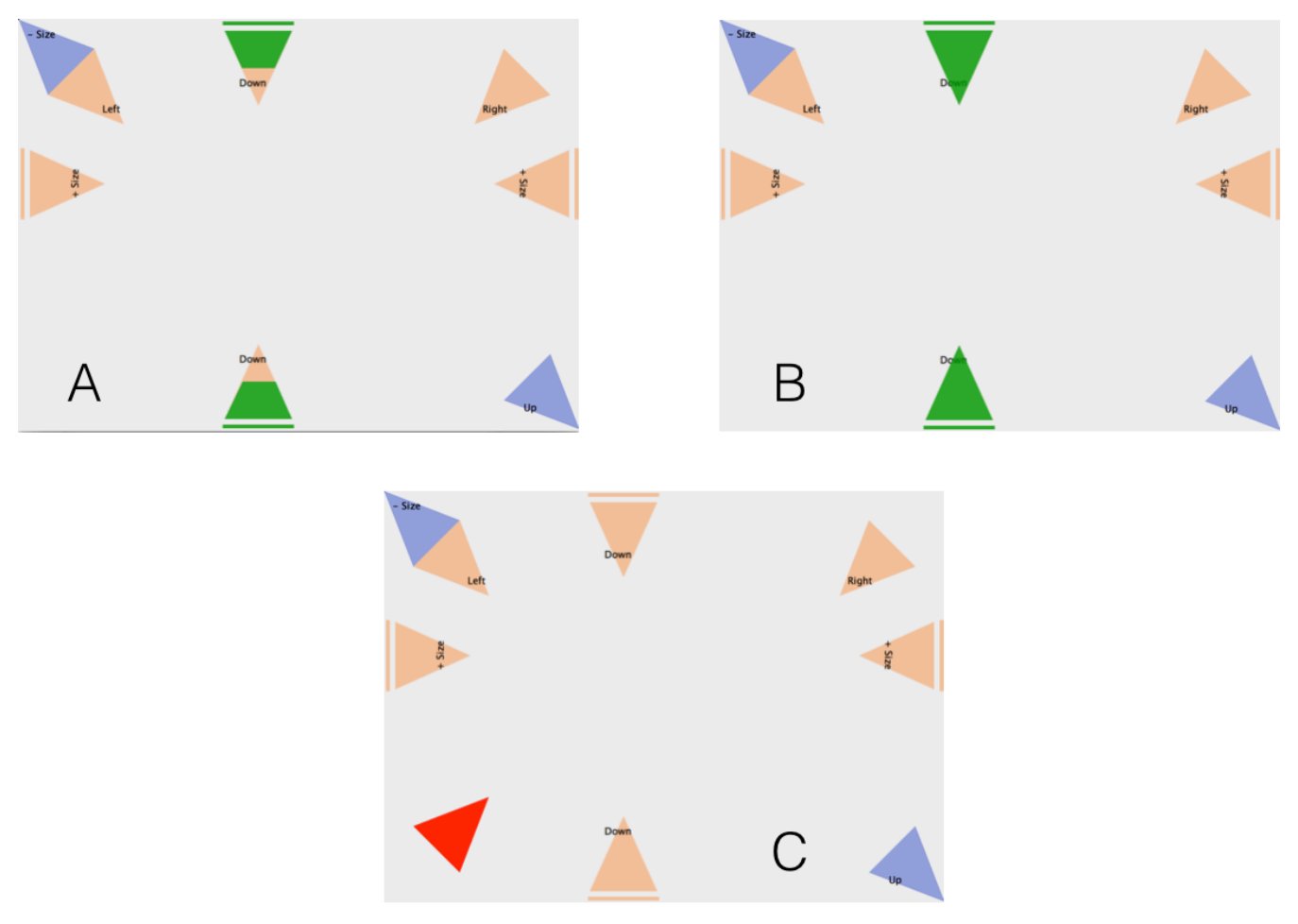

Figure 20: Feedback mechanism in the arrows design. A. Half activation while performing verticalupward bend gesture. B. Full activation while performing vertical-upward bend gesture. C. Showing incorrectly performed bed gesture with a red arrow. In this case, the bottom-left-upward bend gesture is the incorrectly performed bend gesture.

\subsection{Cheat Sheet}

To compare our two designs, we needed an additional, simplistic design that would provide the user with information about the action-to-bend-gesture mapping; yet omit all localized feedforward or feedback information. We chose to present the required information via text.

The main objective of this design was not to present a new, innovative pattern; rather, it aimed to be a baseline by which the two previously discussed designs could be evaluated. As a result, the cheat sheet design is our most basic design, without any geometrical shapes or colors. In this design, we present the 
information in a two-column table at the right corner of the display. The first right column contains the location and direction of each gesture, and the second column shows the result of each bend (Figure 21). We called this design a cheat sheet because it presents all the necessary information for performing a correct bend gesture in a compact style, much the same way as a cheat sheet in real life contains information.

\begin{tabular}{|l|l|}
\hline Move Up & Bottom Right Upward \\
\hline Move Down & Bottom Left Upward \\
\hline Move Right & Top Left Upward \\
\hline + Size & Horizontal Upward \\
\hline Move Left & Bottom Right Downward \\
\hline - Size & Top Left Downward \\
\hline
\end{tabular}

Figure 21: Cheat sheet design.

It must be noted that the order of the gestures in the table is random. As in the two previous designs, all the information is displayed randomly, to ensure and enable an adequate comparison between the designs. 
As we wanted to keep the cheat sheet design very basic, we did not develop a separate feedback for this design. We provided the minimum information needed to start performing a correct bend (location, direction, and the result of the bend gesture), in the cheat sheet table. However, in our experiment we borrowed the feedback of the two other designs to use in cheat sheet design with the goal of comparing the feedbacks in absent of their original feedforward.

Beside our intention to design a cheat sheet that serves as a basic baseline, it must be noted that this cheat sheet design might not be the optimal baseline. A better cheat sheet could represent the information in a non-random order, so users could find the information more easily in the table. In addition, showing the cheat sheet table at the center of the screen instead of bottom right corner might improve users' performance using this design. As mentioned previously, the center of the screen is the only location of the screen that is always visible during performing each bend gesture. 


\section{Experiment}

\subsection{Prototype}

To conduct the user study and testing the efficiency of the designs, we designed and fabricated a flexible prototype that would emulate a real product.

\subsubsection{Hardware}

We considered the size of our prototype larger than the commonly used smartphone and closer to the size of a tablet or notebook. Our prototype measured $190 \mathrm{~mm} \times 140 \mathrm{~mm} \times 1 \mathrm{~mm}$ (Figure 22).

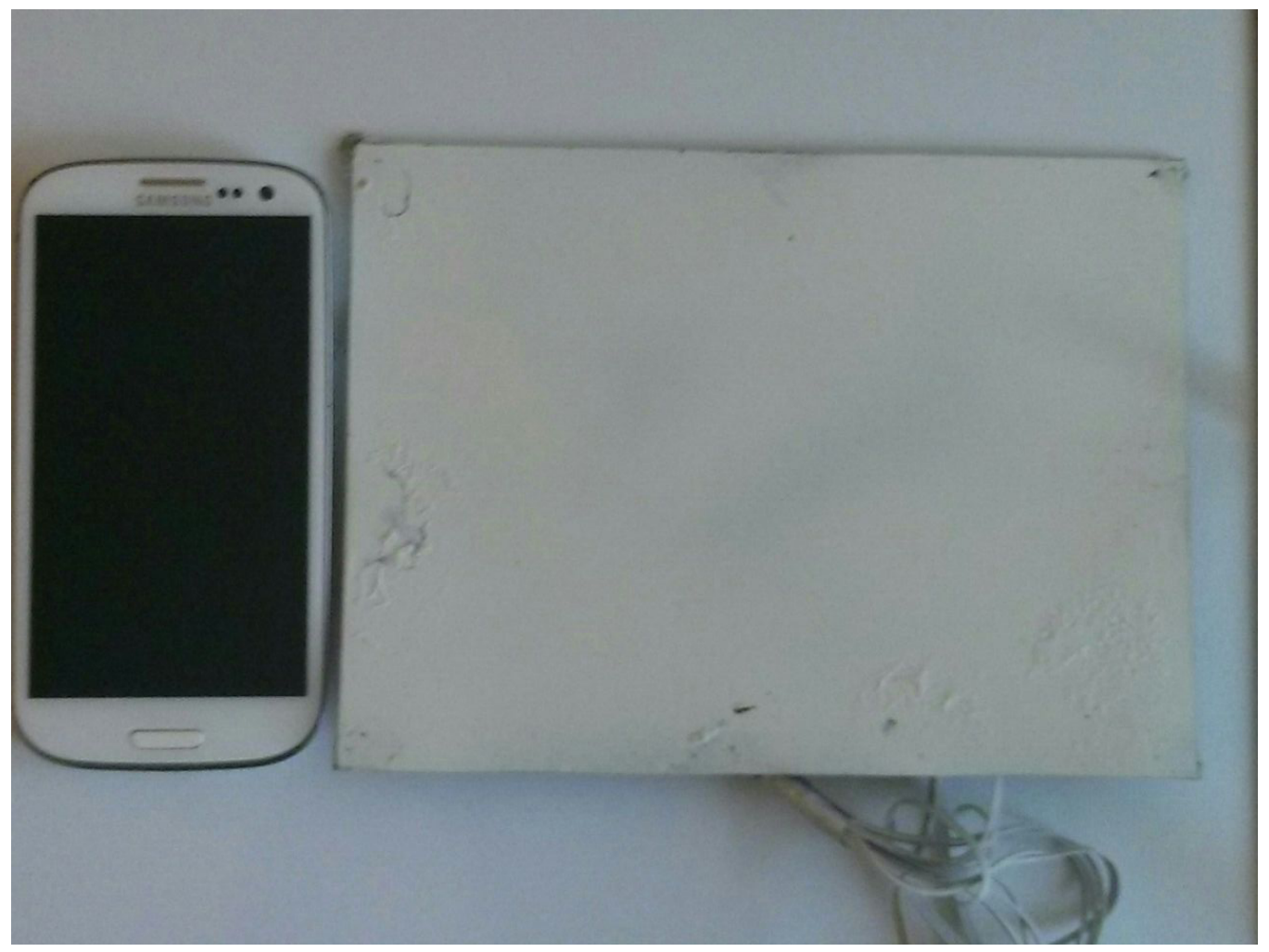

Figure 22: Size of the prototype in comparison with a Samsung Galaxy S3 smartphone 
To start the process of fabrication, we tested several sheets made out of different materials such as polycarbonate, fiberglass and plastic, looking for the suitable weight, flexibility, and simplicity of use. At the end, we decided to use a plastic sheet, which had the flexibility that fits our goals in this research and also it could retain its shape after extended trials. We attached six bidirectional Flex Point sensors [41] to the back of the device (Figure 23). We located one bend sensor in each corner of the device and we attached two bend sensors at the middle, one in vertical direction and one in horizontal direction. In this way, we were able to detect the 12 gestures that we wanted to consider in our experiment. We sprayed the front of the device with a white spray (Figure 23). Then, we connected the prototype to an Arduino microcontroller with thin wires, which transfer the raw data from sensors to the board. Finally we used a Pico projector to show the interface on the prototype and we video recorded the experiment with a Nikon camera.
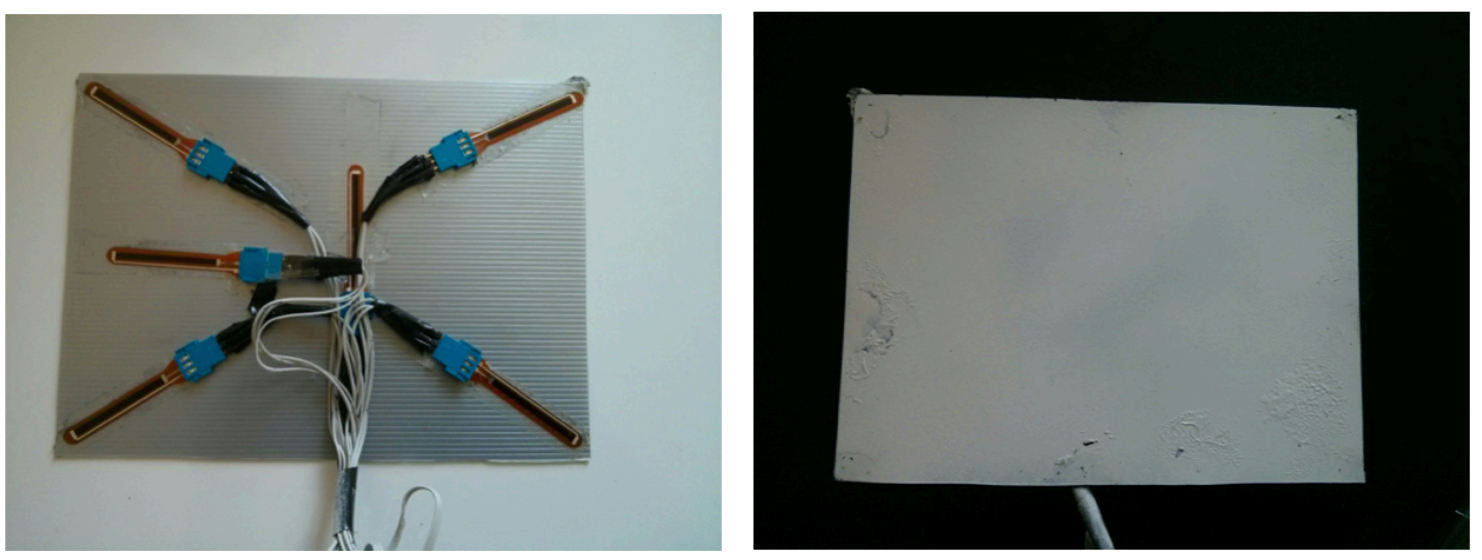

Figure 23: Front and back side of the prototype 


\subsubsection{Software}

The prototype is connected to a MacBook Pro via USB. We wrote an Arduino program to receive and process the raw bend sensor data. We implemented a digital smoothing algorithm to eliminate any outliers from the raw data and then we mapped the smoothed data to a range of 0 to 100 . Activation threshold values were set for the up and down directions. After the prototype is connected to the computer, the program received the neutral state value from the sensors: this is our prototype calibration. The thresholds for full up and down activations were +10 and -10 from the neutral state. Since we also wanted to show the half activation states in our designs, we set the half up and half down activations to be +5 and -5 of the neutral state. To obtain these thresholds, we tested several values to indicate the smallest value that could be consistently recognized for setting the thresholds. Java program was written to first receive the data from Arduino program, second, randomly assign functions to bend gestures, third, show the relevant information about tasks and visual feedbacks on the display and finally fourth, record the quantitative data such as duration of the tasks and number of errors.

The first reasons for randomizing the algorithm of assigning functions to bend gestures was preventing the users from memorizing the gestures during the first trials. If all the gestures and their functions were the same in every trial, the user would not have to notice the designs after a period of working with the device, which could lead to negative effects on our results. The second reason for randomizing the algorithm was avoid guessing or doing the gestures by chance. For example, for moving down in a menu, most of the users can guess they have to bend 
somewhere in the bottom of the device, but this algorithm changes the location and function of the gestures randomly. Hence, the goal here was not to test the mapping between the gestures and actions, only to test the visual designs.

Finally, we must note that the idea behind randomly assigning functions to the gestures is not ecologically valid. We selected to randomly assign functions to gesture to provide a strong experimental environment to measure and compare usability of our visual feedback designs. Our goal was not to provide the best bend gesture to perform each action. In the real life applications, gestures would not be mapped randomly to actions. Most of the previous studies about bend gestures on flexible displays $[9,13,14,19,31,35]$ attempted to find the ecologically valid bend gestures to map gestures to the functions in a way that users could learn, perform or remember gestures better.

\subsection{Experiment design}

We developed an experiment to evaluate the efficiency, advantages, and disadvantages of each proposed design. We developed two tasks in which the user had to perform several bend gestures using different visual feedback designs on our prototype: The first task was image docking, which is an application in which users had to put an image inside a frame. The second task was a simple game in which users had to move a character from a start location to an end location by performing a set of bend gestures.

Our main research goals were to determine an overall preference for visual feedback designs, evaluate the usefulness of different aspects of each design and 
observe behavioral patterns of users during performing each task. We measured tasks completion time and the number of errors that users made during the user study. To gain more insightful information about usability of the designs, we gathered qualitative data using a questionnaire with 5-point Likert scale questions about usefulness of each design on showing different information such as direction, location, angle, wrong bends and half/full activation. The questionnaire was administered after the participant finished each task with each design. In order to determine user's overall experience, after the participant finished the entire tasks, they were asked to fill out another questionnaire about their overall experience and compare designs in general.

Each session was approximately an hour in length. Prior to starting the experiment, participants were required to complete a warm-up tutorial, designed to teach them how to correctly perform the gestures and how to read the information of each feedback designs. The users compensated with a $\$ 10$ gift card for their participation.

Figure 24 shows the experiment setup with a participant performing a bend gesture. 


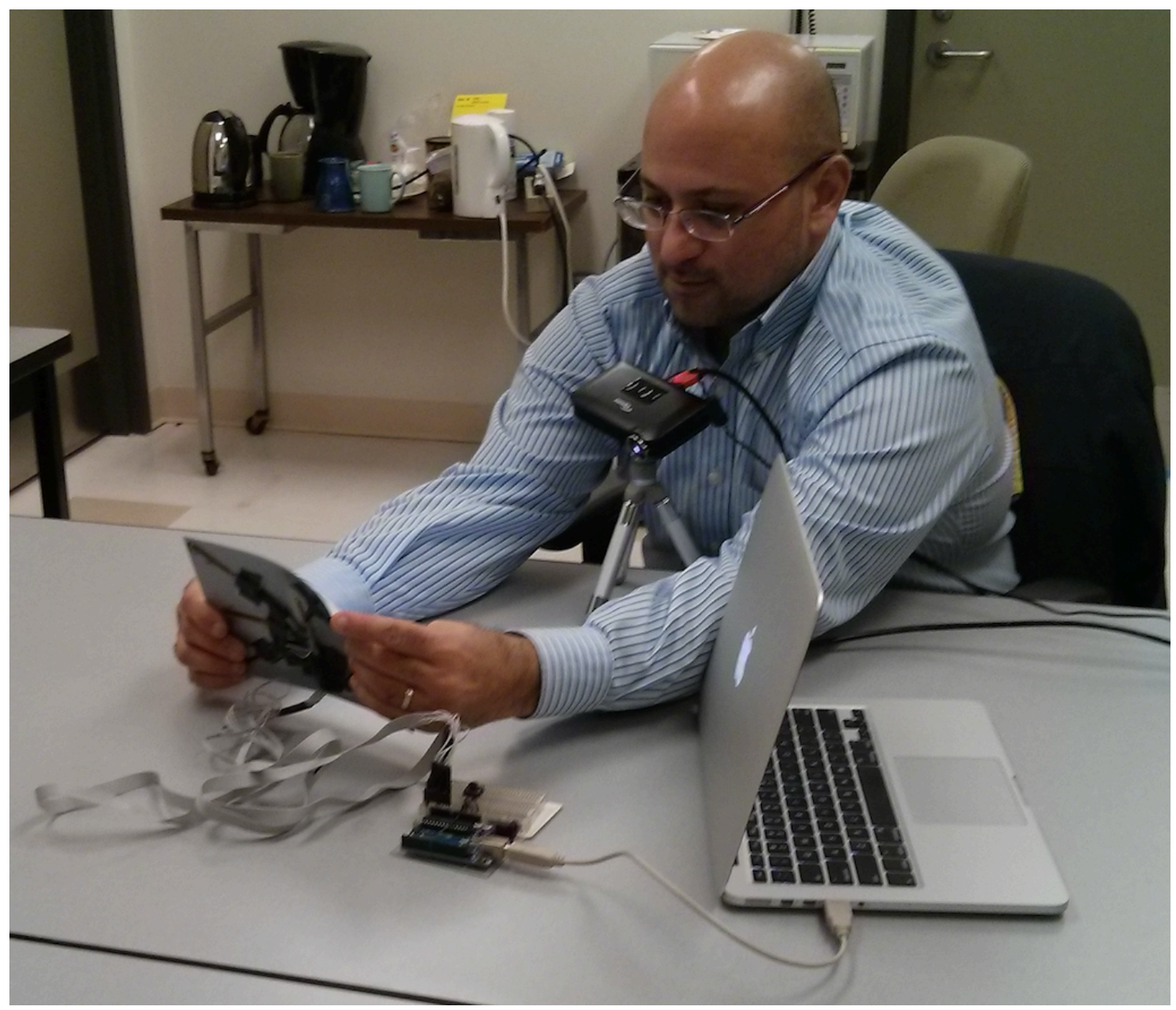

Figure 24: A Participant at the middle of an experiment

To compare the influence of the feedforward mechanism and feedback mechanism in central circle design and arrows design separately, each task had to be performed twice using each design, once with feedback and once without feedback. In this way we were able to compare the result of each condition (with or without feedback) to gain more information about each mechanism in this concept. In addition, for gaining more information about each feedback design in particular and to have a better comparison between each feedback design, cheat sheet design had to be performed three times: 1) With central circle feedback 2) With arrows feedback and 3) Without feedback. Participants were asked to perform our first 
task's trials, with all of the mentioned conditions, twice, and asked to perform our second task's trials with each condition only once to avoid a long experiment session.

In summary, the total number of trials performed by each participant was as follows (Figure 25):
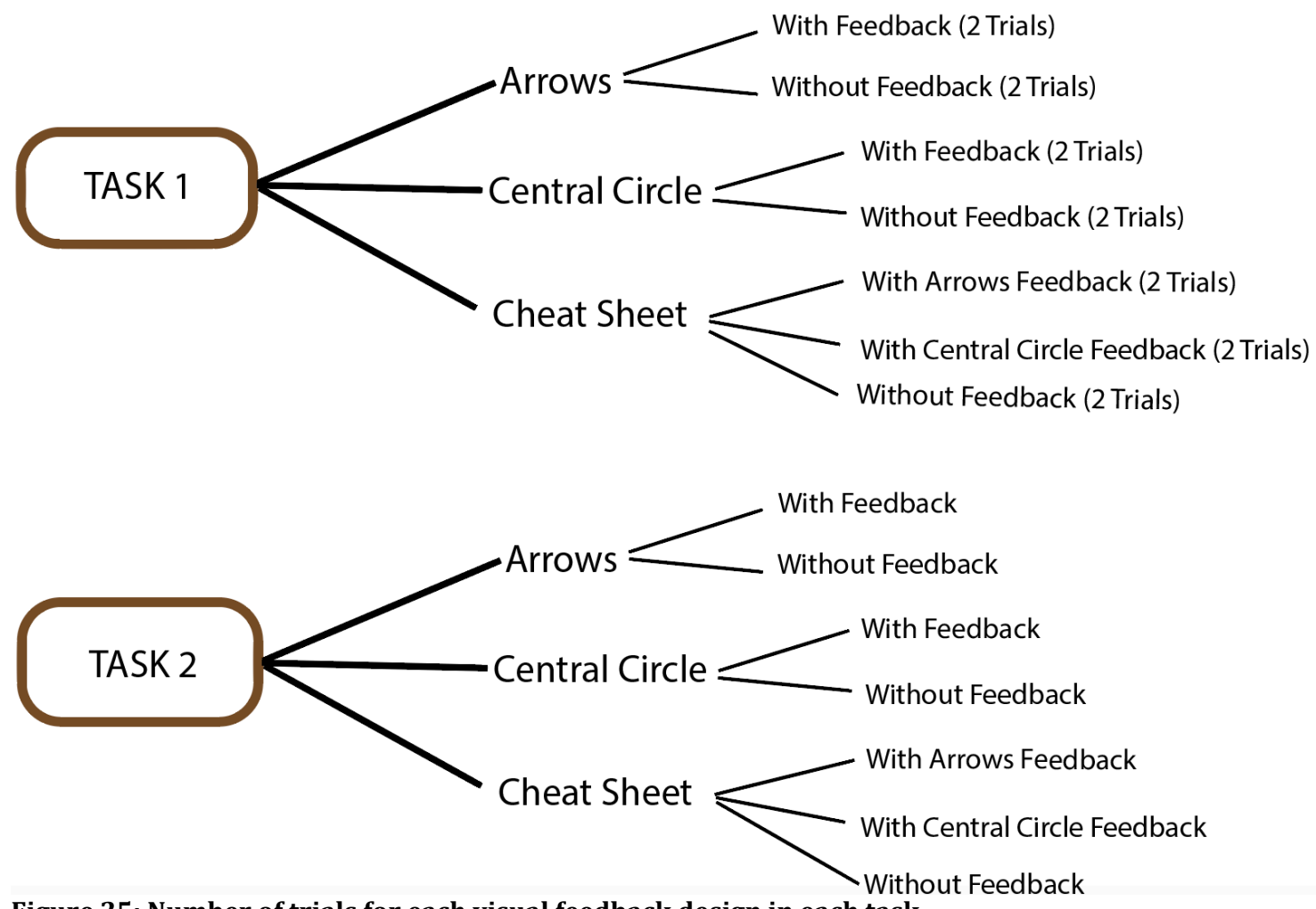

Figure 25: Number of trials for each visual feedback design in each task

\subsection{Task 1: Image Docking}

We implemented a photo manipulation (image docking) application, based on a study by Kildal et al. [15]. The task was to use bend gestures with different visual feedback techniques to put a photo inside a frame by moving it up and down, right and left, and scaling it (Figure 26). The aim of this task was to put the image inside the frame as quickly as possible with the least amount of error. In the similar 
task in Kildal et al. study they asked participants to fit the image to a frame with a $5 \%$ error rate. We decided to take a different approach and made our first task easier by asking participants to only put the image inside a frame, with no requirement for the image and frame to be the same size. Consequently, in this task, as long as the picture was completely inside the frame, the task was done. We made this design decision because in fabrication of our prototype, we used projection instead of a real display and this could result in difficulties in performing tasks with much accuracy.

The user could move and scale the image by performing the correct gestures. There were 6 movements in this task: up, down, right, left, +size, and -size. In each trial, 6 gestures were randomly selected among the 12 possible gestures, and distributed among the 6 movements. The remaining gestures became unavailable.

The user interface contained a black photo frame and a photo shown on top of a grey background. We randomly defined the initial position and size of the photo in a way that should always fulfill the following conditions, 1 . The center of the image was outside the target frame 2 . The image was scaled up to a minimum 1.5 times the size of the frame. 

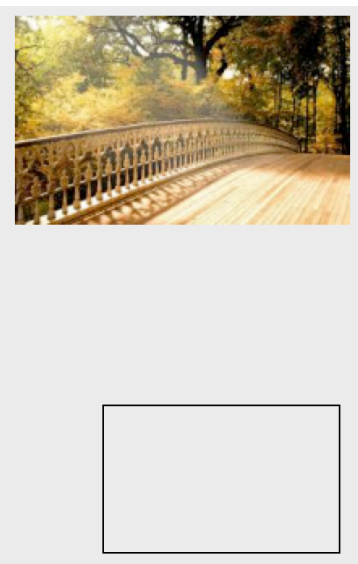

Figure 26: Task 1 (Image docking)

\subsection{Task 2: Game}

We implemented a simple game in which user had to move a character from the origin to the target block in a $4 \times 4$ board, and, along the way, eat all the randomly distributed apples and oranges (Figure 27). The origin block and target block locations were randomly selected in two opposite corner in each trial. For instance, if, in a trial, the origin block was the top right corner, the location of the goal block was the bottom left corner with darker color. It must be noted that eating all the apples and oranges was mandatory for finishing the task and if a user reached to the final grey block without eating all the apples/oranges, he/she had to move back and eat those remaining. 


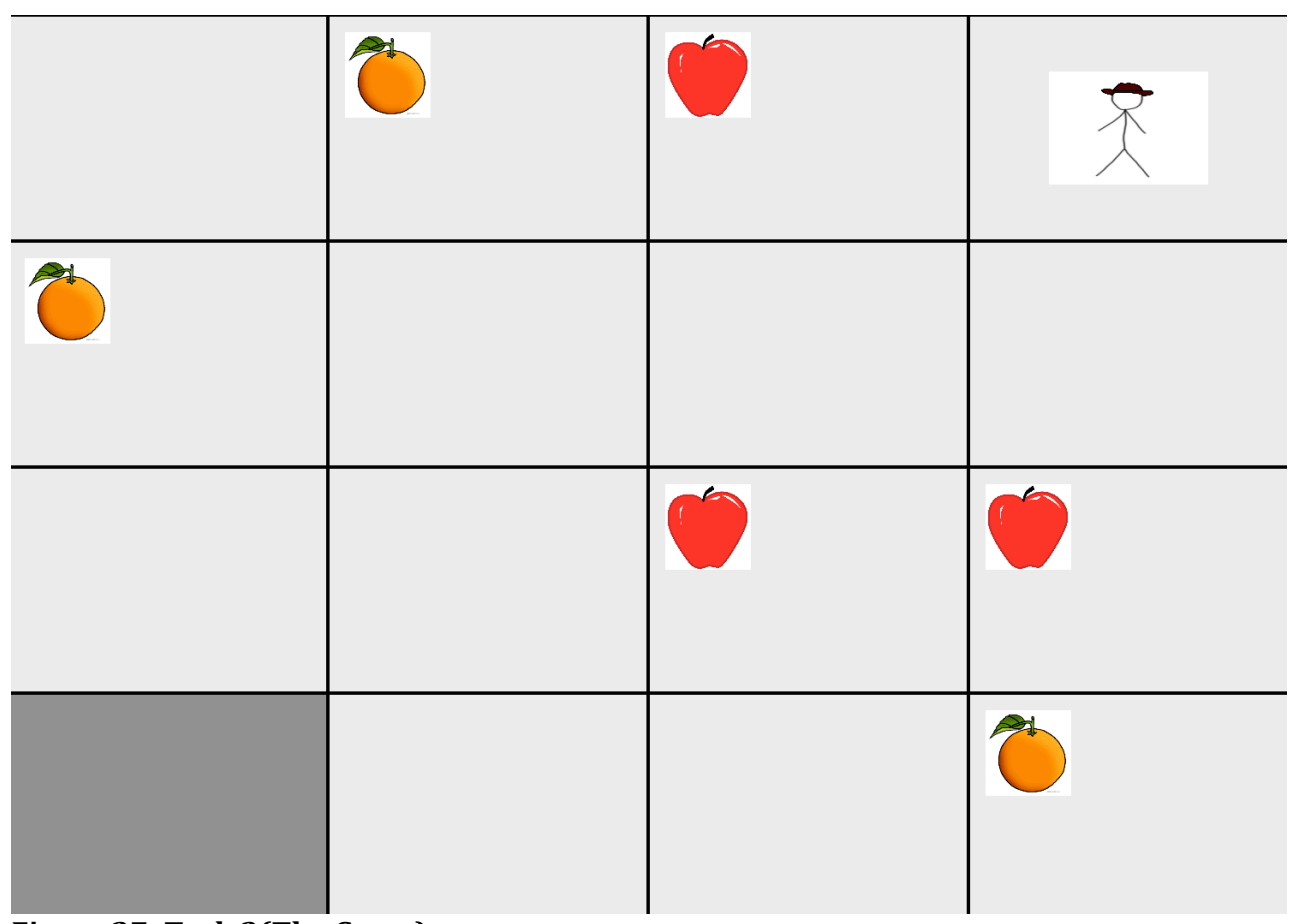

Figure 27: Task 2(The Game)

In this task, the character moves one block at a time. This means that, for each movement, the user had to bend a gesture and activate it, then deactivate and then again start a new bend. This is the main difference between this task and the image docking task, which let the user maintain the gesture to perform a repeated action.

In this task, there were 4 movements (up, down, right, left); and an "eat apple, eat orange" function which, in total, resulted in 6 bend gestures; the rest of the gestures were unavailable.

To complete the game, users had to perform the available bend gestures, which were logical in regards of character position as well. For example, performing an eat apple function in a block without apple in it were recorded as an error. As a 
result in addition to having unavailable gestures as an error, performing incorrect bend gestures in relation to position of the character were also recorded as an error.

\subsection{Hypotheses}

We hypothesized that in both the image docking task and the game task, both central circle design and arrows design are the more efficient techniques (yielding the best completion times and fewer errors) in comparison with cheat sheet design (H1). In these two conditions users have the designed feedforward to show them location, direction and the result of each gesture and also they have feedback to indicate the wrong performed bends and/or angle of the bends. For the same reasons, we also hypothesized that we would observe a higher rating for these two designs in the questionnaires asking about usefulness of each visual feedback design (H2).

We hypothesized that, in general for all three designs (central circle, arrows and cheat sheet), providing feedback would decrease task completion time and error rate because it gives users more information about bend gesture rather than providing only feedforward without any feedback (H3).

Finally, we predict similar performance with the two main designs, as we believed that both of the designs have proper factors for guiding the users.

\subsection{Participants}

Twenty-four participants (11 women) were selected to take part in the experiment. They were on average 24.0 year of age, with a range between 18 and 50 
years old. Among these 24 participants, 3 were left-handed, and 3 of them had worked with a flexible prototype before on previous research on deformable devices. They all used their cellphones regularly. 


\section{Results}

\subsection{Quantitative}

To compare the duration and the number of errors in each trial, we performed one-way Analysis of variance test.

\subsubsection{Image Docking}

We observed a significant difference in relation to time $(\mathrm{F}(6,329)=7.384, \mathrm{p}$ $<0.05$ ) for the image docking task. Figure 28 shows the duration of each condition for task one. We used the Tukey test to investigate pairwise comparisons. 


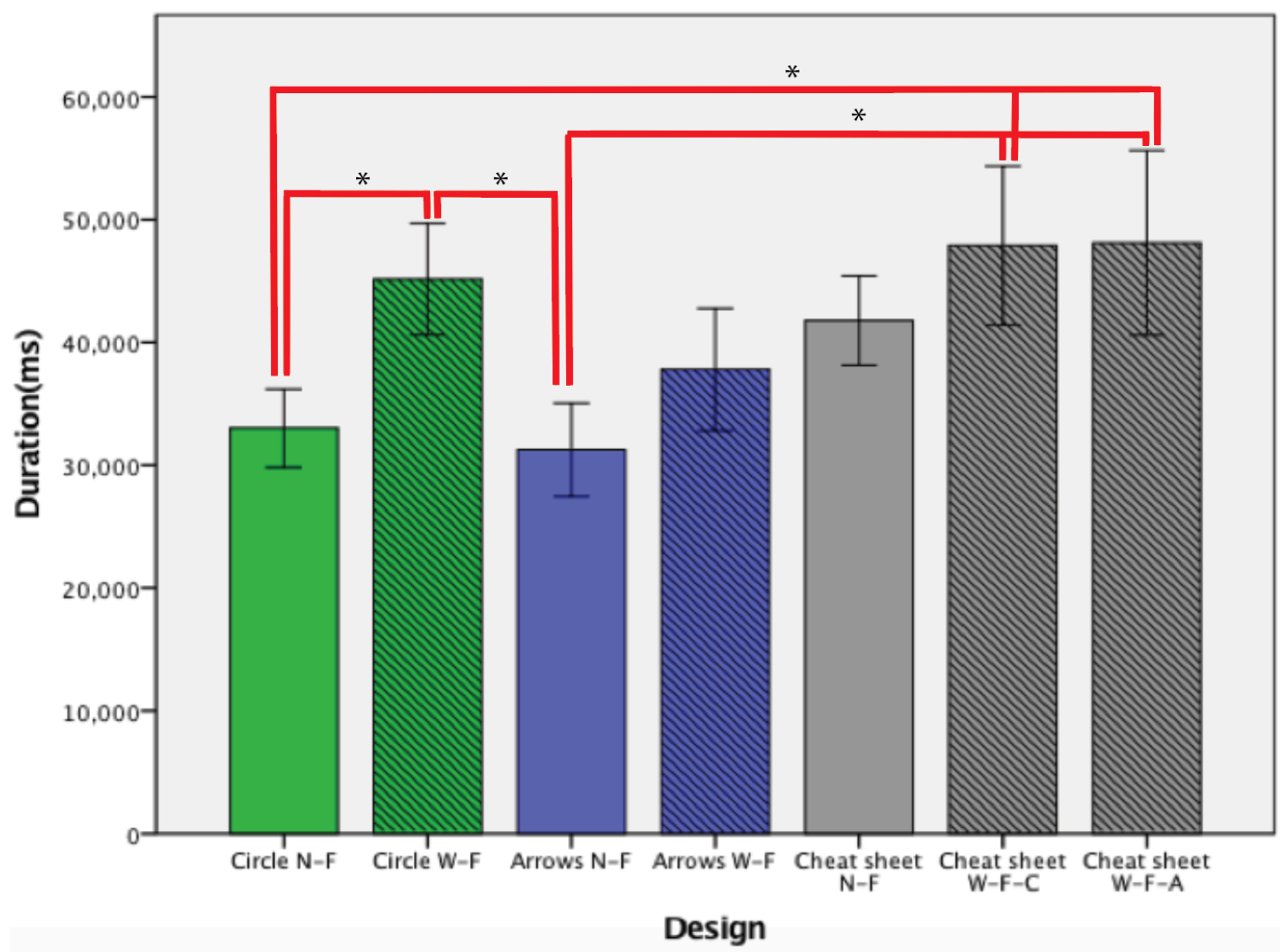

Figure 28: Completion time of image docking task in each design. (N-F= No Feedback, $W-F=W$ ith Feedback, $\mathrm{W}$-F-C = with central circle feedback, $\mathrm{W}-\mathrm{F}-\mathrm{A}=$ with arrows feedback). Error bars represent confidence interval

We found a significant difference between the central circle design with feedback $(M=45153 \mathrm{~ms}, \mathrm{SD}=15639)$ and without feedback $(\mathrm{M}=33020 \mathrm{~ms}, \mathrm{SD}=$ 11011). On average, the users performed task one by using the central circle design without feedback $26 \%$ faster than they did by using the central circle design with feedback. While we also observe a similar trend of a slightly faster performance without feedback then with in the other designs, in the arrows design $(17 \%$ faster without feedback in the arrows design, and $12 \%$ faster without feedback in the cheat sheet design), we did not find the difference to be significant. 
We found the arrows design without feedback to be the fastest, and significantly different than the central circle design with feedback $(F(1,94)=$ $22.219, \mathrm{p}<0.05)$, the cheat sheet design with circle feedback $(\mathrm{F}(1,94)=19.924, \mathrm{p}<$ $0.05)$, and the cheat sheet design with arrow feedback $(F(1,94)=18.149, p<0.05)$.

We also found central circle design without feedback to be significantly faster than the cheat sheet design with circle feedback $(F(1,94)=18.654, \mathrm{p}<0.05)$, and the cheat sheet design with arrow feedback $(F(1,94)=17.026, p<0.05)$.

We note that the time difference between the cheat sheet design with circle feedback and the cheat sheet design with arrow feedback is negligible, and not significant $(F(1,94)=0.02, p>0.05)$. As the feedforward of the cheat sheet design was a basic table and it was same for both cheat sheet design with arrows feedback and cheat sheet design with central circle feedback, we hypothesized that any differences between the cheat sheet design with arrows feedback and cheat sheet design with central circle feedback was generated from the differences of the feedback mechanisms. This negligible time difference indicates that there is no significant difference between these two feedback in helping users to do task one more quickly.

We performed another one-way Analysis of variance test for number of errors in image docking task. We did not observe any significant differences. The bar chart below shows the number of errors in each condition for image docking task (Figure 29). It is noticeable that the number of errors in cheat sheet design is slightly higher than other designs. We hypothesized that these differences are the 
result of using texts to show directions and locations and especially the words

"horizontal" and "vertical" might generate difficulties for users.

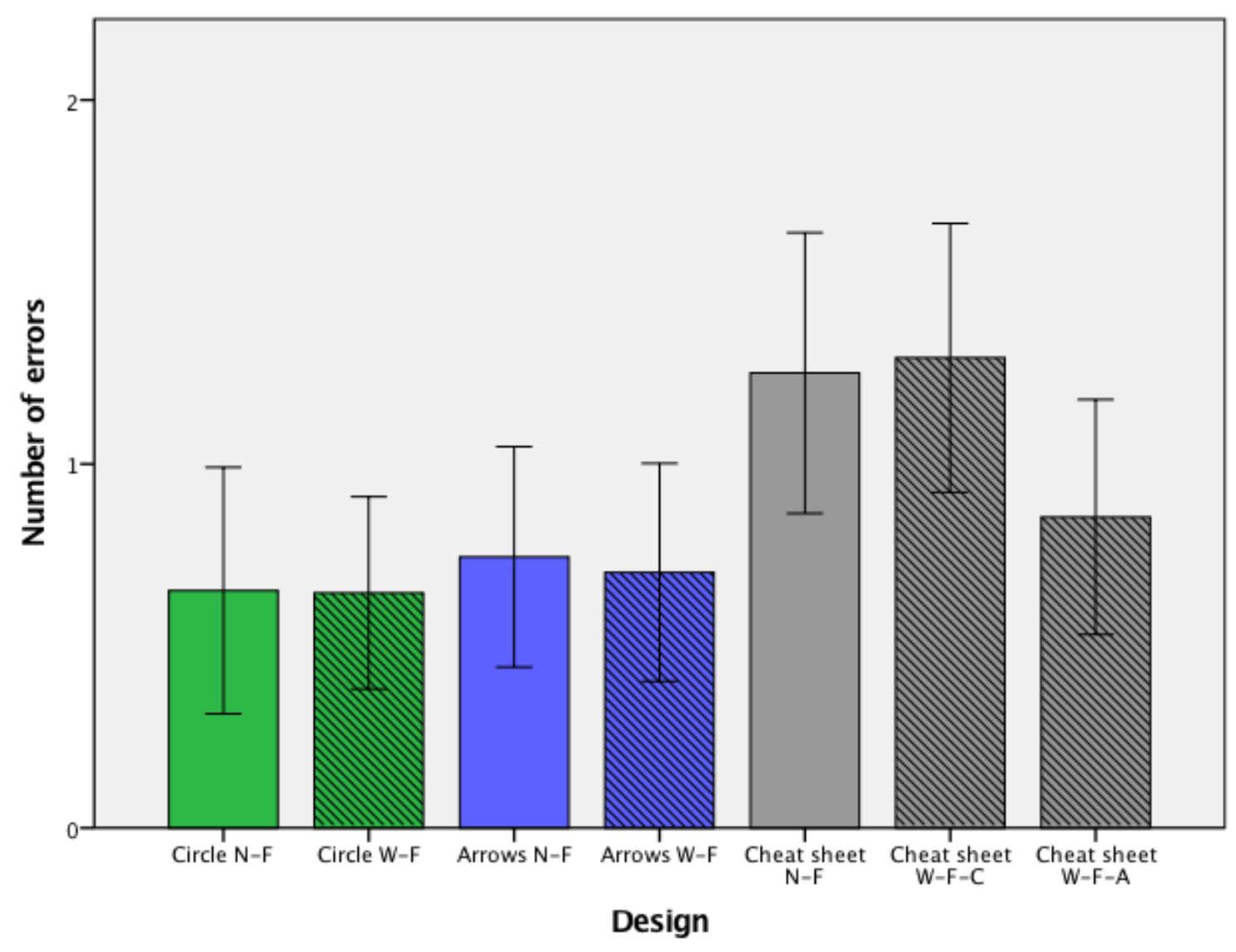

Figure 29: Number of errors during performing image docking task with each design (N-F= No Feedback, W-F = With Feedback, W-F-C= with central circle feedback, W-F-A= with arrows feedback). Error bars represent confidence interval

\subsubsection{The Game}

We observed a significant difference between the times of operation (F $(6,161)=3.771, p<0.05)$ for the game task. We investigated the pairwise comparisons by performing a Tukey test. In this task, users were significantly faster with the arrows design without feedback than with the cheat sheet design without feedback $(F(1,46)=14.682, p<0.05)$, and the cheat sheet design with arrows 
feedback $(F=(1,46)=6.012, p<0.05)$. Figure 30 shows the average duration for each of the designs.

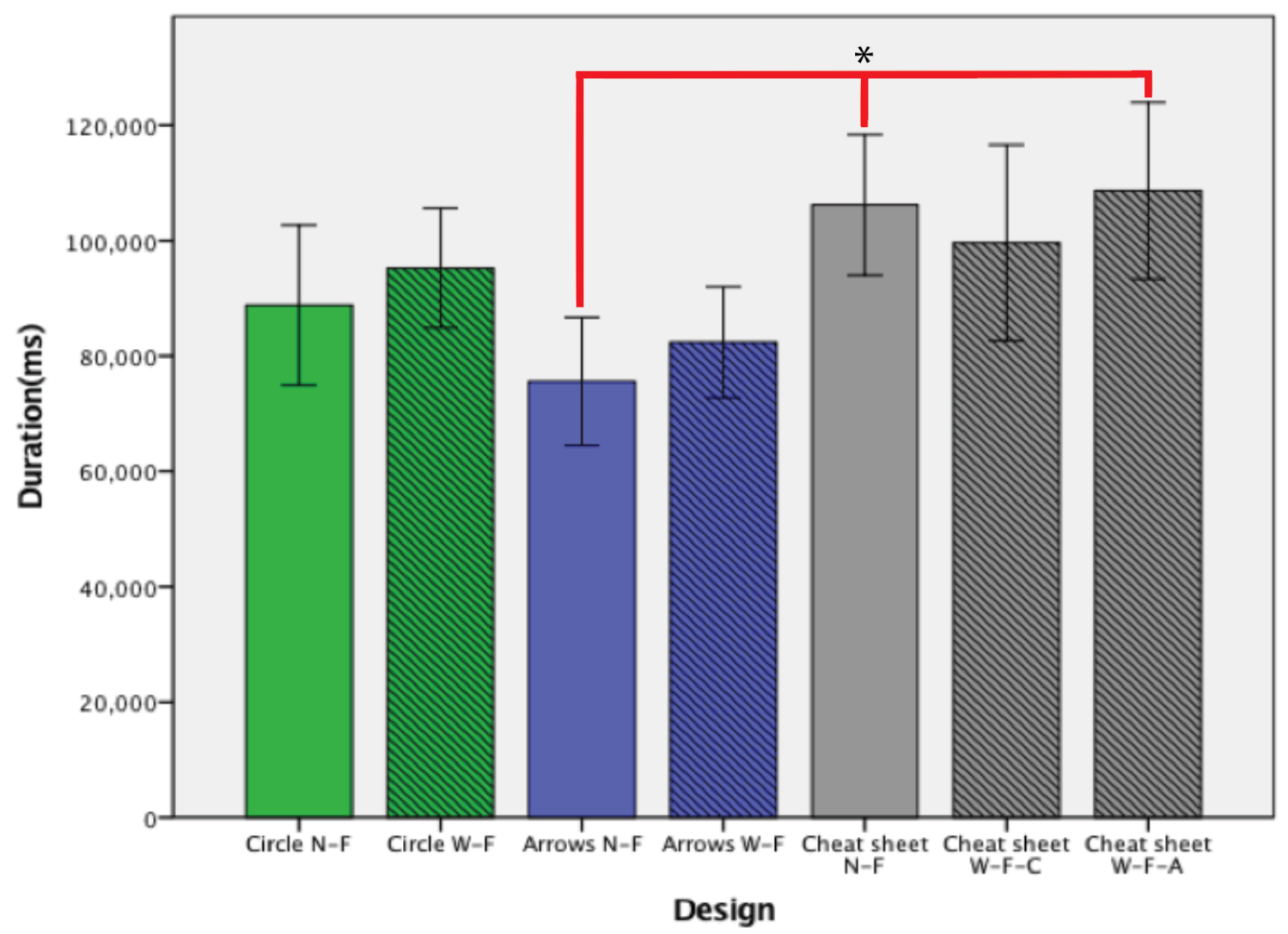

Figure 30: Completion time of the game using each design. (N-F= No Feedback, W-F= With Feedback, WF-C= with central circle feedback, $\mathrm{W}$-F-A= with arrows feedback). Error bars represent confidence interval

Again, the best performance in regards to time was for the arrows design without feedback $(M=75557 \mathrm{~ms}, \mathrm{SD}=26360)$. Similar to the image docking task, overall the users had better performance without the application of feedback in central circle design and feedback design. However, the differences were not significant. 
When comparing the number of errors, our one-way ANOVA found no significant differences between the cases. Though not significant, it is interesting to note that in all three designs, the users had fewer errors when they were not provided with feedback. The bar chart below shows the number of errors in each case (Figure 31).

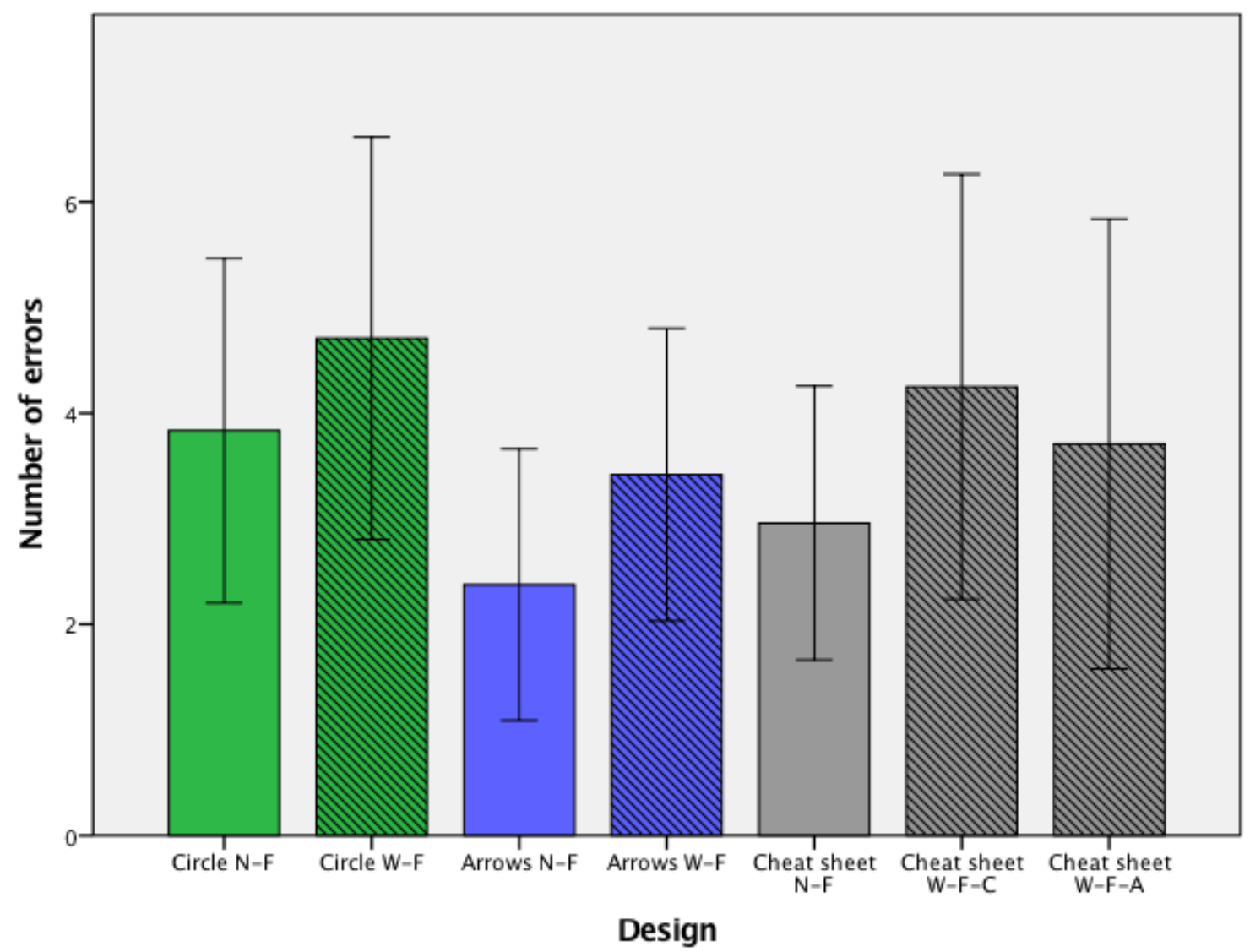

Figure 31: Number of errors during performing the game in each design. $(\mathrm{N}-\mathrm{F}=\mathrm{No}$ Feedback, $\mathrm{W}-\mathrm{F}=\mathrm{With}$ Feedback, $\mathrm{W}-\mathrm{F}-\mathrm{C}=$ with central circle feedback, $\mathrm{W}-\mathrm{F}-\mathrm{A}=$ with arrows feedback). Error bars represent confidence interval

\subsection{Qualitative data}

We applied the Friedman test to analyze the Likert questions in our questionnaire. 


\subsubsection{Image Docking}

When asked about efficiency of each design in indicating the gestures locations, the users found the arrows design to be significantly better than the others $\left(\mathrm{x}^{2}=7.467, \mathrm{p}<0.05\right)$. We asked about efficiency of each design in indicating the gesture direction, the arrows design was also significantly better that the others $\left(x^{2}=6.562, p<0.05\right)$. The central circle design arrived second. However, in the question about the distracting properties of each design, the users found the arrows design to be significantly less distractive $\left(x^{2}=7.345, p<0.05\right)$. We found a significant difference between the central circles design with feedback and the central circles design without feedback $\left(x^{2}=5.937, p<0.05\right)$, meaning that when the feedback was activated in the central circle design, the users found it far more distracting than the method without feedback. Finally, in regards to the question on the clarity of the feedbacks in showing the errors, we did not find any significant difference in users responses.

\subsubsection{The Game}

We found similar data to the data in the previous task. The users found the arrows design to be significantly better in indication of location $\left(x^{2}=6.267, p<0.05\right)$ and direction $\left(x^{2}=6.767, p<0.05\right)$. But we did not find any significant difference in the answers to questions about the designs' distraction features. The users also found no difference among the designs in showing the errors; the comparison yielded no significant differences. 


\subsubsection{Final Questionnaire:}

In the final questionnaire about user's overall experience with each design, $83 \%$ of the users expressed that arrows feedforward was the most preferred feedforward design. For the second rank, meaning the second preferred feedforward design, $70.5 \%$ of the users selected central circles, and arrows and cheat sheet were in the next places with $17 \%$ and $12.5 \%$, respectively. For the third rank, meaning the least favorite feedforward design, $82.5 \%$ of the users found the cheat sheet design as the least popular one, $17.5 \%$ declared it to be central circle, and interestingly, no user select arrows as the least preferred feedforward design. Figure 32 shows the percentage of user's rating for each feedforward design.

$$
\text { Arrows Design } \square \text { Central Circle Design } \square \text { Cheat Sheet Design }
$$

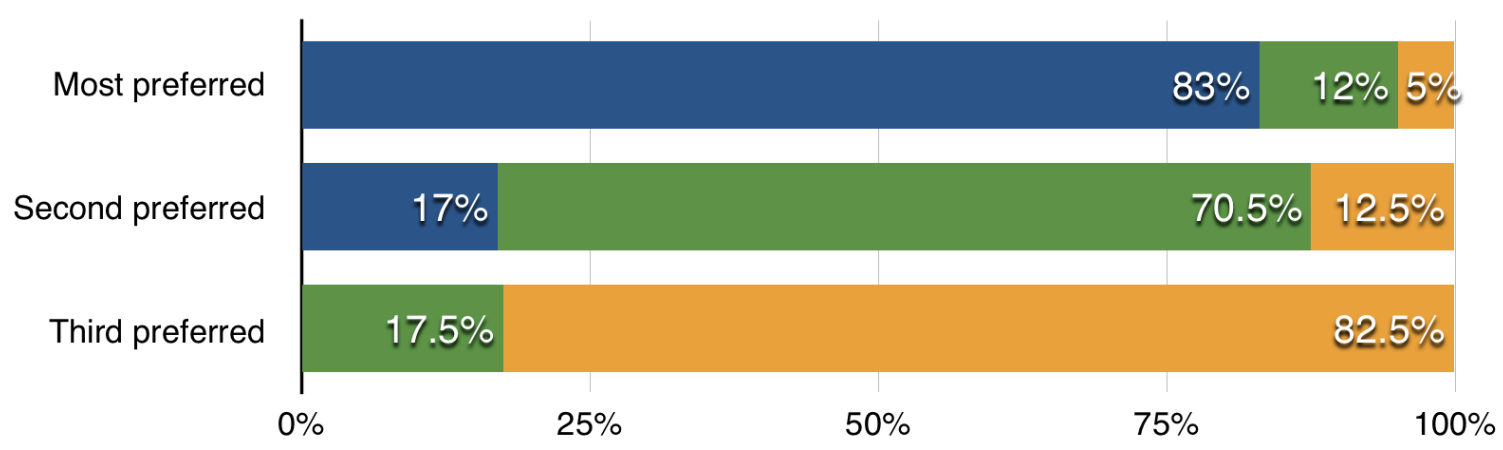

Figure 32: Level of user's preference for each feedforward design

In regards to the question that asked the user to rank the visual appeal of the designs, $50.5 \%$ of the users put the central circles design in first place, $32.9 \%$ found the arrow design as the best design, and only $16.6 \%$ of the users put the cheat sheet 
design in the first rank. For the second rank, $49.5 \%$ chose central circle design, $37.5 \%$ declared it to be arrows design, and $13 \%$ found cheat sheet to rank second. Finally, for the third rank, $70.4 \%$ put the cheat sheet design, $29.6 \%$ chose arrows design, and no one found central circle design to be the third-ranking design. Figure 33 shows the users ranking for visual appeal in each design.

Arrows Design $\square$ Central Circle Design $\square$ Cheat Sheet Design

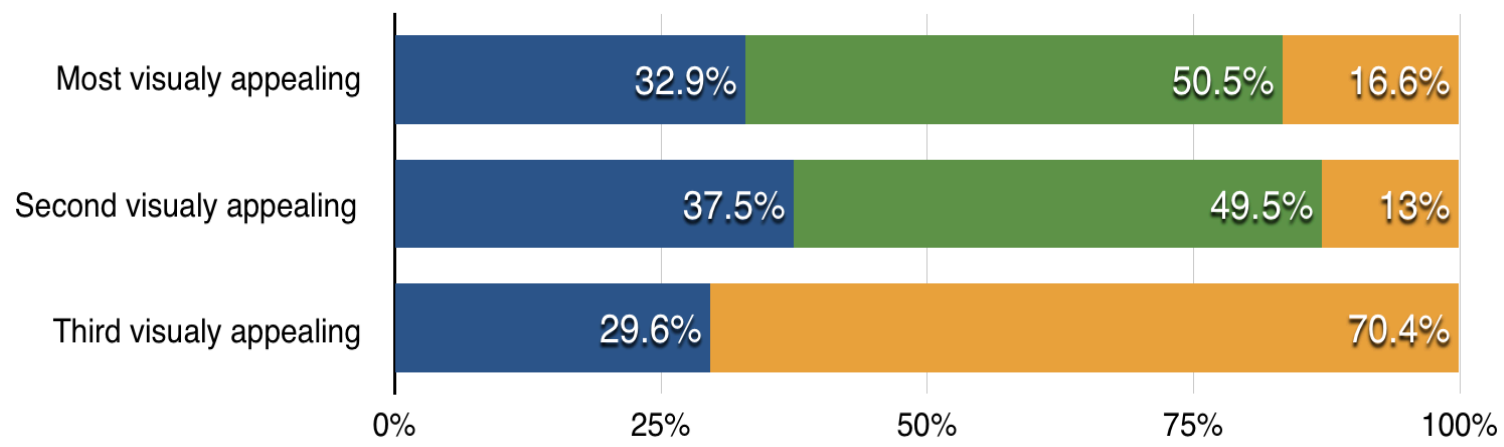

Figure 33: Designs' ranks in terms of visually appealing

The next question asked the user to classify the designs in terms of having useful information: $58.3 \%$ selected arrows design, $20.9 \%$ chose cheat sheet design, and $20.8 \%$ put central circle design in the first rank. For the second rank, the $45.8 \%$ selected central circle design, arrows designs had $37.5 \%$ of the votes, and cheat sheet design had $16.7 \%$ of the votes. Finally, for the third rank, $62.5 \%$ voted for cheat sheet design, $33.3 \%$ voted for central circles, and $4.2 \%$ selected arrows design for the third rank. The chart below shows the rank of each design for useful information (Figure 34) 


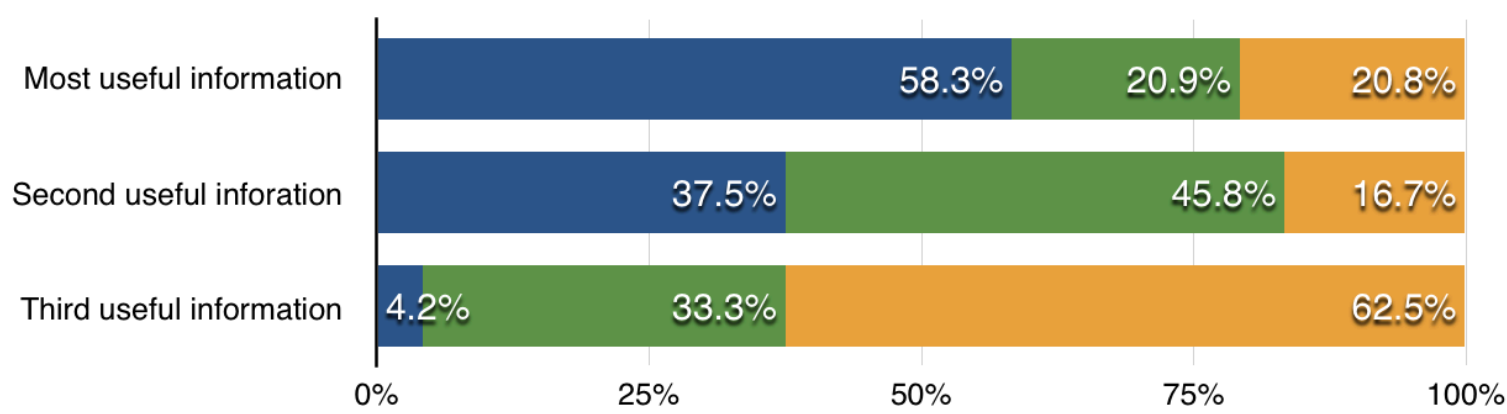

Figure 34: Designs' ranks in terms of containing useful information

Among the two types of available feedback in the central circle design and arrows design, regardless of feedforward, $54.2 \%$ found arrows design to be the most interesting, and 37.5\% preferred central circles. A Wilcoxon Signed-Rank test was run to determine if there was a significant difference between users' rating for arrows feedback and central circle feedback. There was no significant difference between users' ratings for arrows feedback and central circle design. But in the question that only asked the users' opinions about the feedbacks in regards to showing errors and incorrect bends, $28.7 \%$ preferred the red arrow in arrows design, and $58.8 \%$ preferred the red cross sign in the middle of the display in the central circle design. Only $12.5 \%$ preferred a no-feedback state. We ran a second Wilcoxon Signed-Rank test to determine if there was a significant difference between users' ratings for central circle design error feedback and arrows design's error feedback. We found that users' significantly preferred central circle design's error feedback better than arrows design error feedback $(\mathrm{z}=.008, \mathrm{p}<0.05)$. 


\section{Discussion}

In our research on the methods of guiding users to perform bend gestures on a flexible display, we found insightful data about the effect of each of our proposed visual feedback designs on participants' performance. In this section, we take a deeper look at our results and discuss our main findings.

Overall, our results supported our first hypothesis about arrows design and central circle design improving users' performance in both tasks (H1). Participants performed better using these two designs, in comparison with cheat sheet design. Additionally, our results showed that users performed tasks faster with the arrows design without feedback in both tasks. We also found that most users (83\%) chose the arrows design as their preferred design. The central circle design came in second place (12\%), and the cheat sheet design was the least preferred (5\%). These results support our second hypothesis, and participants' ratings were higher for arrows and central circle design (H2). Furthermore, our quantitative results do not support our hypothesis about the usefulness of the feedback design, and we observed that giving feedback deteriorated performance and did not lead to more beneficial results (H3).

We have organized our discussion chapter into the following parts: (1) design comparison, (2) effect of each design on user performance in task 1 and task 2, (3) feedback, (4) design recommendations and (5) limitations. 


\subsection{Design comparison}

We began our design comparison by comparing the arrows design with the central circle design in the first subchapter. In the second subchapter we compared the elements of arrows design and central circle design with cheat sheet design.

\subsubsection{Comparing the arrows design with the central circle design}

The arrows design and central circle design each had strengths and weaknesses. For example, the central circle design was more visually appealing than the arrows design according to participants' ratings, while the arrows design was more informative. Overall, the arrows design proved to be better than the central circle design in terms of both quantitative and qualitative data.

Although the time differences between the arrows design and the central circle design were significant only in one situation (users performed task 1 with the arrows design without feedback significantly faster than the central circle design with feedback), it is worth mentioning that, in all the other situations, performance times of the arrows design were slightly faster than the central circle design.

Our result showed that $75 \%$ of participants found that it was much easier to see the information and perform a bend gesture at the same time when the information appeared at the actual location of the bend gesture, which was the case in the arrows design. As one user commented on feedforward, "in the arrows, the arrows appear in the exact place of bend, and it simplifies the user's task." However, the other $25 \%$ preferred to see all the information at one area of the display, which was the case in the central circle design. In this way, they always knew where to find the information. 
Twenty-eight percent of participants made comments about the size of each design. According to these participants, the fact that the arrows design occupied a smaller area of the screen than the central circle design had a positive effect on users' performance. The arrows design occupied approximately $14 \%$ of the display, whereas the central circle design occupied around 30\%.

In our observations, we found one drawback to showing the information at the same location as the bend gesture. As participants were performing bend gestures, a certain amount of the information (such as shapes or text) became obscured, because (1) the participants' hands were blocking the view of the users, and (2) performing a bend gesture resulted in a curve in the screen (Figure 35). The central circle design did not present the same problem, because the information appeared in the middle of the screen and was always visible. To overcome these problems, one alternative is moving the graphical shapes and text of the design to a location where the user can easily see them.

In addition, according to users' responses on the questionnaire, the central circle design was more visually appealing than the arrows design. This finding is interesting; although the arrows design was more helpful to users, the circle design was their top choice for a tool that was "well designed." 

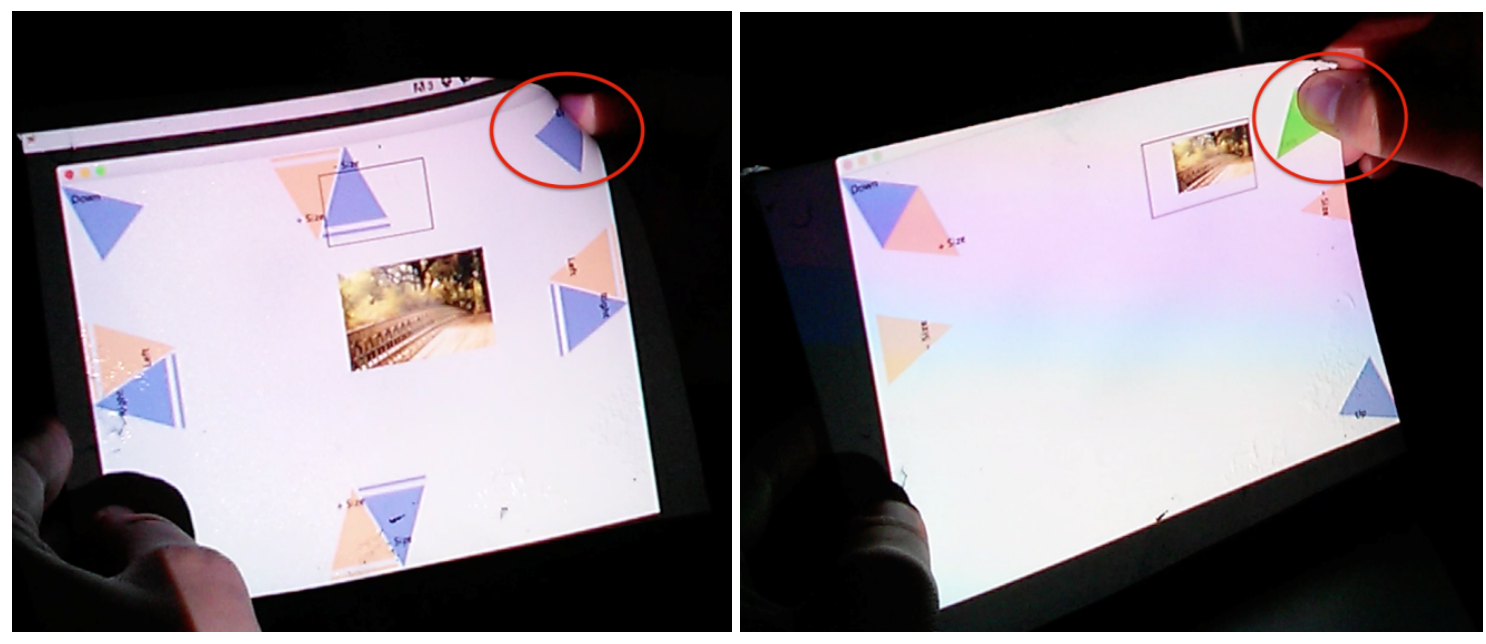

Figure 35: Information becoming obscured in arrows design.

An interesting comment we received was that if we used a rectangular shape instead of a circular shape in the central circle design (because the display is rectangular), it could better help users (Figure 36). This could result in a better understanding of each arrow and shape's relation to each corner with regard to the direction of the display.

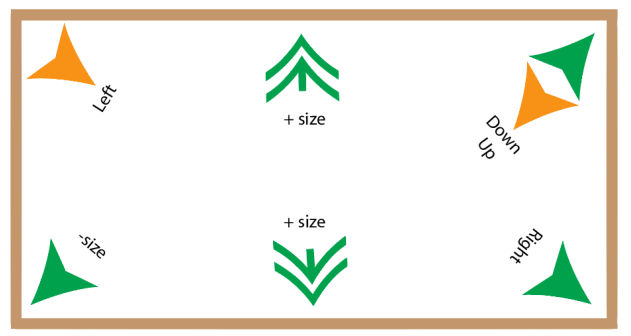

Figure 36: Using rectangle instead of circle 


\subsubsection{Comparing the Arrows and Central Circle Designs with the Cheat Sheet Design}

As we hypothesized, the users' performance with the arrows designs and the central circle design was better in terms of time needed to finish both tasks and users' ratings. However, they were not noticeable in terms of number of errors. During the experiment, we observed that most users had problems recognizing the horizontal and vertical bend gestures. When using the cheat sheet design the same users did not have that problem using the central circle and arrows designs. These two designs were also better at indicating the dual bend gestures, and they reduced the problems related to dual bend gestures significantly.

One of the main advantages of the cheat sheet design was that it took up only $12 \%$ of the display; as a result, no user complained about it covering actual information of the tasks. Additionally, this design is similar to the central circle design in that it always appeared in a fixed location on the screen, which was a positive feature for the same participants who listed the central circle design's fixed location as an advantage.

In the central circle and cheat sheet designs, users had to look at one location of the display to gain information and then perform the bend in another location, i.e., they had to look at two places to perform one bend. According to some of users' comments, this might have had a negative impact on users' performance. On the other hand, one user mentioned, "one of the best features of this design is that because the place of the circle is always fixed, the user knows where to look to find the information." This comment indicates that the fixed shapes, despite their 
negative aspects, could also have some positive aspects that may be useful in some applications.

\subsection{Effect of the visual feedback designs during each task}

In this subsection, we discuss the effect of visual feedback designs on users' performance in regard to the interfaces of the two tasks.

Task 1. To correctly place the image inside the frame in task 1 , the user had to perform several small movements with bend gestures, which required a lot of attention. The participants performed faster when using the arrows design during the image-docking task. This shows that the arrow design can be useful for applications that require much of the user's attention, and in which accuracy of bend gestures is important. Performing the details of a complex password for flexible displays which require several small and accurate bend gestures can be an example of such applications; these designs can also be helpful for applications that require zooming in and out while taking a picture.

Task 2. The user interface for this task contained 16 blocks, 3 apples, 3 oranges, and a character. Its many elements made it a more realistic interface then the first task. Like task 1, users had the fastest interaction with the arrows design, followed by the central circle design; but the difference was mostly insignificant. These results show that these designs are appropriate for applications, such as games, that contain busy interfaces. However, further research is required to confirm this.

Overall, given the fact that both the arrows design and the central circle design resulted in a better performance than the cheat sheet design on both tasks, 
we expect that our visual feedback design concepts can be used in other applications with different interfaces.

\subsection{Feedback}

We divided this subsection into two parts: 1 . Feedback for correct bend gestures and 2. Feedback for wrong bend gestures.

\subsubsection{Feedback for correct bend gestures}

Contrary to our hypothesis that having feedback on the designs would result in faster task completion with fewer errors, we observed that having feedback on all designs, on average, not only had no positive effect on user performance in terms of time and error rates, but also increased the time needed to complete both tasks. Surprisingly, in task 1 , the difference in completion time, using central circle design with and without feedback was significant. In reality, feedback distracted users instead of helping them perform their tasks better. However, the results of our questionnaires showed that the majority of the participants (66\%) acknowledged the potential usefulness of the feedback design. This result suggests that, while feedback had a negative effect on users' performance, it had a positive effect on users' confidence that they were performing the correct bend gesture.

As we mentioned before, when users did not have feedback on the central circle design, they performed the image-docking task significantly faster than they did when they had feedback. One of the reasons for this could be that, in this design, the feedback appeared in the middle of the screen and was a large, green circle. Thus, it may have caused some amount of distraction (Figure 37). However, because 
we did not observe this significant difference in the game task, we believe it arises from the nature of the image docking task. In this task, users had to perform several small movements to put an image inside the frame and in this task there was a high possibility that the frame appear at the center of the screen and overlap with the central circle designs' feedback, which could cause distraction. One user commented, "In the central circle design, the circle is located exactly in the middle of the display, occupying a large space; therefore, the user could not easily observe the content."

In the case of the arrows design, although the performance times were still increased with feedback, the difference was not significant in any cases. This might be due to the fact that the arrows occupied a smaller part of the display and were located at the corners; as a result they caused less distraction in comparison to the central circle.
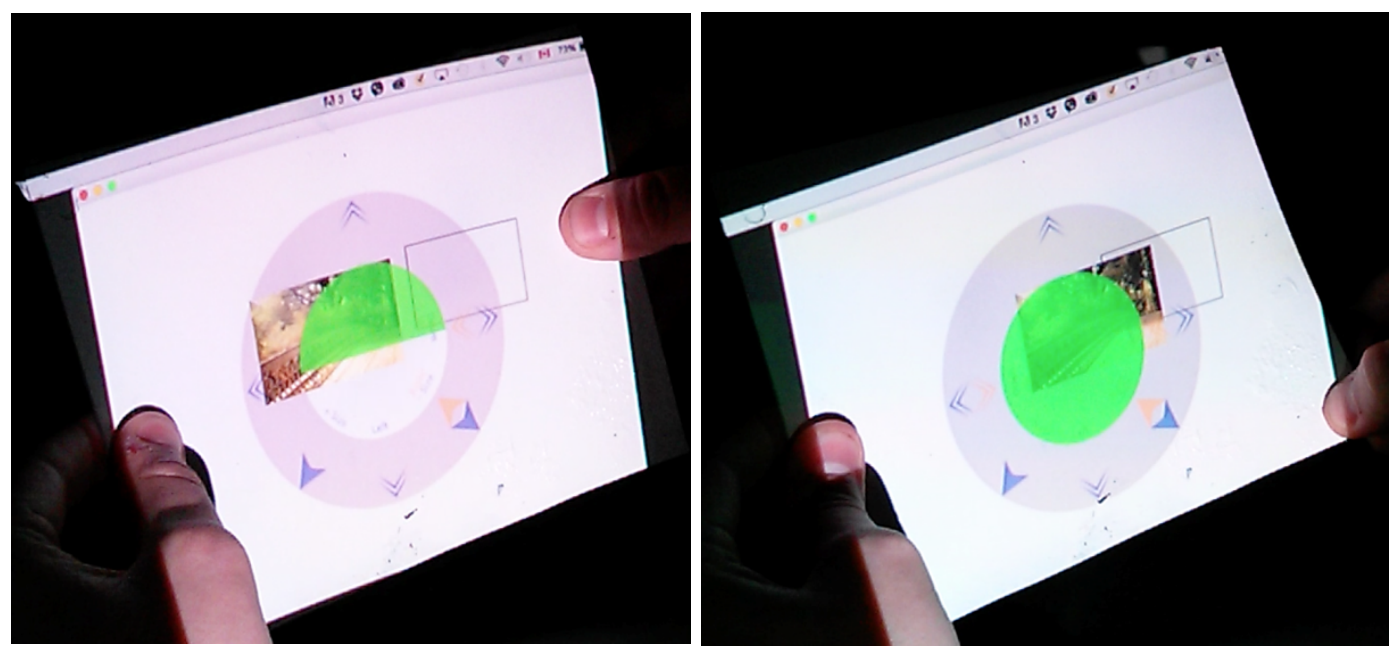

Figure 37: Central circle design covering the tasks' contents. 


\subsubsection{Feedback for wrong bends}

In addition to providing feedback about correctly performed bend gestures, we provided feedback when a user performed a wrong bend gesture. In the case of showing user errors during interactions with the prototype, we again observed no significant difference in the number of errors committed in the presence or absence of feedback. These results suggest that users did not require feedback to recognize their mistakes. One user declared, "I needed no feedback for understanding that I had made a mistake. When I didn't see the desirable result in the interface, I looked more closely into the feedforward, then performed the bend correctly." The results themselves provided some sort of feedback for users.

Although having feedback for errors did not improve users' performance during our experiment, we asked users to choose which of the following types of feedback would be best to receive on wrongly performed bend gestures: (1) arrows feedback (a red arrow at the location of the wrongly performed bend gesture); (2) central circle feedback ("X" at the middle of the screen); or (3) no feedback. Only $12 \%$ of participants chose no feedback, which means that users felt better when they had some kind of feedback and knew whether they were making mistakes. Another interesting point about feedback for wrong bend gestures was that the central circle design was the most preferred, according to the questionnaires. In some cases, the same users that performed better with the arrows design, preferred the central circle feedback design for wrong gestures. One explanation for this difference might be that, in the case of a wrongly performed bend gesture, the only thing that users needed was a small hint to warn them to stop. This might be 
because, when users want to perform a bend gesture, they should look at the actual location on the screen where they want to perform a bend gesture. However, when they start the bend gesture, users want to see the result of their action onscreen, but not necessarily at the location of the bend gesture. For instance, on a smartphone, when users try to adjust the volume of their smartphone, they look at the buttons, but once they start pressing the button, they look at the volume indicator on the phone.

We must note that one limitation of our study is that the feedback for correct bend gestures was always coupled with wrong bend gestures. If we had two conditions, where in one condition, the feedback only indicated correct bend gestures, and in the second condition, feedback only indicated wrong bend gestures, it might have resulted in a different outcome.

\subsection{Design Recommendations}

We propose the following design recommendations gathered from our experiment to help design visual feedback for flexible displays:

1. Showing the information at the actual location of the bend gesture is the best approach to take in handheld flexible displays. Not only did this method prove to be better in our quantitative data, but users clearly preferred this method.

2. Having feedback showing the angle of bend gestures is not necessary, and can lower performance. Our results showed that having feedback for bend gestures increased the time it took for tasks to be completed: some user reported it to be a distraction. 


\section{When showing the information at the location of the bend gesture,}

shapes should move from under the user's thumbs to make the shapes more visible (Figure 38). During our study we observed that the shapes and text became nonviewable for two reasons: 1 . The user's hand 2. Curve on the bend location. We propose for them to move slightly to avoid being occluded.

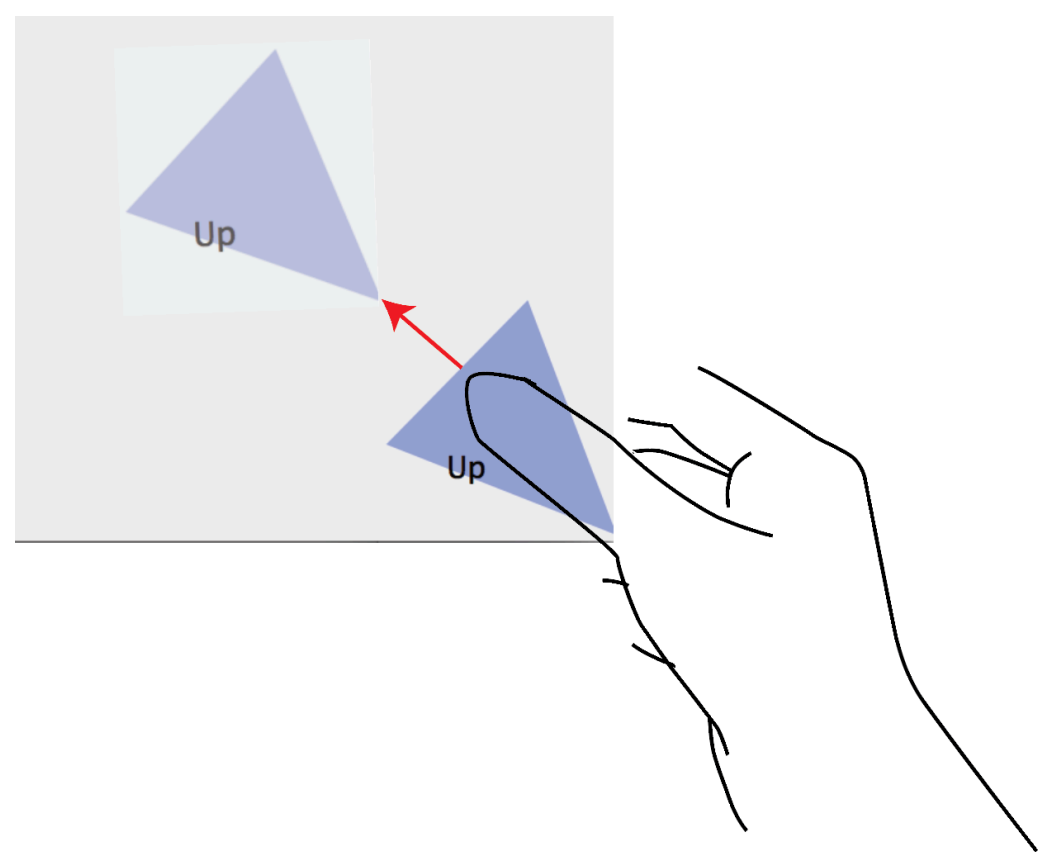

Figure 38: moving the shapes from under the user's thumbs

\section{Providing a small hint at the center of the display to indicate the} wrong bend gestures is a better way to provide feedback for wrong bend gestures. Between our two designs for showing wrong bend gestures, users preferred the one that had a "X," which provided a abstract hint about a wrong bend gesture. In general, in a flexible display, in which all the corners can be bent, the center is the only location that is always visible. As a result, the best location for showing the abstract information, which does not take up a large amount of the 
screen, is the center of the screen.

5. Visual feedback designs should contain a minimal amount of text. In our experiment, the cheat sheet design contained only text, and this amount of text was not good for most of our users. Specifically, directional words such as "vertical" and "horizontal" caused a lot of problems for some of the participants. By using different visual variables such as (1) location, (2) size, (3) shape, (4) orientation, (5) color value, (6) color saturation and (7) transparency [22], designers can remove these burdens of interpretation.

\section{Visual feedback designs should not occupy a large amount of the}

display. During our experiment, one of the drawbacks of the central circle design was its large size.

\subsection{Limitation}

The main limitation of this work is the physical engineering of the prototype display. We used Flexpoint bend sensors, which were not perfectly responsive to the small bend gestures that users made, and this had a negative effect on recognition process of the program. Additionally, the location of the horizontal sensor on the back of our prototype was not exactly at the center of the device and this could generate some problems on registration of the vertical bend. We conducted our user study with a mock-up display by using projection instead of a real flexible display. Though we tried to keep the position of the display on the prototype, we were unable to reduce distortion during bend interactions and this could have a negative effect on participants' speed of performance. We connected the prototype 
to a computer via a cable located on the bottom of the display. This became a source of some restrictions for participants' hand movements during the experimental sessions, which might have contributed to slowing the participants' movements. In addition, we evaluated our designs with simple tasks. However, everyday usage of cellphones and tablets might involve more practical and complicated tasks, such as typing or browsing the Internet. Therefore, it would be worth testing our design on such practical tasks.

Finally, The participatory design session's results were limited. The size of the group was limited (five), and the absence of expertise of the participants in the flexible displays area lead to ideas that were simple and lacked depth. It would be better to gather a group of people with skills in flexible displays or human-computer interaction. 


\section{Conclusion}

In this thesis, we presented a study on providing visual feedback for performing bend gestures on flexible displays. Our main goal in this study was to help users perform bend gestures correctly on a flexible device that offers bend gestures as its interaction style. We intended to educate users about the correct location, direction and angle of each available bend gesture. We also wanted to guide users in case of performing any wrong bed gestures to correct their mistake.

We began our exploration by conducting a participatory design session where users were asked to present novel ideas about designing such visual feedback. After analyzing the results of the participatory session, we developed three visual feedback designs using feedback and feedforward mechanisms: 1. central circle design, 2. arrows design, and 3. cheat sheet design. We conducted an experiment with two tasks to examine the effect of our visual feedback designs on users' performance while working with our flexible prototype. Our result showed that our two main designs (central circle design and arrows design) had a positive effect on users' performance, and they could improve users' speed of interaction. We observed that the feedback mechanism did not have any positive effect on users' performance. In addition, there was strong agreement among participants that: 1 . Showing the visual feedback information at the location of the actual bend made performing bend gestures easier, and 2. Feedback mechanisms were not helpful during their interaction with our flexible prototype. 


\subsection{Future work}

It would be worthwhile to investigate other feedback mechanisms, such as audio or tactile feedback. Adding audio feedback to our designs could guide users' perform bend gestures, by using different volumes to indicating the angle of a bend gesture. Similarly, a research on use of different frequency of vibration for indicating angle or size of the bend gestures will be useful. It would also be valuable to determine how much a visual feedback design that has the ability to increase users' interaction speed and reduce the error rates effectively reduce participants' fatigue in applications that are time-consuming and require a huge amount of bend gestures, such as complex games.

It would be valuable to test our designs on bigger or smaller screens to see the effect of a screen's size on the visual feedbacks.

It would also be interesting to measure the effect of such visual feedback design on the age of the materials of a device. When users perform bend gestures with less unnecessary pressure or fewer wrong bend gestures, the result could be that hardware of the device will be healthier. Finally, it might be valuable to test the visual feedback designs on screens with different sizes also considering other factors of a bend gesture such as speed of a bend gesture or size of a bend will also have valuable benefits. 


\section{Appendices}

Appendix A: Questionnaires

A.1 Pre- Test Questionnaire

\section{Visual feedback for flexible displays}

There are 7 questions in this survey

\section{Demographic information}

\section{[]ID: *}

Please write your answer here:

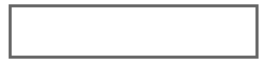

[]Age: *

Please write your answer here:

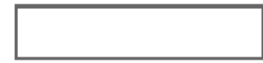

[]Handedness *

Please choose only one of the following:

Right

Left

Ambidextrous

[]Gender: *

Please choose only one of the following:

Male

Female 
[]Have you ever used a flexible (deformable) device? *

Please choose only one of the following:

Y Yes

No

Make a comment on your choice here:

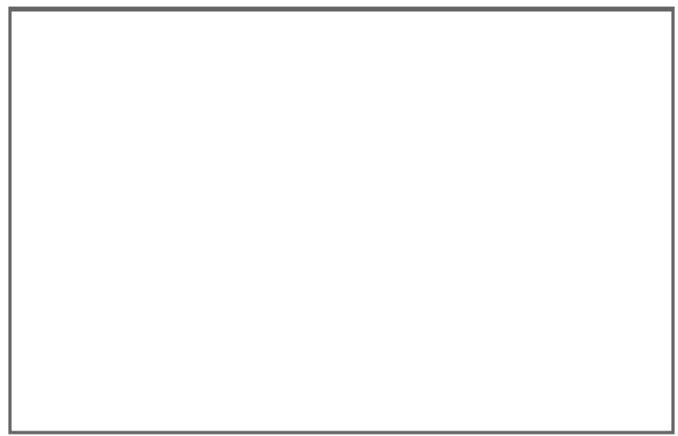

If yes, plesae explain

[]Do you have a smartphone? *

Please choose only one of the following

Yes

No

[]

If you have a smartphone how often do you use it?: *

Please choose only one of the following:

All the time

Quite often

Regularly

Once in a while

Rarely

http://cil.csit.carleton.ca/s/index.php/admin/printablesurvey/sa/index/surveyid/281418

Page 2 of 3 


\section{A.2 Experiment Questionnaire}

\section{Form \# 2}

\section{[]ID\# *}

Please write your answer here:

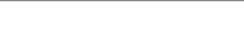

[]Task- Interface *

Please write your answer here: 
[]Please indicate how much you agree with the following statements: *

Please choose the appropriate response for each item:

\begin{tabular}{|c|c|c|}
\hline $\begin{array}{l}\text { strongly } \\
\text { agree }\end{array}$ & $\begin{array}{c}\text { somewhat } \\
\text { agree }\end{array}$ & neutral \\
\hline
\end{tabular}

This design guides

the user to easily find

the location of the

available bends.

This design guides

the user to easily find

the direction of each

bend (upward or

downward).

This design does not distract the user from

seeing the actual

content of the

application.

This design indicates

clearly when a wrong

bend is performed.

This design indicates

clearly when a

gesture requires two

locations to bend at a

0

0

$\mathrm{O}$

same time.

The half activation

indicator was useful

during performing a

bend.

0

0

0

O

$0 \quad 0$

0

$\mathrm{O}$

$\mathrm{O}$

$0 \quad 0$

$0 \quad 0$

0

O

0

0

0

$0 \quad 0$

0

0

0

[]On a scale of 1 to 5 , how useful was the green activation indicator during performing a bend.

Please choose only one of the following:

O 1

O 2

3

O 4

5

$1=$ not useful at all , $5=$ Very useful 


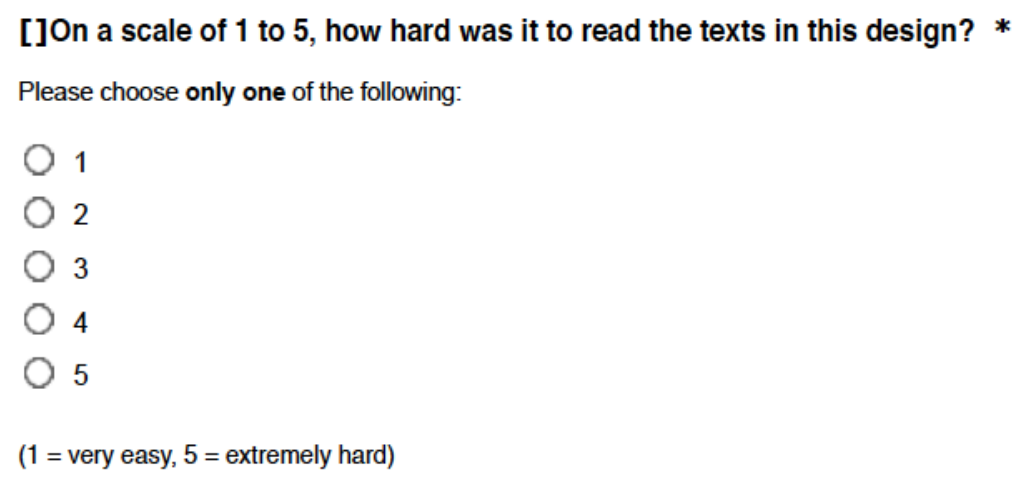


[]What did you find most frustrating about this design? *

Please write your answer here:

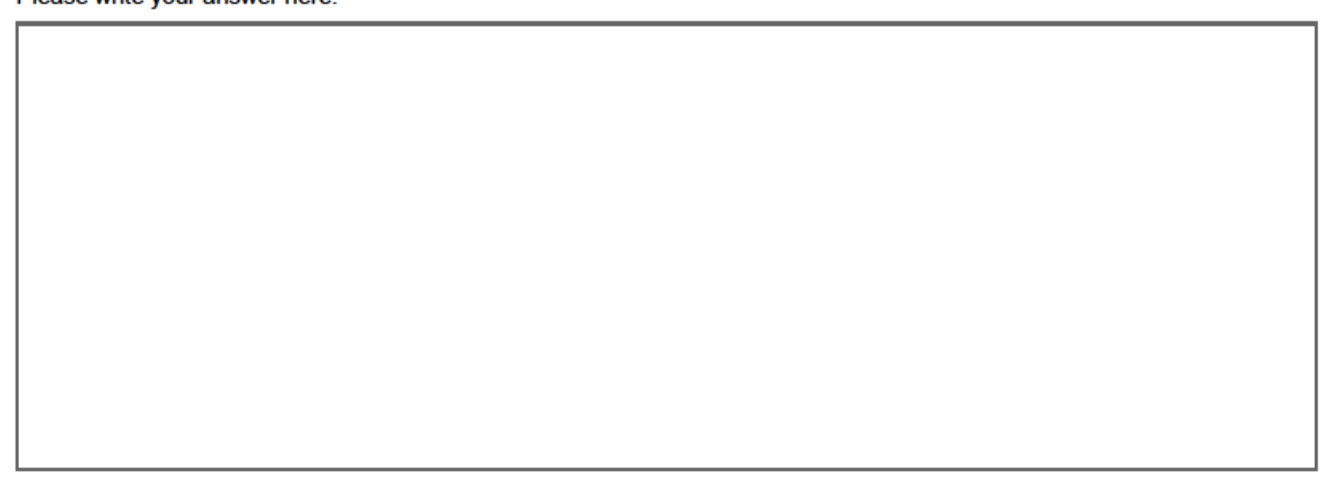

[]lf you could change one thing about this design, what would it be and why? *

Please write your answer here: 


\section{A.3 Final Questionnaire}

\section{Form \#3}

\section{[]ID\# *}

Please write your answer here:

[]From 1 to 3 , please rank the designs according to your preference. *

Please number each box in order of preference from 1 to 3

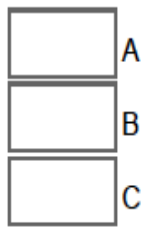

(1. Most preferred, 3. Least preferred)

A.

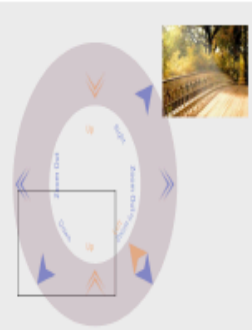

B.

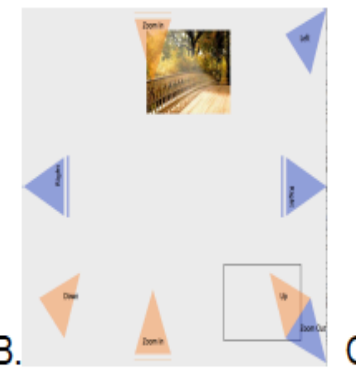

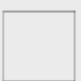

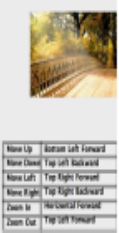


[]From 1 to 3 , please rank the designs in terms of their visual appeal *

Please number each box in order of preference from 1 to 3

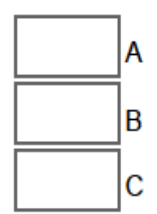

( 1 = no appeal, $3=$ very appealing).

A.
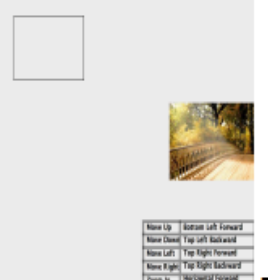

B.

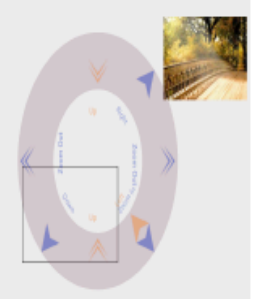

C.

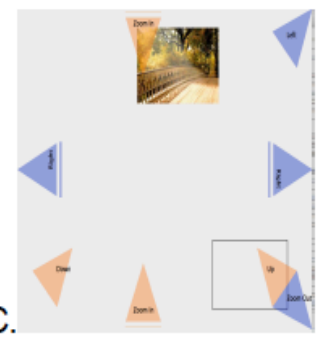

[]From 1 to 3 , please rank the designs according to how much useful information they include.

Please number each box in order of preference from 1 to 3

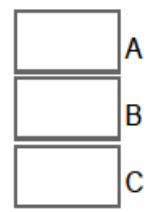

1. Uninformative, 2. Somewhat informative, 3. Very informative
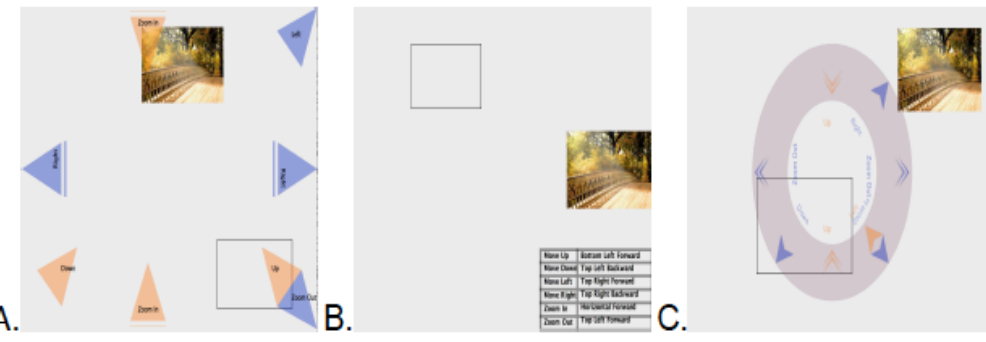
[]Overall, which one of the following feedback designs did you like the most(Why?): *

Please choose only one of the following:

$\mathrm{OA}$

$\mathrm{B}$

No Feedback

Make a comment on your choice here:
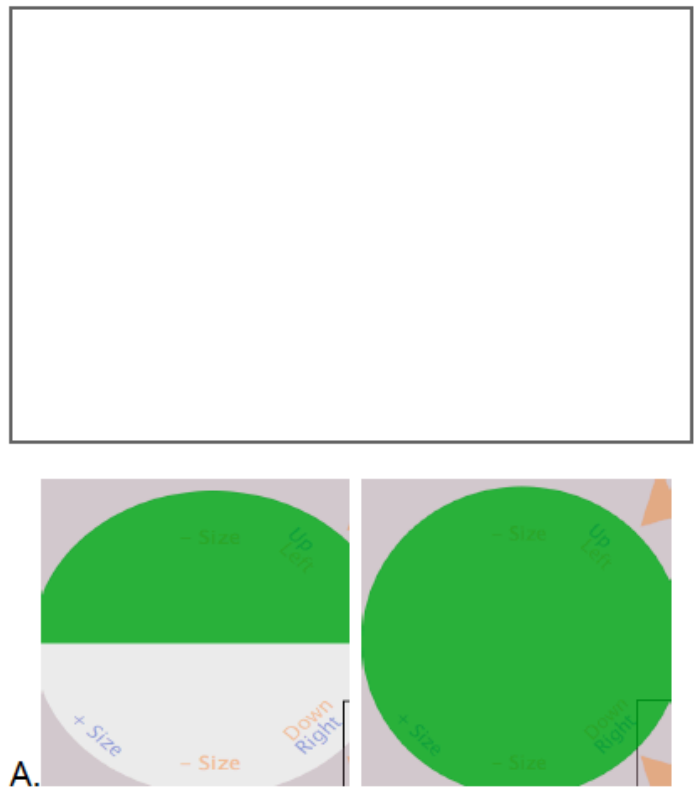

B.
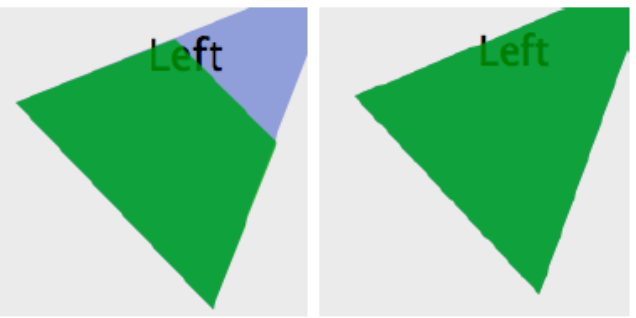
[]Overall, which one of the following feedback designs for errors did you like the most (Why?): *

Please choose only one of the following:

$\mathrm{OA}$

$\mathrm{OB}$

No Feedback

Make a comment on your choice here:

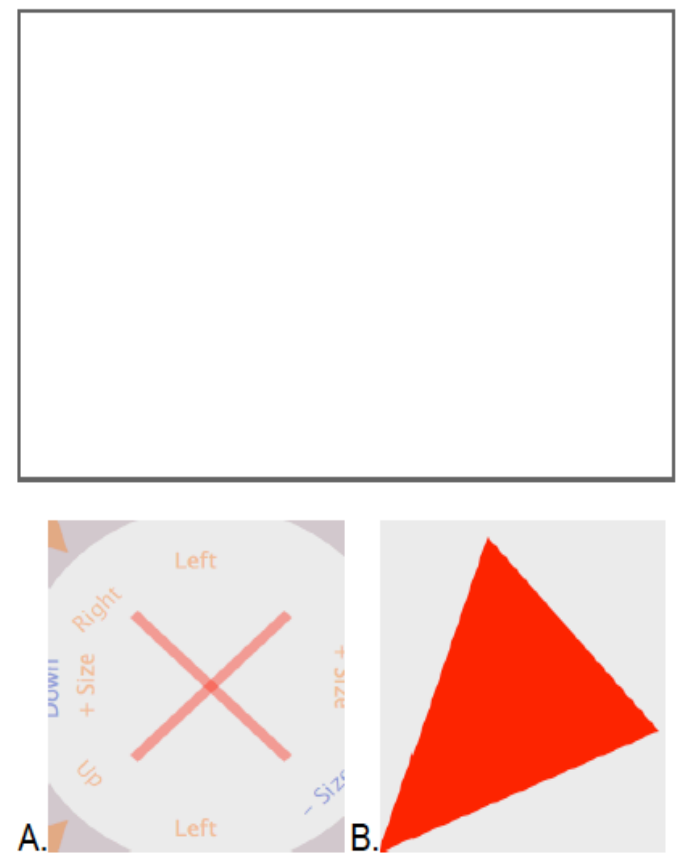

[]Overall, using visual feedback was more useful in performing...... *

Please choose only one of the following:

Task 1(Image Docking)

Task2(The Game)

Both equally 
[]Any final comments? *

Please write your answer here: 


\section{References}

[1] Ahmaniemi, T., Kildal, J., and Haveri, M.What is a device bend gesture really good for? Proceedings of the 32nd annual ACM conference on Human factors in computing systems, (2014), 3503-3512.

[2] Armand, M. and Tarascon, J.-M.Building better batteries. Nature 451, 7179 (2008), 652-657.

[3] Balakrishnan, R. and Fitzmaurice, G.Exploring interactive curve and surface manipulation using a bend and twist sensitive input strip. In Proceedings of the 1999 symposium on Interactive 3D graphics, (1999), 111-119.

[4] Bau, O. and Mackay, W.E.OctoPocus: a dynamic guide for learning gesturebased command sets. Proc. UIST, (2008), 37-46.

[5] Curtis, S.Quarter of the world will be using smartphones in 2016. http://www.telegraph.co.uk/technology/mobile-phones/11287659/Quarterof-the-world-will-be-using-smartphones-in-2016.html.

[6] Djajadiningrat, T., Overbeeke, K., and Wensveen, S.tell us how ? through feedforward and inherent feedback. In Proceedings of the 4th ACM conference on Designing interactive systems: processes, practices, methods, and techniques, (2002), 285-291.

[7] Freeman, D., Benko, H., Morris, M.R., and Wigdor, D.ShadowGuides: Visualisations for In-Situ Learning of Multi-Touch and Whole-Hand Gestures. Proceedings of the ACM International Conference on Interactive Tabletops and Surfaces - ITS '09, (2009), 165-172.

[8] Gallant, D., Seniuk, A., and Vertegaal, R.Towards more paper-like input: flexible input devices for foldable interaction styles. Proceedings of the 21st annual ACM symposium on User interface software and technology, (2008), 283-286.

[9] Girouard, A., Lo, J., Riyadh, M., Daliri, F., Eady, A.K., and Pasquero, J.OneHanded Bend Interactions with Deformable Smartphones. Proceedings of the 33rd Annual ACM Conference on Human Factors in Computing Systems - CHI '15, ACM Press (2015), 1509-1518.

[10] Gomes, A., Nesbitt, A., and Vertegaal, R.MorePhone: A Study of Actuated Shape Deformations for Flexible Thin-Film Smartphone Notifications. Proceedings of the SIGCHI Conference on Human Factors in Computing Systems - CHI '13, (2013), 583-592. 
[11] Guimbretiere, F. and Winograd, T.FlowMenu : Combining Command, Text, and Data Entry. ACM Symposium on User Interface Software and Technology, (2000), 213-216.

[12] Herkenrath, G., Karrer, T., and Borchers, J.Twend: twisting and bending as new interaction gesture in mobile devices. CHI'08 Extended Abstracts on Human Factors in Computing Systems, (2008), 3819-3824.

[13] Holman, D., Vertegaal, R., Altosaar, M., Troje, N., and Johns, D.PaperWindows : Interaction Techniques for Digital Paper. Proc. CHI, (2005), 591-599.

[14] Kildal, J., Paasovaara, S., and Aaltonen, V.Kinetic Device : Designing Interactions with a Deformable Mobile Interface. Proc. CHI EA, (2012), 18711876.

[15] Kildal, Lucero, and Boberg, M.Twisting touch: combining deformation and touch as input within the same interaction cycle on handheld devices. Proceedings of MobileHCI 2013, (2013), 237-246.

[16] Konieczny, J., Shimizu, C., Meyer, G., and Colucci, D.A handheld flexible display system. Visualization, 2005., (2005), 591-597.

[17] Kurtenbach, G. and Buxton, W.The limits of expert performance using hierarchic marking menus. Proceedings of the INTERACT'93 and CHI'93 conference on Human factors in computing systems, (1993), 482-487.

[18] Kurtenbach, G., Sellen, A., and Buxton, W.An Empirical Evaluation of Some Articulatory and Cognitive Aspects of Marking Menus. Human-Computer Interaction 8, 1993, 1-23.

[19] Lahey, B. and Girouard, A.PaperPhone: understanding the use of bend gestures in mobile devices with flexible electronic paper displays. Proceedings of the SIGCHI Conference on Human Factors in Computing Systems, (2011), 1303-1312.

[20] Lee, S., Lim, Y., and Lee, K.-P.Exploring the effects of size on deformable user interfaces. Proc. CHI, (2012), 89.

[21] Lee, S.-S., Kim, S., Jin, B., et al.How users manipulate deformable displays as input devices. Proceedings of the 28th international conference on Human factors in computing systems - CHI '10, (2010), 1647.

[22] Leflar, M. and Girouard, A.Navigating in 3D space with a handheld flexible device. Entertainment Computing 5, 4 (2014), 205-209. 
[23] Lo, J. and Girouard, A.Fabricating Bendy, Pervasive Computing IEEE (2014), $40-46$.

[24] Lo, J.Bendy: An Exploration into Gaming with Mobile Flexible Devices by Affairs in partial fulfillment of the requirements for the degree of Masters of Applied Sciences. (2013).

[25] Maqsood, S., Chiasson, S., and Girouard, A.POSTER: Passwords on flexible display devices. Proceedings of the 2013 ACM SIGSAC conference on Computer \& communications security - CCS '13, (2013), 1469-1472.

[26] Perlin, K.Quikwriting: continuous stylus-based text entry. Proceedings of the 11th annual ACM symposium on User interface software and technology, (1998), 3-4.

[27] St. Pierre, R.The UX book, process and guidelines for ensuring a quality user experience by Rex Hartson and Pardha S. Pyla. ACM SIGSOFT Software Engineering Notes 37, 2012, 43.

[28] Schwesig, C., Poupyrev, I., and Mori, E.Gummi: A Bendable Computer. Proc. CHI, (2004), 263-270.

[29] Shneiderman, B.Designing the User Interface. 1998.

[30] Spinuzzi, C.The Methodology of Participatory Design. Technical Communication 52, 2 (2005), 163-174.

[31] Steimle, J., Jordt, A., and Maes, P.Flexpad: highly flexible bending interactions for projected handheld displays. Proceedings of the SIGCHI Conference on Human Factors in Computing Systems - CHI'13, (2013), 237-246.

[32] Tajika, T., Yonezawa, T., and Mitsunaga, N.Intuitive page-turning interface of e-books on flexible e-paper based on user studies. Proceeding of the 16th ACM international conference on Multimedia - MM '08, (2008), 793.

[33] Vanacken, D., Demeure, A., Luyten, K., and Coninx, K.Ghosts in the interface: Meta-user interface visualizations as guides for multi-touch interaction. 2008 IEEE International Workshop on Horizontal Interactive Human Computer System, TABLETOP 2008, (2008), 81-84.

[34] Vermeulen, J., Luyten, K., Hoven, E. Van Den, and Coninx, K.Crossing the Bridge over Norman 's Gulf of Execution : Revealing Feedforward 's True Identity. Proc. CHI, (2013), 1931-1940.

[35] Warren, K., Lo, J., Vadgama, V., and Girouard, A.Bending the Rules: Bend Gesture Classification for Flexible Displays. Proc. CHI, (2013). 
[36] Watanabe, J., Mochizuki, A., and Horry, Y.Bookisheet: Bendable Device for Browsing Content Using the Metaphor of Leafing Through the Pages. Proc. UbiComp, (2008), 360-369.

[37] Wigdor, D., Williams, S., and Cronin, M.Ripples: utilizing per-contact visualizations to improve user interaction with touch displays. In Proceedings of the 22nd annual ACM symposium on User interface software and technology, (2009).

[38] Wightman, D., Ginn, T., and Vertegaal, R.BendFlip: Examining Input Techniques for Electronic Book Readers with Flexible Form Factors. Proc. INTERACT, Springer Berlin / Heidelberg (2011), 117-133.

[39] Ye, Z. and Khalid, H.Cobra: Flexible Displays for Mobile Gaming Scenarios. Proc. CHI EA, (2010), 4363-4367.

[40] Wicked Problems: Problems Worth Solving - Participatory Design. https://www.wickedproblems.com/4_participatory_design.php.

[41] Flexpoint Sensor Systems. http://www.flexpoint.com/. 